University of Rhode Island

DigitalCommons@URI

Open Access Dissertations

2018

\title{
A HYBRID LATTICE BOLTZMANN METHOD-POTENTIAL FLOW MODEL FOR NAVAL HYDRODYNAMICS
}

Christopher M. O'Reilly

University of Rhode Island, chrisoreilly123@cox.net

Follow this and additional works at: https://digitalcommons.uri.edu/oa_diss

\section{Recommended Citation}

O'Reilly, Christopher M., "A HYBRID LATTICE BOLTZMANN METHOD-POTENTIAL FLOW MODEL FOR NAVAL HYDRODYNAMICS" (2018). Open Access Dissertations. Paper 807.

https://digitalcommons.uri.edu/oa_diss/807

This Dissertation is brought to you for free and open access by DigitalCommons@URI. It has been accepted for inclusion in Open Access Dissertations by an authorized administrator of DigitalCommons@URI. For more information, please contact digitalcommons-group@uri.edu. 
A HYBRID LATTICE BOLTZMANN METHOD-POTENTIAL FLOW MODEL FOR NAVAL HYDRODYNAMICS

BY

CHRISTOPHER M. O'REILLY

A DISSERTATION SUBMITTED IN PARTIAL FULFILLMENT OF THE

REQUIREMENTS FOR THE DEGREE OF

DOCTOR OF PHILOSOPHY

IN

OCEAN ENGINEERING

UNIVERSITY OF RHODE ISLAND

2018 


\section{DOCTOR OF PHILOSOPHY DISSERTATION}

$\mathrm{OF}$

CHRISTOPHER M. O'REILLY

\section{APPROVED:}

Dissertation Committee:

$\begin{array}{ll}\text { Major Professor } & \text { Stephan T. Grilli } \\ & \text { Jason M. Dahl } \\ & \text { M. Reza Hashemi } \\ & \text { Tetsu Hara } \\ & \text { Nasser H. Zawia } \\ & \text { DEAN OF THE GRADUATE SCHOOL }\end{array}$

UNIVERSITY OF RHODE ISLAND 


\begin{abstract}
The simulation of many naval hydrodynamics problems, such as a ship's motions in waves, is often performed using potential flow solvers which are usually based on a Boundary Element Method (BEM) that use semi-empirical corrections to account for viscous/turbulent effects. However in some cases, viscous/turbulent flows near the ships hull and breaking waves must be accurately modeled to capture the salient physics. Navier-Stokes (NS) solvers can and have been used to model such flows, but they are computationally expensive, often requiring several orders of magnitude more computational resources relative to potential flow methods, rendering them impracticable for many engineering applications. The overall goal of this work is to develop a naval hydrodynamics solver that leverages a medium-fidelity potential flow solver to model the entire domain combined with a high-fidelity Navier-Stokes (NS) solver to model the flow within a smaller region, where better accuracy is required. This hybrid solver provides improved simulation fidelity relative to a potential flow solution alone while a significant computational efficiency improvement relative to a NS solver alone is gained.

Within the NS domain both the velocity and pressure are expressed as the sum of an inviscid (I) and viscous perturbation $(\mathrm{P})$ components. The underlying inviscid solution serves to drive the perturbation component, which in turn provides a correction so that the total solution reproduces the NS equations. Considering that most naval hydrodynamics flows occur at high Renyolds numbers, the viscous region of the flow is often small, and can be applied to a reduced domain around a hull or to localized regions within the flow. Outside of these regions the salient viscous effects will become small and the inviscid solver provides the full NS solution.

In this work the NS domain is simulated using the particle based Lattice Boltzmann Method (LBM). This relatively new computational tool has proved to be accurate and efficient for simulating a variety of complex fluid flow and fluid-structure interaction problems. It shows the potential for a competitive advantage over traditional finite volume NS solvers when implemented in parallel on Graphics Processing Units (GPUs). The LBM is well suited for the GPU architecture because its kernel is simple and local, so at each time step relatively small number of operations is required at each node and nodes only communicate with their neighbors. This is opposed to finite volume solvers which typically require high order derivatives and a global pressure correction step, where all nodes need to communicate and more complex memory access is required. Using the LBM our hybrid method can make efficient use of a computer's resources by simulating the
\end{abstract}


BEM using the central processing unit (CPU) nodes and simulating the LBM using a relatively inexpensive GPU addition, allowing for simulations that would otherwise require a large and expensive CPU cluster.

The goal of this thesis is to develop a LBM that can solve for the perturbation component of our hybrid method, which requires a modification to the LBMs governing equations, boundary, and initial conditions. The first chapter describes the fundamental developments towards this goal of developing what we refer to as a perturbation LBM (pLBM) and presents several low Reynolds number validations of the method's accuracy and convergence. The second chapter focuses on higher Reynolds number applications of the method. Since the LBM is far less established than other methods, this required that we develop an accurate turbulent wall boundary condition for standard LBM, which is currently an active area of research in the LBM community. Next the turbulent wall model and a large eddy simulation (LES) turbulent closure schemes are expressed for the pLBM by using the standard LBM methodology as a foundation and a validated for turbulent applications is presented. The third chapter focuses on the hybrid modeling of the nonlinear free surface and adapting the pLBM tool to simulate ship geometries. A hybrid volume of fluid (hVOF) free surface capturing scheme is developed which models the total free surface using a combination of the inviscid and perturbation flow within the pLBM. Finally, pLBM is coupled to a BEM solver to simulate the steady flow around a ship and the hVOF is used to simulate nonlinear and breaking waves. 


\section{ACKNOWLEDGMENTS}

"Always remember to love the questions and live the answers."

- Rev. Dom Ambrose Wolverton

I would like to express my gratitude to the impressive and kind people who have guided me through this academic journey. First, I would like to thank professors Stephan Grilli and Jason Dahl. Stephan has been a role model to me since my undergraduate years. He has provided remarkable examples of academic excellence, work ethic, and generosity that I strive to emulate. Ever since teaching me basic hydrodynamics Jason has consistently found a way to explain complicated concepts to me in terms that I understand and he has always taken the time to help. I would like to thank Dave Kring for providing me with the dream opportunity to finish my degree while working at Navatek. Dave has become a mentor and role model to me and I admire him greatly. I will always be grateful for what professor Anette Grilli has done for me. During my master's degree she showed me that I was capable of more than I had realized and she inspired me to pursue a doctorate. Thank you to Christian Janßen, who graciously spent many hours teaching me about the lattice Boltzmann method and has turned into both a colleague and a friend. Thank you to Dominic Piro, who I have had a lot of fun learning from. Finally, thank you to the many friends who have helped me through this process by providing the perspective and support that I needed. 


\section{DEDICATION}

To my parents and family. I am profoundly fortunate to have such loving and supportive people in my life. 


\section{PREFACE}

This thesis follows the manuscript format and consists of 3 papers. The first, titled "A lattice-Boltzmann-based perturbation method" will be submitted for review to the Journal of Computers and fluids. The second, titled "A hybrid lattice-Boltzmann method and potential flow solver applied to high Reynolds number incompressible flows" will be submitted for review to the Journal of Computational Physics. Finally the third manuscript, titled "Free surface modeling using a hybrid lattice-Boltzmann and potential flow model", will be submitted for publication in the future. 


\section{Contents}

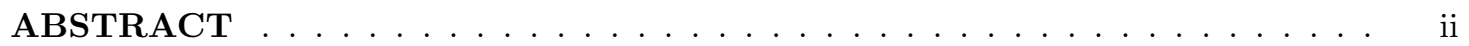

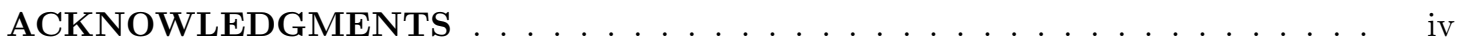

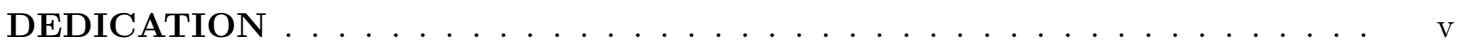

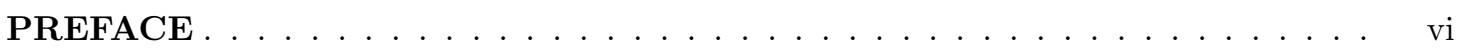

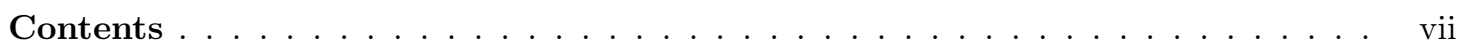

List of Figures . . . . . . . . . . . . . . . . . . . . . . . . .

List of Tables ................................ xiv

\section{Chapter}

1 A lattice-Boltzmann-based perturbation method . . . . . . . . . 1

1.1 Introduction . . . . . . . . . . . . . . . . . . . 2

1.2 Lattice Boltzmann Method $(\mathrm{LBM}) \ldots \ldots \ldots \ldots$

1.2 .1 LBM fundamentals $\ldots \ldots \ldots \ldots \ldots \ldots \ldots$

1.2 .2 Collision operators $\ldots \ldots \ldots \ldots \ldots$

1.3 Perturbation Lattice Boltzmann Method $(\mathrm{pLBM}) \ldots \ldots \ldots$

1.3.1 Coupling approach through the hybrid NS equations . . . . . . . . 9

1.3.2 Derivation of pLBM collision operators . . . . . . . . . . . 10

1.3.3 Recovering the perturbation NS equations from the pLBM . . . . . . 12

1.3.4 Implementation of the $\mathrm{pLBM} \ldots \ldots \ldots \ldots$

1.4 Applications . . . . . . . . . . . . . . . . . . . . . . . . . 17

1.4.1 Oscillatory boundary layers over a plane solid wall . . . . . . . . . 17

1.4.2 Viscous flow past a circular cylinder . . . . . . . . . . . . . 22

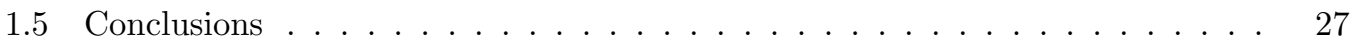

1.6 Acknowledgments . . . . . . . . . . . . . . . . . . . . . 27

2 A hybrid lattice-Boltzmann method and potential flow solver applied to high Reynolds number incompressible flows . . . . . . . . . . . . . . 34 
2.1 Introduction . . . . . . . . . . . . . . . . . . . . . 35

2.2 The Lattice Boltzmann Method . . . . . . . . . . . . . . . . . . . . . . 38

$2.2 .1 \quad$ LBM basics . . . . . . . . . . . . . . . . . . . . . . . . . 39

2.2 .2 Equations for the perturbation LBM . . . . . . . . . . . . . . . 41

2.2.3 LES turbulence modeling with the perturbation LBM $\ldots \ldots$. . . . . 42

2.3 Turbulent Wall Model . . . . . . . . . . . . . . . . . . . . . . . . . . . . . 43

2.4 Applications . . . . . . . . . . . . . . . . . . . . . . . 47

2.4.1 Simulation of turbulent flow over a flat plate . . . . . . . . . . 47

2.4 .2 Turbulent Foil Simulation . . . . . . . . . . . . . . . . . 50

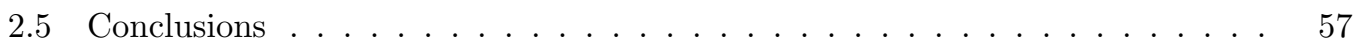

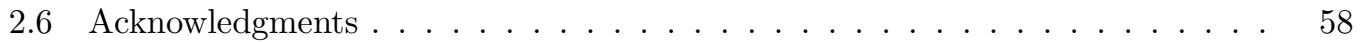

2.7 Appendix A: Recovering the perturbation NS equations from the perturbation LBM . . . . . . . . . . . . . . . . . . . . . 58

3 Free surface modeling using a hybrid lattice-Boltzmann and potential flow model . . . . . . . . . . . . . . . . . . . . . . . . . . . . 68

3.1 Introduction . . . . . . . . . . . . . . . . . . . . 69

3.2 Lattice Boltzmann Method $(\mathrm{LBM}) \ldots \ldots \ldots$. . . . . . . . . . 72

$3.2 .1 \quad$ LBM fundamentals . . . . . . . . . . . . . . . . . . . 73

3.2 .2 Collision operators . . . . . . . . . . . . . . . . . . . 74

3.2 .3 Equations for the perturbation $\mathrm{LBM} \ldots \ldots \ldots 76$

3.3 Free Surface Boundary Conditions with the Perturbation Method . . . . . . . 77

3.4 Hybrid Volume of Fluid Method _ . . . . . . . . . . . . . . . . . . 79

3.4.1 Dynamic Boundary Condition with the pLBM . . . . . . . . . 80

3.4 .2 Initialization of New Interface Nodes . . . . . . . . . . . . . . 81

3.4.3 The Perturbation NS and Total NS interface . . . . . . . . . 82

3.5 Power Dissipation Due to Wave Breaking . . . . . . . . . . . . . . . . 82

3.6 Gridding General Geometries in LBM with the Turbulent Wall Model . . . . 83

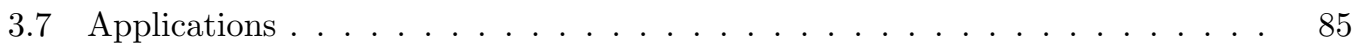

3.7.1 Coupling the perturbation LBM to a BEM solver . . . . . . . . . 85 
3.7 .2 Nonlinear free surface modeling . . . . . . . . . . . . . . . . . 89

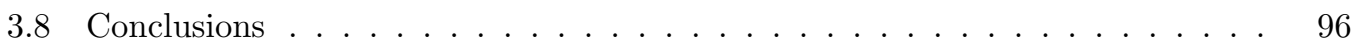

3.9 Acknowledgments . . . . . . . . . . . . . . . . . . . . . . . . 97 


\section{List of Figures}

1 (a) Domain setup for the Stokes OLBL simulation. The far-field periodic potential flow velocity is specified in the Inviscid domain, while the pLBM model is applied within the Hybrid domain. (b) Parameters used in numerical model applications . . . . . . . . . . . . . . . . . . . .

2 (a) Analytical horizontal velocity profile $u(z, t)$ within an OLBL [50], from Eq. (49); each line corresponds to a different phase angles $\Phi=\omega t$ (see Fig. 1 for physical parameters). (b) Maximum numerical error of the pLBM or LBM OLBL solution as a function of the Reynolds number $R e_{\delta}$ and the discretization $N_{z}$; note the quadratic convergence rate with $1 / N_{z} . \ldots . . . .$.

3 (a) Domain setup for the steady streaming OLWBL simulation. The far-field periodic potential flow velocity is specified in the Inviscid domain, while the pLBM model is applied within the Hybrid domain. (b) Parameters used in numerical model applications . . . . . . . . . . . . . . . .

4 Normalized steady streaming velocity profile (i.e., period-averaged) $u^{*}=$ $\bar{u}_{1}\left(\frac{A^{2} \omega k}{\sinh ^{2} k h}\right)^{-1}$ in the OLWBL, for 4 different pLBM grids with vertical resolution $N_{z}=\delta_{s} / \Delta x=26(\bullet), 51(\bullet), 101(\bullet), 201(\bullet)$ and Mach Number Ma (as indicated), compared to the analytical solution given by Eq. (56). . . . . . .

Convergence of numerical errors of the normalized period-averaged steady streaming velocity $u^{*}$, in the results of Fig. 4, in terms of the $L_{1}$ (Eq. (53)) norms of the relative errors. Results shown are for successively increasing spatial and time resolutions as $\left(N_{z}, \mathrm{Ma}\right)=(26,0.08),(51,0.04),(101,0.02)$, and

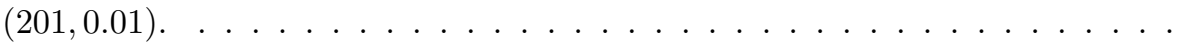

$6 \quad$ Nested domain set-up for the LBM and pLBM simulations of the viscous flow past a circular cylinder of diameter $D$, and associated geometric parameters for each grid; the expansion ratio indicates how the mesh size $\Delta x$ changes from one grid to the other. The cylinder center is located at $(0,0)$. . . . . . .

$7 \quad$ Instantaneous velocity magnitude normalized by the flow free-stream velocity $U$ around a cylinder of diameter $D$, at $\operatorname{Re}=100$ during steady state vortex shedding. Total velocity calculated with the LBM (a) and hybrid pLBM (b) are compared at a similar phase of the steady state vortex shedding cycle. The Inviscid velocity $u_{i}^{I}$ driving the hybrid LBM solution (c) and perturbation velocity $u_{i}^{P}(\mathrm{~d})$ are shown, representing the decomposed components of the

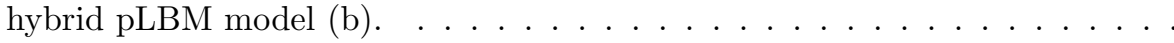

8 Sketch of LBM flow reconstruction near a solid boundary (assumed 2D for simplicity). Boundary layer profile quantities and regions are described on the left, while LBM boundary node reconstruction is seen on the right. Known (- ) and missing (- - - ) DF populations are shown, along with subgrid wall distance $q$. This image is present in a wall coincident coordinate system of $x^{*}$ and $y^{*}$ based on unit normal $(\hat{n})$ and tangential vectors $(\hat{t})$ to the wall. . . . 
$9 \quad$ Mean velocity $u^{+}$as a function of distance $y^{+}$above the plate calculated using the hybrid LBM and turbulent wall model, at $\operatorname{Re}_{m}=37,042$ (a), 87,000 (b), and $1.21 \times 10^{6}(\mathrm{c})$. Numerical results (symbols) are plotted for a half channel of resolution $N=(\bullet) 10,(\square) 20,(\Delta) 30$, and $N=40(\checkmark)$, compared to the velocity profile of [Musker, 1979] (-). For visualization purposes, results for $N=20,30$, and 40 are shifted by $\Delta u^{+}=10,20$, and 30, respectively. (d) Friction coefficient $C_{f}$ computed, compared to [Dean, 1976] (-) and the upper and lower bounds of his measurements ( - - ) as a function of Reynolds number. . . . . . . . . . . . . . . . . . .

10 Turbulent fluctuation of the flow at $\operatorname{Re}_{m}=37,042$ (a) and 87,000 (b) calculated with the hybrid LBM with dots representing $u^{\prime+}(\bullet), v^{++}(\bullet), w^{\prime+}(\bullet)$ and $u^{\prime} v^{\prime+}(\bullet)$ as compared to the DNS values of [Hoyas and Jiménez, 2009] (shown as lines) as a function of wall distance $y$ and calculated using a resolution $N=4050$

11 Nested domain set-up for the LBM and pLBM simulations of a NACA0012 foil with its leading edge defined at $x=y=z=0.0$ and chord length $C$. The foil span extends beyond the cross stream extents (z-direction) of all meshes. (a) The associated geometric parameters for each grid; the expansion ratio indicates how the mesh size $\Delta x$ changes from one grid to the other. In the pLBM simulations Grid 0 is eliminated. (b) a visualization of the of the edges (black lines) of Grids 1-3 in reference to the foil seen in orange. . . . . . . . .

Lift (a) and drag (b) coefficient of a NACA0012 foil, as a function of its angle of attack. LBM simulation results, calculated at $\mathrm{Re}=1.44 \times 10^{6}$, are plotted as dots, for minimum Grid 3 resolution $(\Delta x / C)$ of: $4.0 \times 10^{-3}(\bullet), 3.5 \times$ $10^{-3}(\bullet), 3.0 \times 10^{-3}(\bullet), 2.5 \times 10^{-3}(\bullet)$. Xfoil simulation results are plotted as black diamonds $(\diamond)$, the measurements of [Gregory and OReilly, 1973] for Re $=1.44 \times 10^{6}$ for a rough foil $(-)$, and smooth foil $(---)$, and the measurements of [Sheldahl and Klimas, 2006] at $\operatorname{Re}=1.36 \times 10^{6}$ for a smooth foil $(---)$. .

13 Negative pressure coefficients $\left(C_{p}\right)$ plotted on the surface of the NACA0012 foil, calculated at $\operatorname{Re}=1.44 \times 10^{6}$ for $\theta=0^{\circ}(\mathrm{a})$ and $\theta=8^{\circ}(\mathrm{b})$. Xfoil results $(-)$ are compared to LBM results for $(\Delta x / C)=3.5 \times 10^{-3}(-), 3.0 \times 10^{-3}$

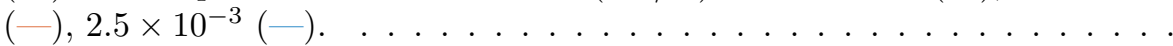

14 (a) Comparison of the Karman-Trefftz foil profile (-) used to compute the potentiaal flow solution with the NACA0012 foil profile (- -). (b) Visualization of the magnitude of velocity calculated with the pLBM for a steady state and $\theta=4^{\circ}$; with total velocity, $u_{i}^{T}=u_{i}^{I}+u_{i}^{P}$ (top), the inviscid velocity, $u_{i}^{I}$, (middle), and perturbation velocity, $u_{i}^{P}$ (bottom). . . . . . . . . .

15 Lift (top) and drag (bottom) coefficient of a NACA0012 foil, as a function of its angle of attack. pLBM simulation results, calculated at $\operatorname{Re}=1.44 \times 10^{6}$, are plotted as dots, for minimum Grid 3 resolution $(\Delta x / C)$ of: $4.0 \times 10^{-3}(\bullet), 3.5 \times$ $10^{-3}(\bullet), 3.0 \times 10^{-3}(\bullet), 2.5 \times 10^{-3}(\bullet)$. Xfoil simulation results are plotted as black diamonds $(\diamond)$, the measurements of [Gregory and OReilly, 1973] for Re $=1.44 \times 10^{6}$ for a rough foil $(-)$, and smooth foil (- - $)$, and the measurements of [Sheldahl and Klimas, 2006] at $\operatorname{Re}=1.36 \times 10^{6}$ for a smooth foil $(---)$. . 
16 Negative pressure coefficients $\left(C_{p}\right)$ plotted on the surface of the NACA0012 foil, calculated at $\operatorname{Re}=1.44 \times 10^{6}$ for $\theta=0^{\circ}(\mathrm{a})(\mathrm{c})$ and $\theta=8^{\circ}(\mathrm{b})(\mathrm{d})$. Xfoil results $(-)$ are compared to pLBM results for $(\Delta x / C)=2.5 \times 10^{-3}$ with (a) and (b) showing the perturbation component of the pressure $p^{P}$, calculated in the pLBM $(-)$ which is driven by its inviscid counterpart $p^{P}(-)$ and with (c) and (d) showing the total pressure, $p^{T}=p^{I}+p^{P},(-) \ldots \ldots \ldots \ldots$

17 Visualization of the hVOF with (a) a scenario when the total free surface is above the inviscid one, $\eta^{T}>\eta^{I}$, illustrating the perturbation NS domain (red) and a region where the total NS is solved (blue). (b) a scenario when the total free surface is below the inviscid one, $\eta^{T}<\eta^{I}$ and the dynamic free surface boundary condition is modified in the pLBM. . . . . . . . . . . . .

18 (a) Scenario when $\eta^{P}>0$ illustrating the interface between the perturbation NS domain and the total NS domain. (b) 2D representation of DFs crossing the perturbation $\mathrm{NS}$ and total NS interface. . . . . . . . . . . .

19 Snapshots of the gridded domain around a JHSS hull with (top) fluid cells shaded blue, turbulent wall model boundary cells shaded red, and the JHSS hull surface in light grey. (bottom) y-component of the normal vectors plotted for the same JHSS domain. . . . . . . . . . . . . . . . . . . . . .

20 Coefficient of lift $\left(C_{L}=F\left(1 / 2 \rho U^{2} C S\right)^{-1}\right)$ calculated using the LBM with the turbulent wall model $(\bullet)$, the pLBM with the turbulent wall model and analytical inviscid solution $(\bullet)$, and the pLBM with the turbulent wall model and BEM inviscid solution $(\bullet)$, and compared to measurements $(-)[1] \ldots$

21 (top) A visualization of the pLBM domain used in the JHSS steady resistance simulation, black lines in the image represent the outer extents of each grid and the hull is seen in orange. (bottom) pLBM domain parameters for the JHSS steady resistance simulation. . . . . . . . . . . . . .

(a) Visualization of the free surface elevation predicted by the BEM solver Aegir using a linear free surface at Fn $=0.3$. The JHSS hull can be seen in grey and for visualization purposes the sufaces are mirrored about the $\mathrm{y}=0$ plane. (b) Relevant non-dimensional physical parameters of each hybrid method run for the JHSS steady resistance simulations. . . . . . . . . . . . . .

23 Snapshots of the JHSS steady resistance simulation using the hybrid method running at a $\mathrm{Fn}=0.3$. with the hybrid domain cut along $y=0$ and $z=$ 0. (top) Visualization of the total hybrid method solution, with the linear free surface elevation (BEM) shown at $z=0$ and total velocity magnitude $u_{i}=u_{i}^{I}+u_{i}^{P}(\mathrm{BEM}+\mathrm{pLBM})$ shown along the $y=0$ plane, and the JHSS hull seen in grey. (bottom) Visualization of the perturbation velocity magnitude $u_{i}^{P}$ (pLBM) the domain is cut along the $y=0$ plane and only water and turbulent wall model nodes are shown. . . . . . . . . . . . . . .

24 Total steady resistance for the JHSS hull as a function of Froude number. Measurements are shown as blue circles $(\bullet)$, the resistance from wave generation $(\mathrm{BEM})$ is shown as grey circles $(\bullet)$ and the hybrid method solution of wave drag $(\mathrm{BEM})$ and viscous drag $(\mathrm{pLBM})$ with viscous pressure removed is shown as the green diamonds $(\checkmark) \ldots \ldots \ldots \ldots$ 
25 Simulation setup for the towed hydrofoil experiments with a scale visualization the domain (top) where the turquoise region is water and the small white region represents the foil. . . . . . . . . . . . . . .

26 LBM simulation results for the towed hydrofoil and foil submergence depth of 0.193 meters (non-breaking in experiments). (top) Snapshot of the velocity magnitude within the domain. (bottom) Plot of the steady state free surface elevation with measured results $(-)$, nodes containing a volume fraction, $\epsilon$, $0.1<\epsilon<0.9(\bullet)$, and a spline fit of those points $(-) . \ldots \ldots \ldots$

$27 \quad$ LBM simulation results for the towed hydrofoil and foil submergence depth of 0.159 meters (breaking in experiments). (a) Snapshot of the velocity magnitude within the domain before breaking and the region in black is where the power dissipation will be calculated. Wave breaking visualized within the black region is seen at 3.0 seconds (b and c) and 3.5 seconds (d and e). Velocity magnitude is shown ( $\mathrm{b}$ and $\mathrm{d}$ ) with blue representing $\left|u_{i}\right|=0.0 \mathrm{~m} / \mathrm{s}$ and red representing $\left|u_{i}\right|=1.5 \mathrm{~m} / \mathrm{s}$. The power dissipation is shown ( $\mathrm{b}$ and $\mathrm{d}$ ) with blue representing $0.0 \mathrm{~W}$ and red representing representing $1.0 \times 10^{-5} \mathrm{~W}$ calculated within each cell containing a volume of $3.4 \times 10^{-9} \mathrm{~m}^{3} \ldots \ldots$. . .

28 Snapshots of the hVOF simulation of a nonlinear wave using the a fully nonlinear potential flow solution and periodic side boundaries. The solution is shown at simulation start up (a) and at $t=0.9 T$ (b) as a balance between the driving inviscid flow and dissipative effects in the pLBM is reached. Cells shown have a volume fraction of $\epsilon \geq 0.5$ and color contours represent total velocity magnitude $\left(\mathbf{u}=\mathbf{u}_{i}^{I}+\mathbf{u}_{i}^{P}\right)$ with blue representing $|\mathbf{u}|=0.0 \mathrm{~m} / \mathrm{s}$ and red representing $\mathbf{u}=1.8 \mathrm{~m} / \mathrm{s} . \ldots \ldots \ldots \ldots \ldots \ldots$

29 Snapshots of the hVOF simulation of a nonlinear wave interacting with a cylinder (grey) using the a FNPF inviscid wave solution, periodic side boundaries and the cylinder boundary condition is applied in the pLBM. The solution is shown at quarter period $(T)$ intervals starting at time $t=T / 4(\mathrm{a})$ and ending at $t=T(\mathrm{~d})$. Cells shown have a volume fraction of $\epsilon \geq 0.5$ and color contours represent total velocity magnitude $\left(\mathbf{u}=\mathbf{u}_{i}^{I}+\mathbf{u}_{i}^{P}\right)$ with blue representing $|\mathbf{u}|=0.0 \mathrm{~m} / \mathrm{s}$ and red representing $\mathbf{u}=2.0 \mathrm{~m} / \mathrm{s} \ldots \ldots \ldots \ldots$ 


\section{List of Tables}

Table

Page

1 Convergence test results of the mean drag coefficient $C_{D}$, RMS of the lift coefficient $C_{L}^{\prime}$, and Strouhal number, as a function of the spatial resolution $\Delta x / D$ (in nested Grid 2), in the LBM and Hybrid pLBM simulations of a viscous flow past a circular cylinder of diameter $D \ldots \ldots \ldots \ldots$. . . .

2 Convergence test results of the mean drag coefficient $C_{D}$, RMS of the lift coefficient $C_{L}^{\prime}$, and Strouhal number, as a function of the Mach number, in the LBM and Hybrid pLBM simulations of a viscous flow past a circular cylinder of diameter $D \ldots \ldots \ldots \ldots \ldots \ldots \ldots \ldots$

3 Comparison of flow quantities with earlier results, for a viscous flow past a circular cylinder of diameter $D \ldots \ldots \ldots \ldots \ldots \ldots$. . . . . . . . . . . . . . . . .

4 Grid parameters used for the pLBM simulation of a foil coupled to a BEM solver. . . . . . . . . . . . . . . . . . . . . 


\title{
CHAPTER 1
}

\section{A lattice-Boltzmann-based perturbation method}

\author{
Christian F. Janßen ${ }^{a}$, Christopher M. O'Reilly ${ }^{b, c}$, Stephan T. Grilli ${ }^{b}$ \\ ${ }^{a}$ Institute for Fluid Dynamics and Ship Theory, Hamburg University of Technology, Hamburg, Germany \\ ${ }^{b}$ Department of Ocean Engineering, University of Rhode Island, Narragansett, RI, USA \\ ${ }^{c}$ Navatek Ltd., South Kingstown, RI, USA
}

This manuscript will be submitted for review to the Journal of Computers and fluids. 


\section{Abstract}

In this work, we report on the development and initial validation of a new hybrid numerical model for the simulation of complex wave-structure interactions. A kinetic Lattice Boltzmann method (LBM) model using a reduced domain is nested within an inviscid flow field to provide increased simulation fidelity where desired, while leveraging the computational efficiency of inviscid solutions. We formulate a fully (or strongly) coupled approach, in which a Helmholtz decomposition is applied to the flow, separating the inviscid and viscous perturbation parts. The latter component is driven by the inviscid field through nonlinear inviscid-perturbation interaction terms that, in conventional Navier-Stokes solvers, would be expressed as volume forces. In the present work an equivalent LBM approach is presented where, as opposed to a body-force coupling, a strong coupling within the LBM collision operators is presented. The resulting hybrid LBM is applied to validation cases for a wave driven boundary layer and the flow past a cylinder.

\subsection{Introduction}

Numerical models simulating the irrotational motion of an incompressible, inviscid fluid, based on potential flow theory, are computationally efficient and sufficiently accurate to simulate many engineering fluid problems, such as those involving free surface waves and wave-structure interactions (e.g., [16]). However, potential flow models cannot be used in applications where viscous effects are important, for instance, in the boundary layer near solid boundaries, or the ocean bottom, in the wake of bluff bodies, or to simulate surface wave breaking. Standard Computational Fluid Mechanics (CFD) Navier-Stokes (NS) solvers, such as based on a finite volume (e.g., [26]) or Lattice Boltzmann (LBM) method (e.g., [25, 10, 33, 13, 27, 28]), can model these types of flows, but are computationally costly. Additionally, for free surface flows, NS solvers often use a dissipative numerical scheme to capture the free surface boundary conditions, which is often too numerically dissipative to model wave propagation over long distances [6].

To more efficiently solve a broad class of hydrodynamics problems of interest to many engineering disciplines, in this work, we detail the development of a high-fidelity but low cost hybrid numerical model, that combines potential flow and NS models, and applies each model in the region where it is most effective. This hybrid model is based on a perturbation method proposed in earlier work, but for different numerical methods and problems [1, 15]. For instance, it was successfully used to model turbulent flows, using a finite volume method, and validated for turbulent channel and wave induced boundary layer flows [24] or for linear ship seakeeping 
([47]). Unlike one- or two-way coupled models applied over separate regions of the computational domain (e.g., $[6,22])$, in this method, both the velocity and pressure fields are expressed as the sum of inviscid/irrotational $(I)$ and viscous perturbation $(P)$ components, each solved using different numerical models in separate but overlapping computational domains. This method is sometimes referred to in fluid mechanics as the Helmholtz decomposition. More specifically, the $I$ fields are solved with a potential flow model typically over a larger size domain extending to the far-field, whereas the $P$ fields are solved based on a modified (perturbed) NS equation, here with a LBM model, in a smaller near-field domain in which viscous effects are deemed important based on the considered problem (this will be made more clear later). Thus, the more computationally demanding perturbation LBM model, referred to as pLBM, is only used in the smaller near-field domain where viscous/turbulent effects matter, with its solution forced by results of the potential flow model applied to the larger domain. Hence this hybrid approach is much more computationally efficient than applying a LBM model to the entire domain, while ensuring that the complete NS solution is solved where the physics calls for it.

In engineering applications involving complex boundary conditions and/or boundary/structure geometry, the model solving potential flow equations over the entire computational domain must itself be an optimized generic numerical solver, such as based on the higher-order Boundary Element Method, and feature fully nonlinear free surface boundary conditions if applicable $[29,24]$. Such cases, however, are not considered here but left out for follow-up publications $[42,43]$. The present paper instead concentrates on detailing the development of a novel pLBM model and validating it on a series of applications for which there are analytical solutions of the potential flow fields $I$ that can be used in the hybrid model to force the pLBM solution.

In our work, we use a LBM to solve NS equations, instead of a finite volume solver as in earlier work, in part because the data locality and kernel simplicity of the LBM allow for a very efficient parallel implementation of the model on a "General Purpose Graphical Processor Units" (GPGPU) [30, 51, 52]. While a single GPGPU still has a limited memory, a multi-GPGPU implementation of the LBM may achieve a higher computational efficiency, for an identical accuracy, than traditional CFD solvers implemented on a massively parallel CPU cluster. In the hybrid method context, for many engineering applications, the reduced-size pLBM computational domain can often be simulated using a single GPGPU [43], allowing simulation to be run on a desktop computer equipped with a relatively inexpensive GPGPU co-processor. When the potential flow is also solved with a numerical model (e.g., BEM based) its solution may then 
be calculated using the computer's often parallelized CPUs, with limited conflicting resource requirements. If a traditional NS solver were to be used in place of the LBM, a significant number of CPU nodes would be required to run it at an accuracy equivalent to that of the LBM, leading to competing computational resources when combined with the potential flow solver.

The coupling between continuum mechanics-based equations (or models), such as potential flow, and the kinetic-based LBM is less straightforward than the earlier implementation of the hybrid method based on a volume of fluid NS solver [24]. In particular, one must derive a pLBM equivalent to the nonlinear $I-P$ coupling terms that appear in the perturbation NS equations (see details below). To assess the ability of the LBM to simulate strongly nonlinear free surface flows, Janssen et al. [28, 29, 30] simulated the two-dimensional (2D) "weak coupling" wave breaking results reported in earlier work [6, 22], using a LBM in combination with a Volume Of Fluid (VOF) interface tracking method. In such cases, the LBM model was simply initialized with potential flow results for waves that had been propagated up to the breaking point in a potential flow BEM model $[18,19,20]$. Next, the same authors computed similar results with the hybrid method, in which the $I-P$ coupling terms were represented as LBM body force terms, using the pre-computed $I$ fields to force the $P$ field solution through these terms. This approach, while proven effective, required computing spatial derivatives of both the $I$ and $P$ fields using finite difference approximations that yielded a compact but non-local LBM kernel. Additional analyses showed that this approach both caused higher truncation errors in the pLBM than in the original LBM collision operator and reduced the overall efficiency of the parallelized GPGPU solution. Therefore, Janssen [28] suggested instead to introduce the nonlinear $I-P$ coupling terms directly into the LBM equilibrium probability distribution functions (EPDFs), hence, to develop perturbation EPDFs or pEPDFs. The latter were incrementally developed, implemented, and validated as part of the development of a pLBM model component to a hybrid naval hydrodynamic solver, in which the potential flow solution, with fully nonlinear free surface boundary conditions (FNPF), was computed using a higher-order BEM model [41, 42, 43].

In this paper, we fully report on the rigorous development, and mathematical and numerical validation of the pEPDFs in an efficiently parallelized pLBM, implemented on a GPGPU as a component of a hybrid hydrodynamic solver. Janssen's original approach [28] may be considered as a "top down" method, because the EPDFs were empirically derived based on the desired macroscopic quantities, i.e., the perturbation NS equations they were meant to represent. Here, instead, a "bottom-up" derivation of the collision operators is carried out to derive the pEPDFs, 
through applying the Helmholtz decomposition directly to the PDFs. While we recover the same pEDPFs as in [28], we also provide a rigorous proof that these indeed solve the perturbation NS equations, through a Chapman-Enskog expansion. The appropriate initial conditions, boundary conditions, and grid refinement considerations for the pLBM are then discussed, and the threedimensional (3D) method is validated, but here only for standard 2D applications, for which accuracy and convergence properties are demonstrated; three-dimensional applications will be reported elsewhere.

\subsection{Lattice Boltzmann Method (LBM)}

In part due to its efficiency, models based on the LBM have become increasingly widely used for solving a variety of complex fluid dynamics and multi-fluid multi-physics problems (e.g., $[2,3,4])$. By contrast with classical CFD solvers that model the macroscopic NS equations on a continuum basis, the LBM simulates CFD problems on a mesoscopic scale, in which the fluid is represented by the PDFs of discrete particles moving on a fixed lattice. Macroscopic hydrodynamic quantities are obtained from low-order moments of the PDFs. He and Luo, [25], Lallemand and Luo [34], and d'Humieres et al. [10] discuss the LBM theory.

Besides its numerical efficiency, significant advantages of the LBM are that it exactly satisfies mass conservation and, being a pseudo-compressible method, for a single fluid there is no need to solve a pressure Poisson equation, which is typically the most time consuming part of CFD solvers. A disadvantage is the LBM low order of convergence of numerical errors (secondorder), consistent with the Chapman-Enskog expansion, which requires using smaller spatial and temporal discretization sizes than for standard CFD solvers, to achieve a similar numerical accuracy. This, however, is typically compensated for by the LBM's excellent scalability on massively parallel computer hardware [11]; GPGPU implementations of the LBM have achieved remark-

able performances [51, 52,30]. Geller et al. [13] present a study of transient laminar flows, as compared to solutions of standard CFD solvers, and Krafczyk et al. [33] discuss Large Eddy Simulations (LES), both demonstrating the efficiency and accuracy of the LBM in these contexts.

\subsubsection{LBM fundamentals}

The primary variable of microscopic kinetic approaches is the PDF $f(t, \boldsymbol{x}, \boldsymbol{\xi})$, which specifies the normalized probability to encounter a particle at position $\boldsymbol{x}$ at time $t$, with velocity $\boldsymbol{\xi}$. The 
PDF evolution is described by the Boltzmann equation,

$$
\frac{D f}{D t}=\frac{\partial f(t, \boldsymbol{x}, \boldsymbol{\xi})}{\partial t}+\boldsymbol{\xi} \cdot \frac{\partial f(t, \boldsymbol{x}, \boldsymbol{\xi})}{\partial \boldsymbol{x}}=\Omega+\boldsymbol{B},
$$

whose left-hand side is an advection-type expression, the collision operator $\Omega$ describes particle interactions at the microscopic scale, and $\boldsymbol{B}$ represents body force effects.

A computationally efficient 3D model based on the Boltzmann Eq. (1) is first obtained by introducing a discretization in the velocity space $\xi$, and the resulting discrete Boltzmann equations,

$$
\frac{D f_{\alpha}}{D t}=\frac{\partial f_{\alpha}(t, \boldsymbol{x})}{\partial t}+\boldsymbol{\xi}_{\alpha} \cdot \frac{\partial f_{\alpha}(t, \boldsymbol{x})}{\partial \boldsymbol{x}}=\Omega_{\alpha}+B_{\alpha} .
$$

In this work we solve Eq. (2) on the commonly used D2Q9 and D3Q19 lattices. The former uses 9 lattice vectors or lattice links, to model 2D flows by connecting a node to its neighbors with $\boldsymbol{e}_{\alpha}=\{0,0\},\{ \pm c, 0\},\{0, \pm c\},\{ \pm c, \pm c\}, \alpha=0, \ldots, 9$ and the latter, a 3D model, contains 19 vectors $\boldsymbol{e}_{\alpha}=\{0,0,0\},\{ \pm c, 0,0\},\{0, \pm c, 0\}\{0,0, \pm c\},\{ \pm c, \pm c, 0\},\{ \pm c, 0, \pm c\},\{0, \pm c, \pm c\}, \alpha=$ $0, \ldots, 18$ [45], with a constant velocity $c$ representing the speed of particle propagation on the lattice. Eq. (2) is now discretized in space and time using a standard first-order finite difference scheme, which yields the lattice Boltzmann equations,

$$
f_{\alpha}\left(t+\Delta t, \boldsymbol{x}+\boldsymbol{e}_{\alpha} \Delta t\right)-f_{\alpha}(t, \boldsymbol{x})=\Omega_{\alpha}+B_{\alpha}
$$

with $\Delta t$, the temporal resolution. In the LBM, this equation is divided into a nonlinear collision step, which drives the PDFs towards a local equilibrium, and a non-local linear propagation step, where the post-collision PDFs $\left(\bar{f}_{\alpha}\right)$ are advected to neighboring nodes as,

$$
\bar{f}_{\alpha}(t, \boldsymbol{x})=f_{\alpha}(t, \boldsymbol{x})+\Omega_{\alpha}+B_{\alpha} \quad \text { and } \quad f_{\alpha}\left(t+\Delta t, \boldsymbol{x}+\boldsymbol{e}_{\alpha} \Delta t\right)=\bar{f}_{\alpha}(t, \boldsymbol{x})
$$

respectively. For numerical efficiency a mesh Courant number of $C_{o}=1$ is chosen to remove the need for finite differencing after the propagation step, and a relationship between the spatial $(\Delta x)$ and temporal discretization is found as $\Delta x=e_{\alpha} \Delta t$. It has been well-established in the literature that, with the proper choice of the collision operator (see next section), the solution of the lattice Boltzmann Eqs. (3) converge to that of the incompressible NS equations to within $\mathcal{O}\left(\Delta x^{2}\right)$ and $\mathcal{O}\left(\mathrm{Ma}^{2}\right)[12,31]$.

Macroscopic values of the hydrodynamic pressure $p=c_{s}^{2} \rho$ (assuming an ideal gas and speed of sound, $c_{s}$ ) and fluid velocity $\boldsymbol{u}$ are then found from hydrodynamic moments of the PDFs as,

$$
p(\boldsymbol{x}, t)=c_{s}^{2} \rho(\boldsymbol{x}, t)=c_{s}^{2} \sum_{\alpha=0}^{Q} f_{\alpha}(\boldsymbol{x}, t) \quad \text { and } \quad \boldsymbol{u}(\boldsymbol{x}, t)=\frac{1}{\rho} \sum_{\alpha=0}^{Q} \boldsymbol{e}_{\alpha} f_{\alpha}(\boldsymbol{x}, t)
$$


with $Q=9,18$ for our 2D and 3D lattices, respectively.

Before continuing we first consider the lattice scaling and discrete lattice effects associated with our numerical scheme. To enforce isotropy, lattice dependent weighting factors $w_{\alpha}$ (seen in Eq. (10)) are introduced as a result of using different lattice lengths $\boldsymbol{e}_{\alpha}$ [35]. For the D2Q9 model these are

$$
w_{0}=\frac{4}{3}, \quad w_{1 . .4}=\frac{1}{9} \quad \text { and } \quad w_{5 . .8}=\frac{1}{36}
$$

and for the D3Q19 model [25],

$$
w_{0}=\frac{1}{3}, \quad w_{1 . .6}=\frac{1}{18} \quad \text { and } \quad w_{7.18}=\frac{1}{36} .
$$

Both of these choices give the relationship between the speed of sound, $c_{s}$, and particle velocity, $c$, as $c_{s}^{2}=c / 3$.

We now consider the scaling of our physical variables to non-dimensional lattice variables (denoted by prime variables in the following) using spatial, temporal, and mass scales $\lambda, \tau$, and $\varpi$, respectively. In LBM, with $C_{o}=1$, one typically assumes, $\Delta x^{\prime}=\Delta x / \lambda=1, \Delta t^{\prime}=\Delta t / \tau=1$, $c^{\prime}=c \tau / \lambda=1$, and $m^{\prime}=m / \varpi=1$. Accordingly, our physical variables are scaled based on the

flow Mach number $\mathrm{Ma}=u / c_{s}=u^{\prime} / c_{s}^{\prime}$ and Reynolds number, $\mathrm{Re}=u \ell / \nu=u^{\prime} \ell^{\prime} / \nu^{\prime}$ (with $\ell \mathrm{a}$ representative length scale of the flow) and our physical length scale, $\lambda$, becomes $\lambda=\Delta x / \ell$. Inserting $c_{s}^{\prime}=c^{\prime} / \sqrt{3}=1 / \sqrt{3}$ into the Mach number equation gives $u^{\prime}=\operatorname{Ma} \sqrt{3}$ and $\tau$ is found as $\tau=\lambda u / u^{\prime}$. Hence, the non-dimensional fluid viscosity reads, $\nu^{\prime}=\nu \tau / \lambda^{2}$. For simplicity, in the following, we will drop the prime notation for non-dimensional variables unless stated otherwise.

\subsubsection{Collision operators}

For modeling interactions between fluid particles, different collision operators $\Omega_{\alpha}$ have been proposed. In the single relaxation time (SRT) model [5], the PDFs are driven towards an equilibrium state (denoted by an $e q$ superscript) based on a single relaxation time $\tau=3 \nu / c^{2}+\Delta t / 2$, for which particle collisions are modeled as,

$$
\Omega_{\alpha}=-\frac{\Delta t}{\tau}\left\{f_{\alpha}(\boldsymbol{x}, t)-f_{\alpha}^{e q}(\rho, \boldsymbol{u})\right\}
$$

with,

$$
f_{\alpha}^{e q}(\rho, \boldsymbol{u})=w_{\alpha} \rho\left(1+\left(3 \frac{\left(\boldsymbol{u} \cdot \mathbf{e}_{\alpha}\right)}{c^{2}}+\frac{9}{2} \frac{\left(\boldsymbol{u} \cdot \mathbf{e}_{\alpha}\right)^{2}}{c^{4}}-\frac{3}{2} \frac{\boldsymbol{u}^{2}}{c^{2}}\right)\right)
$$


To ensure that the LBM solution will converge towards that of the incompressible NS equations, our equilibrium is modified to

$$
f_{\alpha}^{e q}(\rho, \boldsymbol{u})=w_{\alpha}\left(\rho+\rho_{o}\left(3 \frac{\left(\boldsymbol{u} \cdot \mathbf{e}_{\alpha}\right)}{c^{2}}+\frac{9}{2} \frac{\left(\boldsymbol{u} \cdot \mathbf{e}_{\alpha}\right)^{2}}{c^{4}}-\frac{3}{2} \frac{\boldsymbol{u}^{2}}{c^{2}}\right)\right)
$$

with variables $\rho_{o}$ and $\rho$ now representing the average fluid density and a small density perturbation, respectively. This modification allows convergence to the incompressible NS equations provided that the simulation Mach number is sufficiently small, Ma $=u^{\prime} / c_{s}^{\prime} \ll 1[25]$ (primes denote LBM scaled variables here). This removes the requirement that $\mathrm{Ma}=u / c_{s}=u^{\prime} / c_{s}^{\prime}$ which is important for an efficient hydrodynamic simulation, where physical Mach numbers are often very small, as physical time step $\Delta t=\Delta t^{\prime} / \tau$ increases with an increasing simulation Ma.

In the more advanced and accurate MRT model [10], the PDFs and EPDFs are transformed into moment space, where the PDFs are relaxed using several different relaxation rates (and times). MRT was shown to increase the stability of LBM models, particularly when applied to high Reynolds flows, which are of greater practical interest, and at the same time to enable the development of more accurate boundary conditions [14]. The moments used in the MRT $\mathbf{m}=\mathbf{M} \cdot \mathbf{f}$ are labeled as,

$$
\mathbf{m}=\left(\rho, e, \epsilon, j_{x}, q_{x}, j_{y}, q_{y}, j_{z}, q_{z}, 3 p_{x x}, 3 \pi_{x x}, p_{w w}, \pi_{w w}, p_{x y}, p_{y z}, p_{x z}, m_{x}, m_{y}, m_{z}\right)^{T}
$$

and denote the following: mass density $m_{0}=\rho$; the part of kinetic energy independent of density $m_{1}=e$; the part of kinetic energy square independent of density and kinetic energy $m_{2}=\epsilon$; momentum $m_{3,5,7}=j_{x, y, z} ; m_{4,6,8}=q_{x, y, z}$ are related to heat flux; $m_{9,11,13,14,15}$ are related to the symmetric traceless viscous stress tensor; $m_{16,17,18}$ are third-order moments; and $m_{10,12}$ are fourth-order moments. The collision operator for the MRT model is defined as,

$$
\boldsymbol{\Omega}=\mathrm{M}^{-1} \cdot \mathrm{S} \cdot\left(\mathrm{M} \cdot \mathbf{f}-\mathbf{m}^{e q}\right)
$$

where $\mathrm{M}$ denotes the transformation matrix from distribution functions to moments $(\mathbf{m}=\mathbf{M} \cdot \mathbf{f}$ and $\left.\mathbf{f}=\mathbf{M}^{-1} \cdot \mathbf{m}\right), m_{\alpha}^{e q}$ are equilibrium moments that are calculated from Eq. (10), and $\mathbf{S}=s_{\alpha, \alpha}$ is a diagonal collision matrix of relaxation parameters. The parameters,

$$
s_{9,9}=s_{11,11}=s_{13,13}=s_{14,14}=s_{15,15}=-\frac{\Delta t}{\tau}=s_{\omega}
$$

are related to the kinematic viscosity $\nu$ via the relaxation time $\tau$ as,

$$
\tau=3 \frac{\nu}{c^{2}}+\frac{1}{2} \Delta t
$$


The remaining relaxation parameters,

$$
\begin{aligned}
& s_{1,1}=s_{a}, s_{2,2}=s_{b}, s_{4,4}=s_{6,6}=s_{8,8}=s_{c}, \\
& s_{10,10}=s_{12,12}=s_{d} \quad \text { and } \quad s_{16,16}=s_{17,17}=s_{18,18}=s_{e} .
\end{aligned}
$$

can be tuned to improve the model stability [34]. While the optimal values of these parameters depend on the specific system under consideration (geometry, initial and boundary conditions), reasonable values are given in [10]. Here, we use $s_{a}=s_{b}=s_{c}=s_{d}=s_{e}=-1.0$.

\subsection{Perturbation Lattice Boltzmann Method (pLBM)}

Before developing the pLBM and perturbation EPDFs (pEPDFs), we first derive the macroscopic perturbation NS equations that we seek to solve with the pLBM. As a canonical target application for the pLBM, we consider the viscous perturbation caused by a solid body boundary, occurring in a localized region with an otherwise inviscid and incompressible flow region. In this paper, we only consider the direct NS simulation (DNS) of low Re problems, which eliminates the additional complexity of introducing a turbulence model to simulate high Re flows. However, the pLBM can and has been extended to include a LES subgrid-scale model [42, 43]; this will be detailed in a follow-up paper. Generally as Re increases the region where viscous effects are significant decreases, e.g., along a solid boundary, allowing to use a smaller simulation domain for the pLBM model and yielding an increased efficiency of the hybrid solver.

\subsubsection{Coupling approach through the hybrid NS equations}

Following, for instance the developments in [24], in the region where viscous effects are important, the pressure and velocity fields are decomposed into the inviscid part $\left(p^{I}, u_{i}^{I}\right)$ and the viscous perturbation $\left(p^{P}, u_{i}^{P}\right)$ (tensor notation with its summation convention is used thereafter) as,

$$
u_{i}=u_{i}^{I}+u_{i}^{P} \quad \text { and } \quad p=p^{I}+p^{P}
$$

In the hybrid method, $\left(p^{I}, u_{i}^{I}\right)$ are obtained from the solution of potential flow equations, while the LBM is applied to solve the resulting perturbation NS equations for the perturbation fields $\left(p^{P}, u_{i}^{P}\right)$. Inserting the decomposition Eqs. (14) into incompressible NS equations,

$$
\frac{\partial u_{i}}{\partial t}+u_{j} \frac{\partial u_{i}}{\partial x_{j}}=g_{i}-\frac{1}{\rho} \frac{\partial p}{\partial x_{i}}+\nu \frac{\partial^{2} u_{i}}{\partial x_{j} \partial x_{j}}
$$


with gravitational acceleration $g_{i}$, and removing Euler's equations for the $I$ fields, which they exactly satisfy, yields the perturbation momentum equations,

$$
\frac{\partial u_{i}^{P}}{\partial t}+u_{j}^{P} \frac{\partial u_{i}^{P}}{\partial x_{j}} \underbrace{+u_{i}^{I} \frac{\partial u_{i}^{P}}{\partial x_{j}}+u_{j}^{P} \frac{\partial u_{i}^{I}}{\partial x_{j}}}_{\text {Additional forcing terms }}=-\frac{1}{\rho} \frac{\partial p^{P}}{\partial x_{i}}+\nu \frac{\partial^{2} u_{i}^{P}}{\partial x_{j} \partial x_{j}}
$$

in which additional convection-like interaction terms between the $u_{i}^{I}$ and $u_{i}^{P}$ fields appear.

Inserting the decomposition Eqs. (14) into the mass conservation equation yields.

$$
\frac{\partial u_{i}}{\partial x_{i}}=\frac{\partial u_{i}^{I}}{\partial x_{i}}+\frac{\partial u_{i}^{P}}{\partial x_{i}}=0
$$

With the $I$ fields satisfying a divergence free equation, the perturbation NS equations read,

$$
\begin{aligned}
\frac{\partial u_{i}^{P}}{\partial x_{i}} & =0 \\
\frac{\partial u_{i}^{P}}{\partial t}+\frac{\partial}{\partial x_{j}}\left[u_{i}^{P} u_{j}^{P}+u_{i}^{I} u_{j}^{P}+u_{i}^{P} u_{j}^{I}\right] & =-\frac{1}{\rho} \frac{\partial p^{P}}{\partial x_{i}}+\nu \frac{\partial^{2} u_{i}^{P}}{\partial x_{j} \partial x_{j}} .
\end{aligned}
$$

These equations are solved by a perturbation LBM method, as detailed in the next section. Thus, at each time step, the velocity $u_{i}^{I}$ and its gradient obtained from the inviscid solution (i.e., potential flow model or analytical solution) are used to force the perturbation NS equations, and the perturbation velocity $u_{i}^{P}$ and its gradient are locally obtained from the moments of the LBM PDFs.

\subsubsection{Derivation of pLBM collision operators}

In deriving the pLBM equations, two approaches have been considered to incorporate the additional $I-P$ forcing terms of Eq. (16) into the standard LBM solution of NS equations. First, as detailed in $[28,29,30]$ these terms can be represented as momentum sources in the form of space- and time-dependent body forces, by way of $B_{\alpha}$ terms in Eq. (3), that are driving the simulation. Here, however, a second approach is developed to incorporate these terms directly into a modified collision operator $\Omega_{\alpha}$.

In the first approach, the $I$ field contributions are directly added at every time step to the PDFs $f_{\alpha}$, together with other contributions, such as from gravity and other volume forces. However, this approach was found not to converge well, or even to be unstable, when using the simplest $B_{\alpha}$ formulation corresponding to assuming spatially homogeneous body forces (e.g., such as gravity) [28]. For transient volume forces, as in the present hybrid model, an enhanced momentum source term should be used, that also considers effects of viscosity [23]. By contrast, the new formulation uses a modified momentum flux tensor, which as we shall see will have 
the significant advantage of representing effects of the inviscid-viscous coupling terms without the need for spatial derivatives of the $u_{i}^{I}$ or $u_{i}^{P}$ fields, thus eliminating the associated numerical errors.

In the following, the bottom-up derivation of a pLBM collision operator equivalent to the perturbation NS Eqs. (18) is detailed. In contrast to our earlier attempts [28], this derivation does not consider the macroscopic equations to deduce the necessary modifications in the LBM, but instead starts from a perturbation of the PDFs at the mesoscopic scale. The LBKG model will be discussed first, followed by a MRT formulation applicable to 2D and 3D flows.

The LBM PDFs are first divided into viscous and inviscid components as,

$$
f_{\alpha}(t, \boldsymbol{x})=f_{\alpha}^{I}(t, \boldsymbol{x})+f_{\alpha}^{P}(t, \boldsymbol{x})
$$

By definition, the inviscid part is assumed to be in equilibrium state with zero non-equilibrium,

$$
f_{\alpha}^{I}(t, \boldsymbol{x})=f_{\alpha}^{e q}\left(\rho^{I}, \boldsymbol{u}^{I}\right)
$$

Hence,

$$
f_{\alpha}(t, \boldsymbol{x})=f_{\alpha}^{e q}\left(\rho^{I}, \boldsymbol{u}^{I}\right)+f_{\alpha}^{P}(t, \boldsymbol{x})
$$

with $\rho^{I}=c_{s}^{-2} p^{I}(t, \boldsymbol{x})$ and $\boldsymbol{u}^{I}=\boldsymbol{u}^{I}(t, \boldsymbol{x})$.

\section{LBKG model}

According to the discrete SRT approach, the relaxation is modeled by a single relaxation time. Inserting the inviscid-viscid perturbation into Eqs. (8), we find,

$$
\begin{aligned}
\Omega_{\alpha} & =\frac{1}{\tau}\left[f_{\alpha}-f_{\alpha}^{e q}(\rho, \boldsymbol{u})\right] \\
& =\frac{1}{\tau}\left[f_{\alpha}^{I}+f_{\alpha}^{P}-f_{\alpha}^{e q}(\rho, \boldsymbol{u})\right] \\
& =\frac{1}{\tau}\left[f_{\alpha}^{e q}\left(\rho^{I}, \boldsymbol{u}^{I}\right)+f_{\alpha}^{P}-f_{\alpha}^{e q}(\rho, \boldsymbol{u})\right] \\
& =\frac{1}{\tau}\left[f_{\alpha}^{P}-\left(f_{\alpha}^{e q}\left(\rho^{I}+\rho^{P}, \boldsymbol{u}^{I}+\boldsymbol{u}^{P}\right)-f_{\alpha}^{e q}\left(\rho^{I}, \boldsymbol{u}^{I}\right)\right)\right]
\end{aligned}
$$

Due to nonlinearities in the definition of equilibrium PDFs in Eq. (10), the superposition of $I$ and $P$ fields does not yield a simple linear superposition of the $f_{\alpha}^{e q}$, but nonlinear interaction terms have to be considered. Thus,

$f_{\alpha}^{e q, P}=w_{\alpha}\left(\rho^{P}+\rho_{o}\left(3 \frac{\boldsymbol{u}^{P} \cdot \mathbf{e}_{\alpha}}{c^{2}}+\frac{9}{2} \frac{\left(\mathbf{e}_{\alpha} \cdot \boldsymbol{u}^{P}\right)^{2}+2\left(\mathbf{e}_{\alpha} \cdot \boldsymbol{u}^{P}\right)\left(\mathbf{e}_{\alpha} \cdot \boldsymbol{u}^{I}\right)}{c^{4}}-\frac{3}{2} \frac{\left(\boldsymbol{u}^{P}\right)^{2}+2 \boldsymbol{u}^{P} \cdot \boldsymbol{u}^{I}}{c^{2}}\right)\right)$. 
Based on this definition of the perturbation equilibrium PDFs, the first- and second-order moments of the equilibrium PDFs $f_{\alpha}$ yield,

$$
\begin{aligned}
\sum_{\alpha=1}^{Q} f_{\alpha}^{e q, P} & =\rho^{P} \\
\sum_{\alpha=1}^{Q} e_{\alpha i} f_{\alpha}^{e q, P} & =\rho_{o} u_{i}^{P} \\
\sum_{\alpha=1}^{Q} e_{\alpha i} e_{\alpha j} f_{\alpha}^{e q, P} & =p^{P} \delta_{i j}+\rho_{o} u_{i}^{I} u_{j}^{P}+\rho_{o} u_{i}^{P} u_{j}^{I}+\rho_{o} u_{i}^{P} u_{j}^{P}=\Pi_{\alpha \beta}^{(0)}
\end{aligned}
$$

with $\boldsymbol{\Pi}^{(0)}$ being the new leading order part of the momentum flux tensor $\boldsymbol{\Pi}$.

\section{MRT model}

The corresponding MRT collision operator $\boldsymbol{\Omega}$ of the pLBM, to use with Eq. (11), includes modified equilibrium moments for the momentum advection,

$$
\begin{aligned}
& m_{2}^{e q}=e^{e q}=3 \rho_{0}\left(\left(u_{x}^{P}\right)^{2}+\left(u_{y}^{P}\right)^{2}+2 u_{x}^{P} u_{x}^{I}+2 u_{y}^{P} u_{y}^{I}\right)-2 \rho \\
& m_{3}^{e q}=\epsilon^{e q}=-3 \rho_{0}\left(\left(u_{x}^{P}\right)^{2}+\left(u_{y}^{P}\right)^{2}+2 u_{x}^{P} u_{x}^{I}+2 u_{y}^{P} u_{y}^{I}\right)+\rho \\
& m_{8}^{e q}=p_{x x}^{e q}=\rho_{0}\left(\left(u_{x}^{P}\right)^{2}-\left(u_{y}^{P}\right)^{2}+2 u_{x}^{P} u_{x}^{I}-2 u_{y}^{P} u_{y}^{I}\right) \\
& m_{9}^{e q}=p_{x y}^{e q}=\rho_{0}\left(u_{x}^{P} u_{y}^{P}+u_{x}^{P} u_{y}^{I}+u_{y}^{P} u_{x}^{I}\right)
\end{aligned}
$$

for $2 \mathrm{D}$ cases, and,

$$
\begin{aligned}
& m_{1}^{e q, P}=e^{e q}=\rho_{0}\left(\left(u_{x}^{P}\right)^{2}+\left(u_{y}^{P}\right)^{2}+\left(u_{z}^{P}\right)^{2}+2 u_{x}^{P} u_{x}^{I}+2 u_{y}^{P} u_{y}^{I}+2 u_{z}^{P} u_{z}^{I}\right) \\
& m_{9}^{e q, P}=3 p_{x x}^{e q}=\rho_{0}\left(2\left(u_{x}^{P}\right)^{2}-\left(u_{y}^{P}\right)^{2}-\left(u_{z}^{P}\right)^{2}+4 u_{x}^{P} u_{x}^{I}-2 u_{y}^{P} u_{y}^{I}-2 u_{z}^{P} u_{z}^{I}\right) \\
& m_{11}^{e q, P}=p_{z z}^{e q}=\rho_{0}\left(\left(u_{y}^{P}\right)^{2}-\left(u_{z}^{P}\right)^{2}+2 u_{y}^{P} u_{y}^{I}-2 u_{z}^{P} u_{z}^{I}\right) \\
& m_{13}^{e q, P}=p_{x y}^{e q}=\rho_{0}\left(u_{x}^{P} u_{y}^{P}+u_{x}^{P} u_{y}^{I}+u_{y}^{P} u_{x}^{I}\right) \\
& m_{14}^{e q, P}=p_{y z}^{e q}=\rho_{0}\left(u_{y}^{P} u_{z}^{P}+u_{y}^{P} u_{z}^{I}+u_{z}^{P} u_{y}^{I}\right) \\
& m_{15}^{e q, P}=p_{x z}^{e q}=\rho_{0}\left(u_{x}^{P} u_{z}^{P}+u_{x}^{P} u_{z}^{I}+u_{z}^{P} u_{x}^{I}\right)
\end{aligned}
$$

for $3 \mathrm{D}$ cases. [Note that subscripts $x, y, z$ here correspond to subscripts $i=1,2,3$ elsewhere.] The remaining moments correspond to the standard MRT moments.

\subsubsection{Recovering the perturbation NS equations from the pLBM}

By applying a Chapman-Enskog (CE) expansion, the macroscopic behavior of a LBM model formulation can be found from a multi scaling analysis. An expansion parameter $\epsilon$ is introduced, which is proportional to the ratio of the lattice grid $\Delta x$ to a characteristic macroscopic length 
(e.g., $\ell$ ). In the following, the CE scaling analysis is applied to the pEPDFs (26), by way of a perturbation expansion, which shows that these indeed recover the perturbation NS Eqs. (18).

\section{CE expansion}

Let us first consider the following quantities and scales. For convenience of notations, time derivatives $\partial / \partial t$ are denoted as $\partial_{t}$ and spatial derivatives as $\partial / \partial x_{i}$ as $\nabla_{i}$. With $\epsilon=\Delta x / \ell \ll 1$, the PDFs are expanded as follows,

$$
f_{\alpha}=f_{\alpha}^{(0)}+\epsilon f_{\alpha}^{(1)}+\epsilon^{2} f_{\alpha}^{(2)}+\mathcal{O}\left(\epsilon^{3}\right) \quad \text { with } \quad \partial_{t}=\epsilon \partial_{t_{1}}+\epsilon^{2} \partial_{t_{2}}+\mathcal{O}\left(\epsilon^{3}\right) \quad \text { and } \quad \nabla_{i}=\epsilon \nabla_{i}
$$

With these definitions, the Taylor series expansion of the first term on the LHS of Eq. (3) reads,

$$
\begin{array}{r}
f_{\alpha}\left(t+\Delta t, x_{i}+e_{\alpha i} \Delta t\right)=f_{\alpha}\left(t, x_{i}\right)+\Delta t \epsilon\left(\partial_{t_{1}}+e_{\alpha i} \nabla_{i}\right) f_{\alpha}\left(t, x_{i}\right) \\
+\frac{\Delta t^{2}}{2} \epsilon^{2}\left(\partial_{t_{1}}+e_{\alpha i} \nabla_{i}\right)\left(\partial_{t_{1}}+e_{\alpha j} \nabla_{j}\right) f_{\alpha}\left(t, x_{i}\right)+\mathcal{O}\left(\epsilon^{3}\right)
\end{array}
$$

Defining $D_{\alpha}=\partial_{t_{1}}+e_{\alpha i} \nabla_{i}$, and collecting terms up to different orders yields,

$$
\mathcal{O}(1): \quad 0=-\frac{\Delta t}{\tau}\left(f_{\alpha}^{(0)}-f_{\alpha}^{e q}\right)
$$

to first order $[23,28]$ and thus $f_{\alpha}^{0}=f_{\alpha}^{e q}$.

The PDF components of $\mathcal{O}(\epsilon)$ or smaller are then defined as the non-equilibrium components of the PDFs $\left(f_{\alpha}^{n e q}\right)$, i.e.,

$$
\begin{gathered}
\mathcal{O}(\epsilon): \quad D_{\alpha} f_{\alpha}^{(0)}=-\frac{1}{\tau} f_{\alpha}^{(1)} \\
\mathcal{O}\left(\epsilon^{2}\right): \quad \partial_{t_{2}} f_{\alpha}^{(0)}+\frac{\Delta t}{2} \partial_{t 1} D_{\alpha} f_{\alpha}^{(0)}+\frac{\Delta t}{2} e_{\alpha i} \nabla_{i} D_{\alpha} f_{\alpha}^{(0)}+D_{\alpha} f_{\alpha}^{(1)}=-\frac{1}{\tau} f_{\alpha}^{(2)} .
\end{gathered}
$$

Substituting Eq. (33) into Eq. (34) yields,

$$
\mathcal{O}\left(\epsilon^{2}\right): \quad \partial_{t_{2}} f_{\alpha}^{(0)}+\left(1-\frac{\Delta t}{2 \tau}\right) D_{\alpha} f_{\alpha}^{(1)}=-\frac{1}{\tau} f_{\alpha}^{(2)}
$$

\section{PDF moments}

Computing the zeroth-order moment of Eq. (33) and considering the pEPDFs (26) recovers the conservation of mass equation for the perturbation NS equations,

$$
\begin{aligned}
\sum_{\alpha=1}^{n} \partial_{t_{0}} f_{\alpha}^{(0, P)}+\sum_{\alpha=1}^{n} e_{\alpha i} \partial_{i} f_{\alpha}^{(0, P)} & =-\frac{1}{\tau} \sum_{\alpha=1}^{n} f_{\alpha}^{(1, P)} \\
\partial_{t} \rho^{P}+\rho_{o} \nabla_{i} u_{i}^{P} & =0
\end{aligned}
$$


while the inviscid mass conservation equation is recovered when the inviscid form of the EPDFs of Eq. (10) are used,

$$
\rho_{o} \nabla_{i} u_{i}^{I}=0
$$

Taking the first-order moment of Eq. (33) and considering the pEPDFs (26) recovers the leading order terms of the perturbation NS equations,

$$
\begin{gathered}
\sum_{\alpha=1}^{n} e_{\alpha i} \partial_{t_{0}} f_{\alpha}^{(0, P)}+\sum_{\alpha=1}^{n} e_{\alpha i} e_{\alpha i} \nabla_{i} f_{\alpha}^{(0, P)}=-\frac{1}{\tau} \sum_{\alpha=1}^{n} e_{\alpha i} f_{\alpha}^{(1, P)} \\
\partial_{t} \rho_{o} u_{i}^{P}+\nabla_{i}\left(p^{P}+\rho_{o} u_{i}^{I} u_{j}^{P}+\rho_{o} u_{i}^{P} u_{j}^{I}+\rho_{o} u_{i}^{P} u_{j}^{P}\right)=0,
\end{gathered}
$$

and the inviscid momentum conservation equations (Euler equations) are recovered when the inviscid form of the EPDFs of Eq. (10) are used,

$$
\partial_{t} \rho_{o} u_{i}^{I}+\nabla_{i}\left(p^{I}+\rho_{o} u_{i}^{I} u_{j}^{I}\right)=0 .
$$

The latter confirms that Euler equations are exactly represented in the LBM when using the inviscid form of the EPDFs in Eq. (10), $f_{\alpha}^{e q, I}$. This is unlike NS or perturbation NS equations, in which non-equilibrium components of the EPDF's must be included to represent viscous effects. Therefore, in the hybrid modeling context, this implies that an inviscid potential flow field satisfying Euler equations can be exactly mapped to the LBM variables using $f_{\alpha}^{e q, I}$. Finally, this confirms that the decomposition method used to derive Eqs. (26) does not need to consider $f_{\alpha}^{n e q, I}$ or its moments, since these are zero by definition.

Based on these conclusions, one may infer that the numerical kinematic viscosity of the pLBM can be selected as identical to that of the standard LBM. This is confirmed by taking the first-order moment of Eq. (35), and then applying Eq. (26),

$$
\sum_{\alpha=1}^{n} e_{\alpha} \partial_{t 2} f_{\alpha}^{(0, P)}+\sum_{\alpha=1}^{n} e_{\alpha}\left(1-\frac{\Delta t}{2 \tau}\right)\left(\partial_{t_{0}}+e_{\alpha i} \nabla_{i}\right) f_{\alpha}^{(1, P)}=-\frac{1}{\tau} \sum_{\alpha=1}^{n} e_{\alpha} e_{\alpha} f_{\alpha}^{(2, P)}
$$

where the first moment of $f_{\alpha}^{(1, P)}$ is zero in the absence of a body force, and its second moment found by considering, $\epsilon \Pi^{(1, P)}=\Pi-\Pi^{(0, P)}$, with,

$$
\Pi^{(1, P)}=\sum_{\alpha=1}^{n} e_{\alpha i} e_{\alpha j} f_{\alpha}^{(1, P)}=-c_{s}^{2} \tau\left(\partial_{i} \rho_{o} u_{j}^{P}+\partial_{j} \rho_{o} u_{i}^{P}\right)
$$

and giving

$$
\partial_{t 2} \rho_{o} u_{i}^{P}-\nabla_{i}\left(\tau-\frac{\Delta t}{2}\right) c_{s}^{2}\left(\nabla_{j} \rho_{o} u_{k}^{P}+\nabla_{k} \rho_{o} u_{j}^{P}\right)=0
$$


The perturbation momentum conservation equations can now be recovered by considering Eqs. (38) and (40) to within $\mathcal{O}\left(\epsilon^{2}\right)$ and $\mathcal{O}\left(\mathrm{Ma}^{2}\right)$ as,

$$
\partial_{t} \rho_{o} u_{i}^{P}+\rho_{o} \nabla_{j}\left(u_{i}^{P} u_{j}^{P}+u_{i}^{P} u_{j}^{I}+u_{i}^{I} u_{j}^{P}\right)=-\nabla_{j} p^{P}+\nu \nabla_{j}^{2} u_{i}^{P}
$$

when the viscosity is defined as

$$
\nu=\left(\tau-\frac{\Delta t}{2}\right) c_{s}^{2}
$$

This confirms that the relaxation time of Eq. (13) is suitable for use in the pLBM.

\subsubsection{Implementation of the pLBM}

In addition to modifying the collision operator as detailed above, implementing the pLBM requires adapting both initial and boundary conditions for the PDFs, such that the sum of the $I$ and $P$ EPDFs satisfy that of the complete NS equations given by Eq. (10). In NS simulations with the LBM, the initial PDFs are typically specified by applying Eq. (10) to the initial values of the macroscopic flow velocity. In the pLBM, these EPDFs are replaced by the pEPDFs of Eq. (26), which can be used to specify initial PDFs, given initial values of the $I$ and $P$ macroscopic velocity fields. These and other modifications of the standard LBM scheme that are required to perform pLBM simulation are further detailed in the following subsections.

\section{Boundary conditions}

On a solid wall boundary, the inviscid flow velocity $\boldsymbol{u}_{w}^{I}$ must satisfy a no-flow condition in the normal direction to the wall, $\boldsymbol{u}_{w}^{I} \cdot \boldsymbol{n}=0$, while the total velocity $\boldsymbol{u}_{w}$ must satisfy both this condition and a no-slip condition along the wall, so that $\boldsymbol{u}_{w}=0$ at the wall. Hence, given Eq. (14), we have at the wall [24],

$$
\boldsymbol{u}_{w}^{P}=-\boldsymbol{u}_{w}^{I}
$$

In the LBM, this boundary conditions is expressed within the near-wall boundary layer (BL), using a standard bounce-back scheme for the post-collision PDFs $[7,14,30]$. For the pLBM, a modified bounce-back scheme is implemented for the PDFs as,

$$
f_{\alpha^{\prime}}^{P}\left(\boldsymbol{x}_{1}, t+\Delta t\right)=f_{\alpha}^{P}\left(\boldsymbol{x}_{1}, t\right)-2 \rho_{0} w_{\alpha} \frac{\boldsymbol{e}_{\alpha} \cdot\left(-\boldsymbol{u}_{w}^{I}\right)}{c^{2}}
$$

in velocity directions $\alpha$, crossing the wall boundary, and their opposite counterpart $\alpha^{\prime}$, at lattice nodes of coordinate $\boldsymbol{x}_{1}$ adjacent to the wall. This approach may be applied to more advanced 
bounce back schemes, for instance considering curved walls that are not lattice coincident [39]. Note, in the hybrid model, it is important to ensure that $\boldsymbol{u}_{w}^{I}$ is calculated on the solid boundary, i.e., at each intersection between the actual body surface and lattice links connecting body and fluid nodes.

\section{Nested lattice grids}

In the standard LBM, computational efficiency can be increased by using nested lattice grids of increasing resolution towards the solid boundary/body of interest. Information on PDFs is exchanged between grids along the nested lattice boundaries, following an acoustic scaling method that scales PDFs based on the corresponding grid and time step sizes [28]. In this method the speed of sound and Ma value are kept constant in all sub-grids, which yields grid-level-dependent relaxation times. For instance, a bisection of grid spatial resolution yields a sub-cycling of 2 in time step, since $c=$ const. $=1$ (in LBM scaled variables) and $\Delta t=\Delta x / c$, respectively.

Nested grids can also be used in the pLBM, provided some modifications are made to enforce the conservation of mass and momentum along the nested lattice boundaries. Assuming that, by construction, the forcing inviscid fields smoothly vary across nested grid boundaries, continuity of the perturbation NS equations is expressed at grid interfaces by dividing the PDFs entering a specific grid into their equilibrium and non-equilibrium components. The equilibrium components are calculated with Eq. (26) using values of the macroscopic fields $\rho^{P}, u_{i}^{P}$, and $u_{i}^{I}$ calculated before the PDFs are exchanged between two different grids. The non-equilibrium parts of the PDFs, $f_{\alpha}^{n e q}=f_{\alpha}^{n e q, P}=f_{\alpha}^{P}-f_{\alpha}^{e q, P}$, are then rescaled so that the PDFs entering a nested grid are defined as,

$$
f_{\alpha, f}^{P}=f_{\alpha}^{e q, P}+s_{c f}\left(f_{\alpha, c}^{P}-f_{\alpha}^{e q, P}\right) \quad \text { and } \quad f_{\alpha, c}^{P}=f_{\alpha}^{e q, P}+s_{f c}\left(f_{\alpha, f}^{P}-f_{\alpha}^{e q, P}\right)
$$

in which subscripts $f$ and $c$ denote the fine and coarse grids, respectively, and $s_{c f}$ and $s_{f c}$ are obtained by equating the total derivative of the PDFs [28],

$$
s_{c f}=\frac{6 \nu+\Delta t_{f}}{6 \nu+\Delta t_{c}} \quad \text { and } \quad s_{f c}=\frac{6 \nu+\Delta t_{c}}{6 \nu+\Delta t_{f}} .
$$

In addition to this scaling, an accurate interpolation of the PDFs in space and time must be performed to reconstruct hanging nodes in the fine lattice. This part of the nested grid method is not modified for the pLBM, for which further details can be found in [28]. 


\subsection{Applications}

The accuracy and convergence properties of the novel pLBM approach are assessed in the following, on the basis of a series of simple but meaningful applications. Although the model is implemented in $3 \mathrm{D}$, for the sake of validating the hybrid approach, only $2 \mathrm{D}$ applications are considered in this paper. More complex fully 3D cases will be presented in follow-up papers $[41,42,43]$.

To assess the basic convergence and accuracy properties of the proposed pLBM, we first consider two oscillatory laminar boundary layer (OLBL) problems in an incompressible, Newtonian (viscous) fluid, over a plane solid boundary, in which the inviscid forcing flow velocity is analytically defined as: (i) wall-parallel, uniform, and harmonic in time (Stokes OLBL problem); (ii) time- and space-dependent based on linear wave theory (Steady streaming problem in OLWBL). Then the viscous flow past a circular cylinder is simulated in the LBM and pLBM, where the pLBM is forced by a steady inviscid flow and the hybrid result of the inviscid and pLBM solutions produce a nearly exact agreement with the LBM.

\subsubsection{Oscillatory boundary layers over a plane solid wall Stokes boundary layer problem}

Stokes' second problem [50] provides an exact solution of NS equations for the horizontal velocity profile within an oscillatory laminar boundary layer (OLBL) over a plane solid wall in an incompressible Newtonian fluid, of density $\rho$ and dynamic viscosity $\mu$, forced by a periodic uniform wall-parallel inviscid velocity,

$$
u_{1}^{\prime}(z, t)=u_{0}\left(\sin (\omega t)-e^{-\frac{z}{\delta_{s}}} \sin \left(\omega t-\frac{z}{\delta_{s}}\right)\right)
$$

with $u_{0}$ the free-stream velocity magnitude, $\omega=2 \pi / T$ the angular frequency, $T$ the period, and $\delta_{s}=\sqrt{2 \nu / \omega}$ the Stokes-layer thickness, with $\nu=\mu / \rho$ the kinematic viscosity. A key feature of this OLBL velocity profile is that there is a $45^{\circ}$ phase lag between the free-stream velocity and the shear stress at the wall, $\tau_{w}=\mu \partial u_{1} / \partial z$, for $z=0$ (this is easily verified with Eq. (49)).

Harris and Grilli [24] solved this problem with a similar hybrid modeling approach, but using a finite volume solution of the perturbation NS equations, in which the $I-P$ forcing terms were represented as body forces. Here, we solve this problem with the pLBM model, using the modified collision operator based on the pEPDFs. Fig. 1 shows the numerical problem set-up and parameters. The hybrid domain height, in the cross-wall $z$-direction, is $H=10 \delta_{s}$ and its horizontal dimensions are $W=B=20 \delta_{s}$ in the $x$ - direction, which is quite small but adequate 


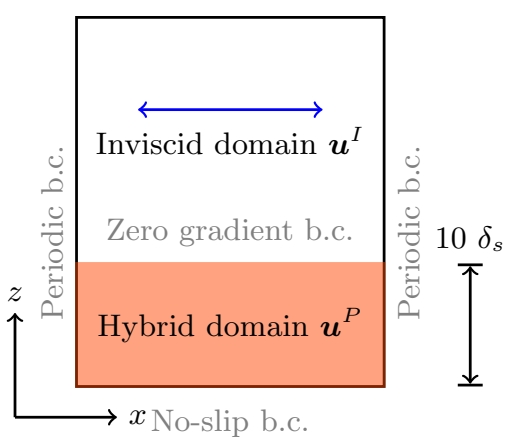

\begin{tabular}{cc}
\hline Param. & Value \\
\hline Domain height & $H=10 \delta_{s}$ \\
Upper boundary condition & Zero-gradient \\
Wall boundary condition & No-slip \\
Initial condition & $u_{i}^{P}\left(x_{i}, 0\right)=0$ \\
Forcing flow period & $T=1$ \\
OLBL Stokes length & $\delta_{s}=1$ \\
Kinematic viscosity & $\nu=\pi$ \\
Reynolds number & $\operatorname{Re}_{\delta}=1,2,3,10,100$ \\
\hline
\end{tabular}

Figure 1: (a) Domain setup for the Stokes OLBL simulation. The far-field periodic potential flow velocity is specified in the Inviscid domain, while the pLBM model is applied within the Hybrid domain. (b) Parameters used in numerical model applications

since the velocity is wall-parallel and spatially uniform [24].

The inviscid velocity specified in the hybrid model is,

$$
u_{1}^{I}(t)=u_{0} \sin (\omega t) \quad \text { with } \quad u_{2}^{I}=u_{3}^{I}=0 .
$$

with the complete solution given by,

$$
u(z, t)=u^{I}+u^{P}=u_{0} \sin (\omega t)+u^{P}
$$

A no-slip boundary condition for the complete velocity is specified on the wall $(z=0)$, and perturbation velocity is assumed to vanish at the top of the domain $\left(z=z_{\max }\right)$ (Fig. 1). Hence,

$$
\boldsymbol{u}^{P}(0, t)=-\boldsymbol{u}^{I}(t) \quad \text { and } \quad \partial_{z} f_{\alpha}^{P}\left(z_{\max }, t\right)=\mathbf{0} .
$$

Periodic boundary conditions for the PDFs are specified on lateral boundaries (in the $x$ and $y$ directions).

Simulations are run for 5 different free-stream velocity values, corresponding to Reynolds numbers $\operatorname{Re}_{\delta}=u_{0} \delta_{s} / \nu=1,2,3,10,100$, up to $t_{\max }=50 T$, for which steady state was achieved in all cases. In each of these cases, to assess the convergence properties of the pLBM, the solution is computed for 5 different hybrid domain discretizations, with $N_{z}=10,20,40,80$ or 160 nodes in the $z$-direction and $2 N_{z}$ nodes in the $x$ - direction. Diffusive scaling was used to determine the simulation Mach number for each $N_{z}$, meaning that a second order rate of convergence is expected when $\Delta \mathrm{Ma} \propto \Delta x^{2}$ [25] [35]. The corresponding Mach number values are Ma $=0.2$ in the coarsest mesh to 0.0125 in the finest mesh.

Fig. 2a shows velocity profiles based on the analytical solution of Eq. (49) at phase angles $\Phi=\omega t=0^{\circ}, 90^{\circ}, 180^{\circ}$, and $270^{\circ}$. In all cases, the accuracy of the numerical solution is assessed 
by computing the maximum $L_{1}$ norm of differences between the numerical and analytical solution over the entire hybrid domain, among these 5 phase angles, with

$$
L_{1}=\sum_{i}^{N_{z}} \frac{\left|u_{i}^{n}-u^{\prime}\left(z_{i}, t_{n}\right)\right|}{\left|u^{\prime}\left(z_{i}, t_{n}\right)\right|},
$$

vertical node $i$, time step $n$, and $u_{i}$ averaged over all nodes in the x-direction. Results are shown in Fig. 2b for each case, as a function of the respective discretization size $N_{z}$. The figure shows that for each $N_{z}$ value the maximum numerical error decreases with the Reynolds number, while for each Reynolds number numerical errors decrease with $N_{z}$; as expected for the LBM, the convergence rate is second-order with $1 / N_{z} \propto \Delta x$. This indicates that, to achieve a fixed error threshold (e.g., $5 \times 10^{-3}$ ), a finer discretization must be used, the larger the $\operatorname{Re}_{\delta}$ values.

Note that, in this simple application, due to the wall-parallel velocity field, the nonlinear $I-P$ convective terms in the left-hand-side of the perturbation NS Eq. (18) vanish by construction. Therefore, the same results should be obtained if the standard EPDFs of Eq. (10) are used instead of the pEPDFs of Eq. (26). This was verified in the OLBL results but will no longer be the case in other applications presented hereafter.

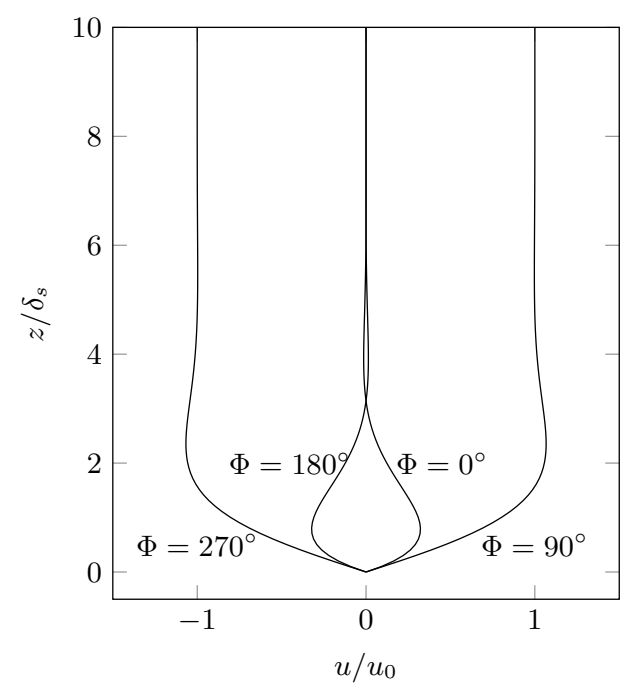

(a)

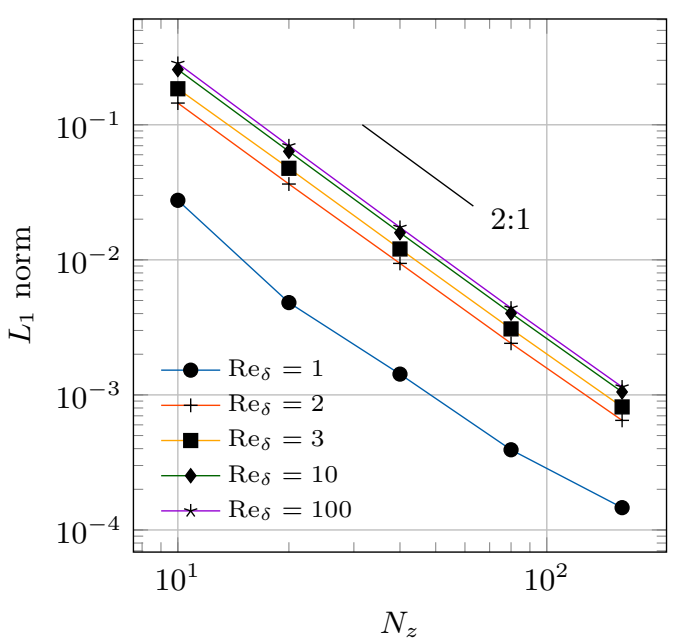

(b)

Figure 2: (a) Analytical horizontal velocity profile $u(z, t)$ within an OLBL [50], from Eq. (49); each line corresponds to a different phase angles $\Phi=\omega t$ (see Fig. 1 for physical parameters). (b) Maximum numerical error of the pLBM or LBM OLBL solution as a function of the Reynolds number $\operatorname{Re}_{\delta}$ and the discretization $N_{z}$; note the quadratic convergence rate with $1 / N_{z}$. 


\section{Steady streaming problem in OLWBL}

Here we consider an OLBL problem similar to that of the previous section, as sketched in Fig. 3a, but where the horizontal inviscid flow velocity forcing in the $x$ - direction is that of a linear periodic wave [9],

$$
u_{1}^{I}(x, z, t)=\frac{A g k}{\omega} \frac{\cosh k(z-h)}{\cosh k h} \cos (k x-\omega t) \quad \text { with } \quad u_{2}^{I}=0 ; \quad u_{3}^{I} \simeq 0,
$$

a wave amplitude $A$, wavenumber $k=2 \pi / L$, wavelength $L$, angular frequency $\omega=2 \pi / T$, wave period $T$, for a water depth $h$. Moreover, these parameters are related by the linear dispersion relationship,

$$
\frac{\omega^{2}}{g}=k \tanh k h
$$

The horizontal $x$-velocity $u_{1}^{I}$ varies in both the $x$ - and $z$-directions and, for long-crested waves propagating in the $(x, z)$ plane, the $y$-velocity $u_{2}^{I}=0$. In a linear ocean wave, the vertical velocity $u_{3}^{I}$ is non-zero away from the solid wall representing the seafloor. However, to derive an analytical solution within the BL, this velocity, which vanishes at the wall $(z=0)$, is assumed to be negligible within the $\mathrm{BL}$ and here also within the Hybrid domain. A non-zero velocity $u_{3}^{I}$ could of course be specified in the pLBM model, but there would no longer be an exact solution to compare the numerical results to. Unlike in the previous application, due to the spatial variation

of $u_{1}^{I}$ with $x$ and $z$, the $I-P$ nonlinear coupling terms in the perturbation NS Eq. (18) are non-zero. Therefore, this application is a true test of the accuracy of the pLBM solution, based on the pEPDFs of Eq. (26), computed based on the velocity field of Eq. (54).

As a more demanding test of accuracy of the hybrid model than just considering instantaneous velocity profiles as in the previous application, here we compute the steady streaming velocity within the OLWBL. This velocity is that of the period-averaged horizontal (wall-parallel) current that occurs within an OLWBL and was analytically derived by Longuet-Higgins [37] as,

$$
\begin{array}{r}
\bar{u}_{1}(\xi)=\frac{A^{2} \omega k}{\sinh ^{2} k h}\left[\frac{3}{4}-e^{-\xi} \cos \xi+\frac{1}{2} e^{-\xi} \sin \xi+\frac{1}{4} e^{-2 \xi}-\frac{1}{2} \xi e^{-\xi} \cos \xi-\frac{1}{2} \xi e^{-\xi} \sin \xi\right] \\
\text { and } \quad \bar{u}_{2}=\bar{u}_{3}=0
\end{array}
$$

with $\xi=(z-h) / \delta_{s}$ the non-dimensional distance to the wall. Note that additional contributions to the steady streaming velocity could also result from nonlinear effects, such as Stokes drift resulting from wave asymmetry.

As before, a no-slip bottom boundary condition, a zero vertical perturbation gradient at the upper boundary, and periodic lateral boundary conditions, are specified in the hybrid model (see 
(b)

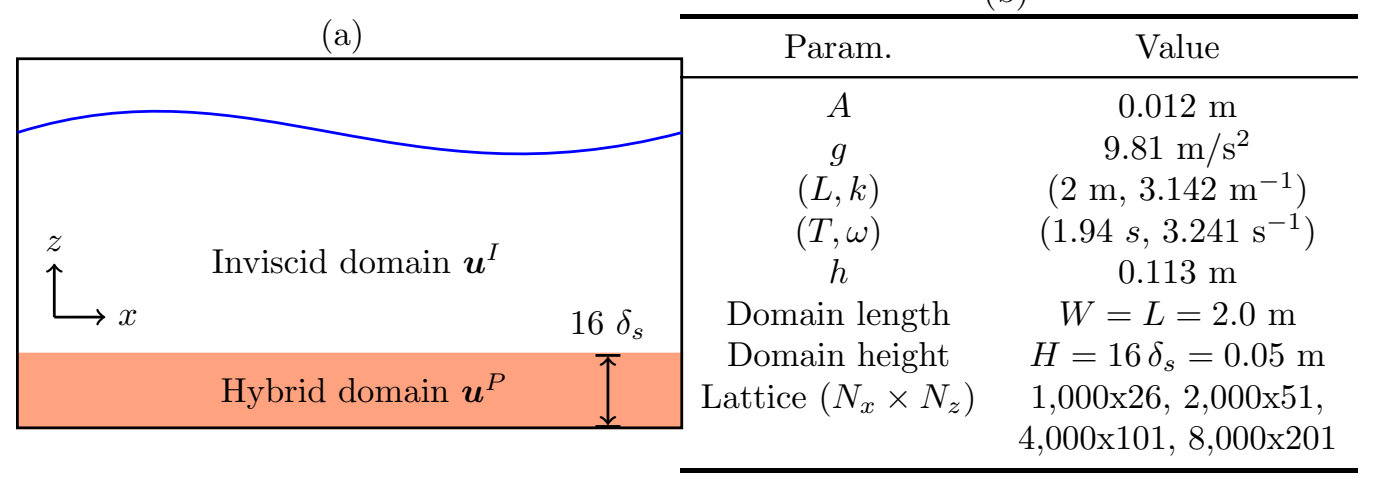

Figure 3: (a) Domain setup for the steady streaming OLWBL simulation. The far-field periodic potential flow velocity is specified in the Inviscid domain, while the pLBM model is applied within the Hybrid domain. (b) Parameters used in numerical model applications

Eqs. (52)). The domain set-up and simulation parameters are shown in Fig. 3 and the latter are selected such that a laminar flow is created in the BL. Specifically, with these parameters and assuming the fluid kinematic viscosity is set to $\nu=1.6 \times 10^{-5} \mathrm{~m}^{2} / \mathrm{s}$, we find $\delta_{s}=3.125 \times 10^{-3}$ $\mathrm{m}, 16 \delta_{s}=0.05 \mathrm{~m}$, as stated, and with the maximum flow velocity $u_{\max }=A g k / \omega=0.114$ $\mathrm{m} / \mathrm{s}$ we find $\operatorname{Re}_{\delta}=22.6$, which confirms that the $\mathrm{BL}$ flow is within the laminar regime. All simulations are run until a steady-state streaming velocity is achieved, based on a $L_{1}$ norm threshold $\Delta L_{1}<0.0025 u_{A}$, where $u_{A}$ is the vertical average of the analytical steady streaming solution, and $\Delta L_{1}$ is found by comparing the $L_{1}$ relative error norm of the calculated streaming velocity at times $t$ and $t-10 T$, thus $\Delta L_{1}=\left|L_{1}^{t}-L_{1}^{t-10 T}\right|$. In this application Eq. (53) is used to calculate $L_{1}$, with $\bar{u}$ replacing $u^{\prime}$.

Numerical results are computed with the pLBM for the physical parameters listed in Fig. 3b, using 4 different spatial discretization in which both the horizontal $\left(N_{x}\right)$ and vertical $\left(N_{z}\right)$ number of nodes in the lattice are increasing. Additionally, for each discretization, 4 different values of the Mach number are successively used, $\mathrm{Ma}=0.01,0.02,0.04,0.08$, which affects the time step. The steady streaming velocity profile is computed in each case and compared in normalized form to the analytical profile from Eq. (56), in 4. For each Ma value, except the largest, the figure shows that numerical results converge towards the analytical solution as grid resolution is increased. Convergence appears nearly identical and is fastest for the two lowest Ma values, whereas results diverge for the largest Ma value. Using the finest spatial $\left(N_{z}=201\right)$ and temporal $(\mathrm{Ma}=0.01)$ discretizations, numerical results are in very close agreement with the analytical results, with a relative error in terms of the $L_{1}$ norm of $0.57 \%$. Fig. 5 shows more 

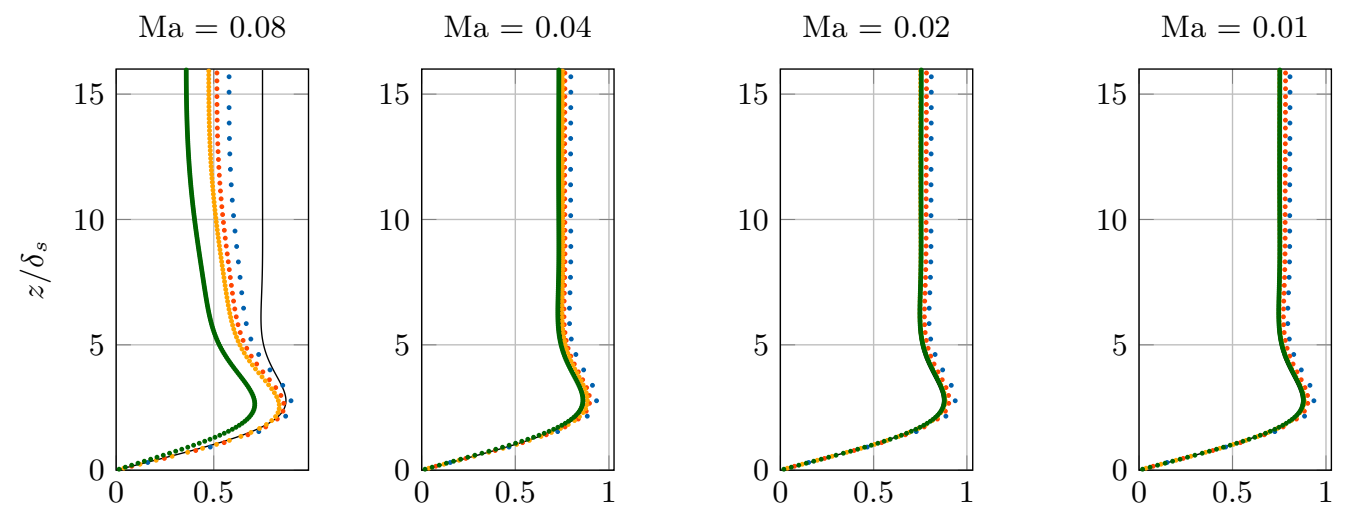

Figure 4: Normalized steady streaming velocity profile (i.e., period-averaged) $u^{*}=$ $\bar{u}_{1}\left(\frac{A^{2} \omega k}{\sinh ^{2} k h}\right)^{-1}$ in the OLWBL, for 4 different pLBM grids with vertical resolution $N_{z}=\delta_{s} / \Delta x=$

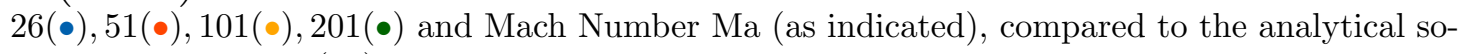
lution given by Eq. (56).

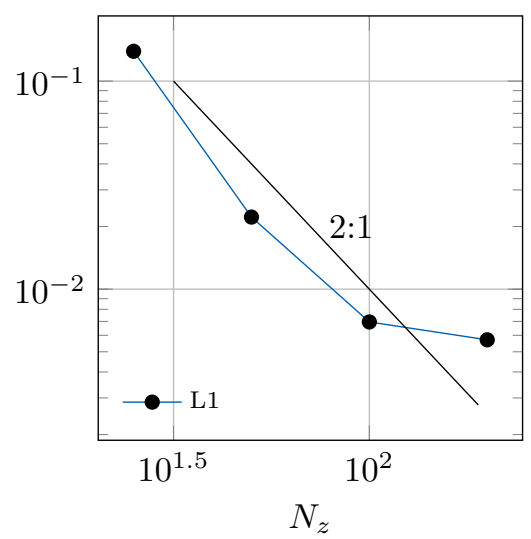

Figure 5: Convergence of numerical errors of the normalized period-averaged steady streaming velocity $u^{*}$, in the results of Fig. 4 , in terms of the $L_{1}$ (Eq. (53)) norms of the relative errors. Results shown are for successively increasing spatial and time resolutions as $\left(N_{z}, \mathrm{Ma}\right)=(26,0.08)$, $(51,0.04),(101,0.02)$, and $(201,0.01)$.

detailed results of the convergence of numerical errors as a function of the spatial and temporal discretization, in terms of the $L_{1}$ norms of the errors. As before, convergence is second-order with the discretization $\Delta x \propto 1 / N_{z}$.

Considering that the steady streaming velocity profile is an average over the wave period of much larger instantaneous velocities, this close agreement indicates that highly accurate results were achieved in the pLBM model.

\subsubsection{Viscous flow past a circular cylinder}

We consider the flow past a circular cylinder of diameter $D$ caused by a steady current, with spatially uniform free stream velocity $u_{1}^{I}=U$, in an incompressible Newtonian fluid of kinematic 
viscosity $\nu$, at a Reynolds number $\operatorname{Re}=U D / \nu=100$, for which a von Kármán vortex street occurs. As there is no analytical solution for this case, both the LBM and pLBM results will be compared with each other and with previously published results. The flow induces a mean drag force but, due to the von Kármán vortex street, an oscillatory lift force of zero mean. This application will demonstrate the ability of the pLBM model to perform DNS for highly separated flows, where the perturbation component of the flow $P$ is large compared to the inviscid forcing $I$ and also significantly unsteady. This application also features large spatial gradients of both the inviscid and hybrid solutions and curved boundaries over which the hybrid no-slip boundary condition is applied. Finally the ability of the pLBM to accurately predict forces on an solid body will be assessed.

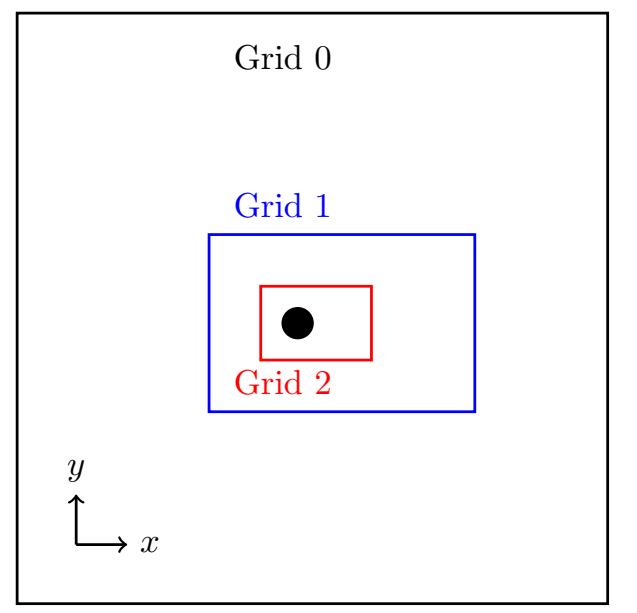

\begin{tabular}{cccc}
\hline$\#$ & $\begin{array}{c}\text { Min. Extent } \\
(x, y) / D\end{array}$ & $\begin{array}{c}\text { Dimensions } \\
(x, y) / D\end{array}$ & $\begin{array}{c}\text { Expansion } \\
\text { Ratio }\end{array}$ \\
\hline 0 & $(-50.0,-50.0)$ & $(100.0,100.0)$ & 8 \\
1 & $(-7.5,-7.5)$ & $(30.0,15.0)$ & 2 \\
2 & $(-2.0,-3.0)$ & $(8.0,6.0)$ & 1 \\
\hline
\end{tabular}

Figure 6: Nested domain set-up for the LBM and pLBM simulations of the viscous flow past a circular cylinder of diameter $D$, and associated geometric parameters for each grid; the expansion ratio indicates how the mesh size $\Delta x$ changes from one grid to the other. The cylinder center is located at $(0,0)$.

The LBM and pLBM domains are shown in Fig. 6. In this application, to achieve a higher efficiency, a series of refined nested grids are used, whose geometric characteristics are provided in the figure. The maximum extents of the domain is $50 D$ away from the center of the cylinder, which earlier work has shown is sufficient to make non-physical effects that the farfield boundaries might cause negligible $[36,49]$. In the LBM simulations, a specified free-stream velocity boundary condition is specified $(U)$ at the inlet of the larger domain $(x=-50 D)$ and on its sides $(y= \pm 50 D)$, while a zero gradient boundary condition for the PDFs in the $x$-direction is specified at the outlet boundary $(x=50 D)$; finally, a no-slip boundary condition is specified on the cylinder body based on Eq. (155), without the inviscid term. A uniform free-stream velocity 


\begin{tabular}{cccc|ccc}
\hline & $\mathrm{LBM}$ & & & Hybrid pLBM & & \\
$\Delta x / D$ & $C_{D}$ & $C_{L}^{\prime}$ & $\mathrm{St}$ & $C_{D}$ & $C_{L}^{\prime}$ & $\mathrm{St}$ \\
\hline $1 / 70$ & 1.341 & 0.235 & 0.165 & 1.389 & 0.240 & 0.162 \\
$1 / 140$ & 1.334 & 0.232 & 0.164 & 1.360 & 0.236 & 0.163 \\
$1 / 210$ & 1.333 & 0.232 & 0.164 & 1.347 & 0.232 & 0.164 \\
$1 / 280$ & 1.333 & 0.232 & 0.164 & 1.342 & 0.233 & 0.164 \\
$1 / 350$ & - & - & - & 1.339 & 0.232 & 0.164 \\
\hline
\end{tabular}

Table 1: Convergence test results of the mean drag coefficient $C_{D}$, RMS of the lift coefficient $C_{L}^{\prime}$, and Strouhal number, as a function of the spatial resolution $\Delta x / D$ (in nested Grid 2), in the LBM and Hybrid pLBM simulations of a viscous flow past a circular cylinder of diameter $D$.

and zero density fluctuation are used to initialize the PDFs.

In the hybrid model, equivalent boundary conditions are specified in the pLBM. The inviscid flow field is first calculated analytically based on the free-stream boundary condition $u_{1}^{I}=U$, using a conformal mapping method, and the perturbation PDFs are initialized assuming a zero perturbation flow field [43]. A zero gradient boundary condition of the perturbation PDFs is specified in the $x$-direction at the outlet boundary and Eq. (155) is used to specify the no-slip boundary condition on the cylinder body.

In the LBM simulations, the momentum exchange method is used to calculate forces on the cylinder [28]. In the hybrid model, the force acting on a solid body is decomposed into inviscid and perturbation components, $\mathbf{F}=\mathbf{F}^{I}+\mathbf{F}^{P}$. However, D'Alembert's paradox implies that inviscid forces are zero on the cylinder and only the perturbation component must be calculated, which is also done using the momentum exchange method. Based on this force, standard drag and lift coefficients are defined on the cylinder as, $C_{D}=F_{x} /\left(0.5 \rho D U^{2}\right)$ and $C_{L}=F_{y} /\left(0.5 \rho D U^{2}\right)$, respectively, assuming a unit length of cylinder.

Fig. 7 shows typical results of computations with the LBM model and the pLBM hybrid model. Visually, both solutions agree well with each other (subplots (a) and (b)). Tables 1 and 2 show numerical results as a function of the discretization, where $\Delta x / D$ represents the resolution of Grid 2 (Fig. 6). In all cases, steady state is reached and convergence of the solution is assessed in terms of the mean drag coefficient $C_{D}$, RMS of the lift coefficient $C_{L}$, referred to as $C_{L}^{\prime}$, and Strouhal number St $=f D / U$ where $f$ is the frequency of oscillation in $C_{L}$. Convergence with the spatial discretization (grid size) is investigated using a Mach number 0.1, while convergence in Mach number is investigated using $\Delta x / D=1 / 210$ and $\Delta x / D=1 / 350$ for the LBM and hybrid pLBM models, respectively.

Table 1 results indicate a quick and clear convergence of the LBM solution at $\Delta x / D=1 / 210$ 


\begin{tabular}{cccc|cccc}
\hline Mach & LBM & & & hybrid pLBM & & & \\
& $C_{D}$ & $C_{L}^{\prime}$ & St & Mach & $C_{D}$ & $C_{L}^{\prime}$ & St \\
\hline 0.2 & 1.334 & 0.237 & 0.163 & 0.2 & 1.342 & 0.237 & 0.163 \\
0.1 & 1.333 & 0.232 & 0.164 & 0.1 & 1.339 & 0.232 & 0.164 \\
0.05 & 1.333 & 0.232 & 0.165 & 0.05 & 1.339 & 0.232 & 0.164 \\
\hline
\end{tabular}

Table 2: Convergence test results of the mean drag coefficient $C_{D}$, RMS of the lift coefficient $C_{L}^{\prime}$, and Strouhal number, as a function of the Mach number, in the LBM and Hybrid pLBM simulations of a viscous flow past a circular cylinder of diameter $D$.

(with $\mathrm{Ma}=0.1$ ), but a significantly slower rate of convergence with the spatial resolution is observed for the hybrid pLBM results, particularly for $C_{D}$. This is likely the result of an imperfect cylinder boundary condition Eq. (155) in the pLBM. While the term $2 \rho_{0} w_{\alpha} \boldsymbol{e}_{\alpha} \cdot\left(-\boldsymbol{u}^{I}\right) / c^{2}$ does not increase the truncation error of the standard LBM wall boundary condition [7], an additional truncation error is introduced in the hybrid pLBM body boundary condition because here the inviscid velocity $u_{i}^{I}$ is calculated at each wall boundary node. Instead the inviscid velocity should more accurately be calculated at each lattice link to body surface intersection. Furthermore relatively large inviscid velocities occur at the top and bottom of the cylinder which must be corrected by the perturbation solution (Fig. 7c,d), which further increases numerical errors and slows down the convergence of the hybrid pLBM simulation. Nevertheless, given a fine enough grid $(\Delta x / D=1 / 350)$, accurate results are also achieved in the pLBM model.

This is further confirmed in Table 3, which compares the present LBM and hybrid pLBM results to previously published work. Specifically, the Table lists the experimental results of Wieselsberger [53], the LBM results using a volumetric approach and a no-slip cylinder boundary of $\mathrm{Li}[36]$, results of a fourth-order in space and second-order in time finite difference method by Stalberg [49], and the 2D and 3D finite volume results of Rajani [46]. Overall, both of the present solutions agree well with these results. The converged hybrid pLBM results are in excellent agreement with the LBM results in terms of $C_{L}^{\prime}$ and St, while predicting $0.45 \%$ more drag, likely due to inaccuracies introduced in specifying the body boundary condition discussed earlier. The $C_{D}$ value computed with the hybrid pLBM model, however, falls well within the spread of $C_{D}$ results reported in the Table. 


\begin{tabular}{cccc}
\hline Author(s) (year) & $C_{D}$ & $C_{L}^{\prime}$ & $\mathrm{St}$ \\
\hline Wieselsberger (1932) & 1.34 & - & - \\
Stålberg et al. (2006) & 1.32 & 0.233 & 0.166 \\
Li et al. (2009) & 1.336 & - & 0.164 \\
Rajani et al. (2D) (2009) & 1.3353 & 0.1792 & 0.1569 \\
Rajani et al. (3D) (2009) & 1.3349 & 0.1802 & 0.1569 \\
present LBM & 1.333 & 0.232 & 0.164 \\
present hybrid LBM & 1.339 & 0.232 & 0.164 \\
\hline
\end{tabular}

Table 3: Comparison of flow quantities with earlier results, for a viscous flow past a circular cylinder of diameter $D$.

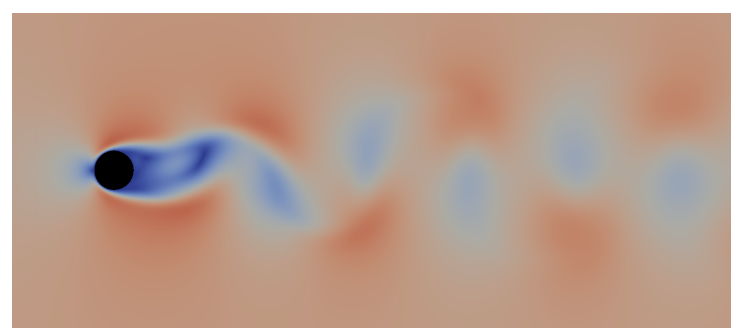

(a) Standard LBM

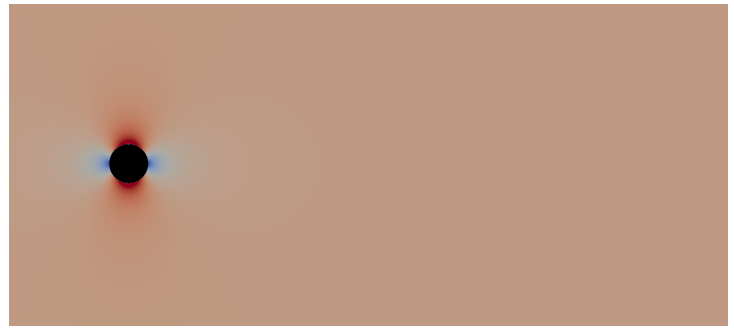

(c) Inviscid velocity $u_{i}^{I}$

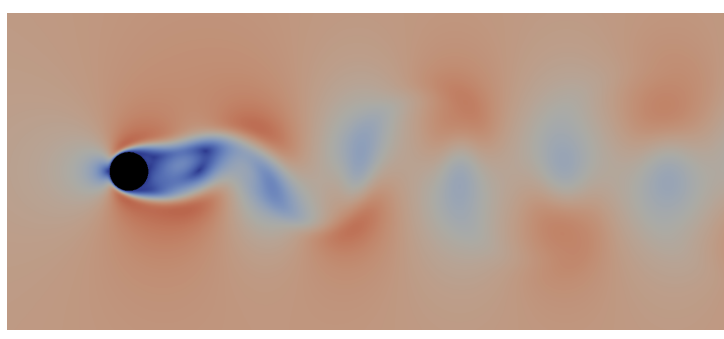

(b) Hybrid LBM $u_{i}^{I}+u_{i}^{P}$

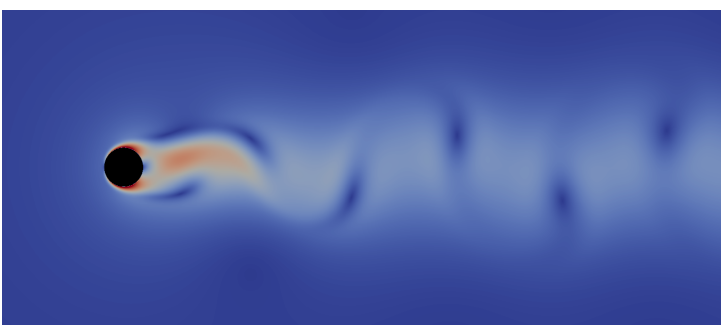

(d) Perturbation velocity $u_{i}^{P}$

0

Velocity Magnitude $(\mathrm{u} / \mathrm{U})$

1.625

Figure 7: Instantaneous velocity magnitude normalized by the flow free-stream velocity $U$ around a cylinder of diameter $D$, at $\operatorname{Re}=100$ during steady state vortex shedding. Total velocity calculated with the LBM (a) and hybrid pLBM (b) are compared at a similar phase of the steady state vortex shedding cycle. The Inviscid velocity $u_{i}^{I}$ driving the hybrid LBM solution (c) and perturbation velocity $u_{i}^{P}(\mathrm{~d})$ are shown, representing the decomposed components of the hybrid pLBM model (b). 


\subsection{Conclusions}

In this paper we show a rigorous validation of the fundamentals of the hybrid LBM approach, choosing to build from simple tests in order to isolate and identify sources of error as simulation complexity is increased. While these applications validate the accuracy, consistency, and convergence of the hybrid LBM approach these simple tests are unable to show the benefits of the hybrid approach. In subsequent papers we will extend the hybrid approach to model turbulent flows around curved bodies using a hybrid LES scheme and turbulent wall model. The latter of which is a novel approach to LBM wall modeling which is then extended to the hybrid LBM. These are validated first for a turbulent channel simulation then extended to capture lift and drag in a high Reynolds number foil validation. We then show that the perturbation LBM component can be used to capture lift on the foil, replacing the typical Kutta condition that is applied in potential flow solutions. This demonstration shows the method's potential as an engineering tool and is particularly useful during scenarios when a potential flow solution alone begins to fail such as large angle of attacks or when 3D effects become important. Furthermor with the hybrid approach this can be done using a significantly reduced numerical domain relative to traditional NS solvers alone, reducing the overall computational requirements.

As simulation complexity is increased further, through the introduction of a free surface, wave structure interaction and wave breaking, traditional NS solvers are often too computationally demanding to be useful. We believe that a our hybrid method may allow for the efficient investigation of problems such as this through the addition of a hybrid volume of fluid method that tracks an overall fluid volume flux as a combination of inviscid and perturbation components. This may allow the simulation of wave breaking in the perturbation solution while suppressing wave breaking in the FNPF solution through a combined free surface condition. This hybrid VOF methodology is under development and may further demonstrate the benefits of the hybrid method.

\subsection{Acknowledgments}

C.M. OReilly, and S.T. Grilli gratefully acknowledge support for this work from grants N000141310687 and N000141612970 of the Office of Naval Research (PM Kelly Cooper). As well as the support of XSEDE grant ENG170010 for GPU computational resources. 


\section{Bibliography}

[1] Alessandrini, B. (2007). Thèse d'Habilitation en Vue de Diriger les Recherches. Ecole Centrale de Nantes, Nantes.

[2] Banari A., Janssen C., Grilli S.T. and M. Krafczyk (2014). Efficient GPGPU implementation of a Lattice Boltzmann Model for multiphase flows with high density ratios. Computers and Fluids, 93:1-17.

[3] Banari A., Janssen C.F., and Grilli S.T. (2014). An efficient lattice Boltzmann multiphase model for 3D flows with large density ratios at high Reynolds numbers. Computers and Mathematics with Applications, 68:1819-1843.

[4] Banari A., Mauzole Y., Hara T., Grilli S.T. and C.F. Janssen (2015). The simulation of turbulent particle-laden channel flow by the Lattice Boltzmann method. International Journal for Numerical Methods in Fluids, 79(10):491-513.

[5] Bhatnagar, P. L., Gross, E. P., and Krook, M. (1954). A model for collision processes in gases. i. small amplitude processes in charged and neutral one-component systems. Phys. Rev., 94(3):511-525.

[6] Biausser B., S.T. Grilli, Fraunié P. and Marcer, R. (2004). Numerical analysis of the internal kinematics and dynamics of three-dimensional breaking waves on slopes. International Journal of Offshore and Polar Engineering, 14(4):247-256.

[7] Bouzidi, M., Firdaouss, M., and Lallemand, P. (2001). Momentum transfer of a LatticeBoltzmann fluid with boundaries. Physics of Fluids, 13:3452-3459.

[8] Brent, R. P. (1973). Algorithms for Minimization Without Derivatives. Prentice Hall. Reviewed in: American Scientist 61 (May-June 1973), 374; Mathematical Programming 4 (1973), 349; Computer J. 16 (1973), 314; Math. Comp. 28 (1974), 865-866.

[9] Dean, R. G. and Dalrymple, R. A. (1991). Water wave mechanics for engineers and scientists (Vol. 2). World Scientific Publishing Company. 
[10] d'Humieres, D., Ginzburg, I., Krafczyk, M., Lallemand, P., and Luo, L.-S. (2002). Multiple relaxation-time Lattice Boltzmann models in three-dimensions. Royal Society of London Philosophical Transactions Series A, 360:437-451.

[11] Freudiger, S., Hegewald, J., and Krafczyk, M. (2008). A parallelization concept for a multiphysics lattice Boltzmann solver based on hierarchical grids. Progress in Computational Fluid Dynamics, 8(1-4):168-178.

[12] Frisch, U., d'Humières, D., Hasslacher, B., Lallemand, P., Pomeau, Y., and Rivet, J.-P. (1987). Lattice Gas Hydrodynamics in Two and Three Dimensions. J. Complex Syst., 1:75136.

[13] Geller, S., Krafczyk, M., Tölke, J., Turek, S., and Hron, J. (2006). Benchmark computations based on Lattice-Boltzmann, Finite Element and Finite volume Methods for laminar Flows. Comp. and Fluids, 35:888-897.

[14] Ginzburg, I. and D'Humieres, D. (2003). Multi-reflection boundary conditions for lattice Boltzmann models. Physical Review E, 68(6):066614.1-066614.30.

[15] Grilli, S.T. (2008) On the development and application of hybrid numerical models in nonlinear free surface hydrodynamics. Keynote lecture in Proc. 8th Intl. Conf. on Hydrodynamics (Nantes, France, 9/08) (P. Ferrant and X.B. Chen, eds.), pps. 21-50.

[16] Grilli, S.T., Dias, F., Guyenne, P., Fochesato, C. and F. Enet (2010). Progress in fully nonlinear potential flow modeling of 3D extreme ocean waves. Chapter 3 in Advances in Numerical Simulation of Nonlinear Water Waves (ISBN: 978-981-283-649-6, edited by Q.W. Ma) (Vol. 11 in Series in Advances in Coastal and Ocean Engineering). World Scientific Publishing Co. Pte. Ltd., pps. 75-128.

[17] Grilli, S., Guyenne, P., and Dias, F. (2001). A fully nonlinear model for three-dimensional overturning waves over arbitrary bottom. International Journal for Numerical Methods in Fluids, 35(7):829-867.

[18] Grilli, S.T. and Horrillo, J. (1997). Numerical Generation and Absorption of Fully Nonlinear Periodic Waves. Journal of Engineering Mechanics, 123(10):1,060-1,069.

[19] Grilli, S.T. and Subramanya, R. (1996). Numerical Modeling of Wave Breaking Induced by Fixed or Moving Boundaries. Computational Mechanics, 17(6):374-391. 
[20] Grilli, S.T., Svendsen, I.A. and Subramanya, R. (1997). Breaking criterion and characteristics for solitary waves on slopes. Journal of Waterway Port Coastal and Ocean Engineering, 123(3):102-112.

[21] Gueyffier, D., Li, J., Nadim, A., Scardovelli, R., and Zaleski, S. (1999). Volume-of-fluid interface tracking with smoothed surface stress methods for three-dimensional flows. Journal of Computational Physics, 152(2):423-456.

[22] Guignard, S., Grilli, S.T., Marcer, R. and Rey, V. (1999). Computation of shoaling and breaking waves in nearshore areas by the coupling of BEM and VOF methods. In Proc. 9th Offshore and Polar Engng. Conf. (ISOPE99, Brest, France, May 1999), Vol. III, 304-309.

[23] Guo, Z., Zheng, C., and Shi, B. (2002). Discrete lattice effects on the forcing term in the Lattice Boltzmann method. Physical Review E, 65(4):046308.1-046308.6.

[24] Harris, J. and Grilli, S.T. (2010). A perturbation approach to large-eddy simulation of wave-induced bottom boundary layer flows. Intl. J. Numer. Meth. Fluids, 68, 1,574-1,604

[25] He, X. and Luo, L.-S. (1997). Lattice Boltzmann model for the incompressible Navier-Stokes equation. Journal of Statistical Physics, 88:927-944.

[26] Hirt, C. and Nichols, B. (1981). Volume of fluid method for dynamics of free boundaries. Journal of Computational Physics, 39:201-221.

[27] Janssen, C. and Krafczyk, M. (2010). A lattice Boltzmann approach for free-surface-flow simulations on non-uniform block-structured grids. Computers and Mathematics with Applications, 59 (7):2215-2235.

[28] Janssen, C.F. (2010) Kinetic approaches for the simulation of non-linear free surface flow problems in civil and environmental engng. $\mathrm{PhD}$ thesis, Technical Univ. Braunschweig.

[29] Janssen C.F., S.T. Grilli and M. Krafczyk (2010) Modeling of Wave Breaking and WaveStructure Interactions by Coupling of Fully Nonlinear Potential Flow and Lattice-Boltzmann Models. Proc. 20th Offshore and Polar Engng. Conf. (ISOPE10, Beijing, China, June 20-25, 2010), pps. 686-693. Intl. Society of Offshore and Polar Engng.

[30] Janssen, C.F., S.T. Grilli and M. Krafczyk (2013). On enhanced non-linear free surface flow simulations with a hybrid LBM-VOF approach. Computers and Mathematics with Applications, 65(2):211-229 
[31] Junk, M., Klar, A., and Luo, L.-S. (2005). Asymptotic analysis of the lattice Boltzmann equation. Journal of Computational Physics, 210(2):676-704.

[32] Körner, C., Thies, M., Hofmann, T., Thürey, N., and Rüde, U. (2005). Lattice Boltzmann model for free surface flow for modeling foaming. Journal of Statistical Physics, 121(1-2):179196(18).

[33] Krafczyk, M., Tölke, J., and Luo, L.-S. (2003). Large-eddy simulations with a multiplerelaxation-time LBE model. Int. J. Mod. Phys. B, 17:33-39.

[34] Lallemand, P. and Luo, L.-S. (2000). Theory of the lattice Boltzmann method: Dispersion, dissipation, isotropy, galilean invariance, and stability. Physical Review, E 61:6546-6562.

[35] Latt, J. (2007). Hydrodynamic limit of lattice Boltzmann equations. PhD thesis, Univ. Genéve

[36] Li, Y., Zhang, R., Shock, R., and Chrn, H. (2009). Prediction of vortex shedding from a circular cylinder using a volumetric Lattice-Boltzmann boundary approach. The European Physical Journal Special Topics, 171, 91-97.

[37] Longuet-Higgins, M.S. (1953). Mass transport in water waves. Phil. Trans. Royal Soc. London, A, 535-581.

[38] Martin, J. and Moyce, W. (1952). An experimental study of the collapse of liquid columns on a rigid horizontal plane. Technical report.

[39] Mei, R., Luo, L.-S., Shyy, W. (1999). An Accurate Curved Boundary Treatment in the Lattice Boltzmann Method. Journal of Computational Physics, 155(2):307-330.

[40] Mei, R., Luo, L.-S., Lallemand, P., and d'Humieres, D. (2006). Consistent initial conditions for lattice boltzmann simulations. Computers and Fluids, 35(8-9):855-862.

[41] O'Reilly, C., Grilli, S.T., Dahl, J.M., Banari, A., Janssen, C.F., Shock, J.J. and M. Uberrueck. (2015) Solution of viscous flows in a hybrid naval hydrodynamic scheme based on an efficient Lattice Boltzmann Method. Proc. 13th Intl. Conf. on Fast Sea Transportation (FAST 2015; Washington D.C., September 1-4, 2015), 9 pps., https://personal.egr.uri.edu/grilli/OReilly-etal-FAST2015.pdf. 
[42] O'Reilly C.M., Grilli S.T., Harris J.C., Mivehchi A., Janssen C.F. and J. Dahl (2016). Development of a hybrid LBM-potential flow model for Naval Hydrodynamics. Proc. 15th Journée de l'hydrodynamique (JH2016) (November 22-24, Brest, France), 15 pps., http://website.ecnantes.fr/actesjh/images/15JH/Articles/oreilly.pdf.

[43] O'Reilly C.M., S.T. Grilli, J.C. Harris, A. Mivehchi, C.F. Janssen and J.M. Dahl (2017). A Hybrid Solver Based on Efficient BEM-potential and LBM-NS Models: Recent LBM Developments and Applications to Naval Hydrodynamics. In Proc. 27th Offshore and Polar Engng. Conf. (ISOPE17, San Francsico, USA. June 2017), Intl. Society of Offshore and Polar Engng., pps. 713-720, https://personal.egr.uri.edu/grilli/OReillyetal_ISOPE17.pdf.

[44] Parker, B. and Youngs, D. (1992). Two and three dimensional eulerian simulation of fluid flow with material interfaces. Technical report, UK Atomic Weapons Establishment.

[45] Quian, Y. H., d'Humieres, D., and Lallemand, P. (1992). Lattice BGK models for Navier Stokes equations. Europhysics Letters, 17:479-484.

[46] Rajani, B., Kandasamy, A., and Majumdar, S. (2009). Numerical simulation of laminar flow past a circular cylinder. Applied Mathematical Modelling, 33, 1228-1247.

[47] Reliquet, G., A. Drouet, P.-E. Guillerm, L. Gentaz and P. Ferrant (2014). Simulation of wave-ship in ction in regular and irregular seas under viscous flow theory using the SWENSE method. Proc. 30th Symp. Naval Hydrod., (Tasmania, 11/2014), 11 pps.

[48] Salih, A. and Moulic, S. G. (2006). A level set formulation for the numerical simulation of impact of surge fronts. SADHANA - Academy Proceedings in Engineering Sciences, 31:697707.

[49] Stålberg, E., Bruger, A., Lotstedt, P., Johansson, A., and Henningson, D. (2006). High Order Accurate Solution of Flow Past a Circular Cylinder. Journal of Scientific Computing, Vol 27.

[50] Stokes G.G. (1851). On the effect of the internal friction of fluids on the motion of pendulums. Cambridge Philosophical Transactions; 9:8-106.

[51] Tölke, J.(2008). Implementation of a lattice Boltzmann kernel using the compute unified device architecture developed by NVIDIA. Computing and Visualization in Science, 1:29-39. 
[52] Tölke, J. and Krafczyk, M. (2008b). Teraflop computing on a desktop PC with GPUS for 3D CFD. International Journal of Computational Fluid Dynamics, 22:443-456.

[53] Wieselsberger, C. (1932). New data on the laws of fluid resistance. Technical Notes. National Advisory Committee for Aeronautics, No. 84.

[54] Youngs, D. (1982). Time-dependent multimaterial flow with large fluid distortion. In Morton, K. and Baines, M., editors, Numerical Methods for Fluid Dynamics, pages 273-285. Academic Press. 


\section{CHAPTER 2}

A hybrid lattice-Boltzmann method and potential flow solver applied to high Reynolds number incompressible flows

Christopher M. O’Reilly ${ }^{a, b}$, Stephan T. Grilli ${ }^{a}$, Christian F. Janßen $^{c}$, Jason M. Dahl ${ }^{a}$, Jeffry C. Harris ${ }^{d}$

${ }^{a}$ Department of Ocean Engineering, University of Rhode Island, Narragansett, RI, USA

${ }^{b}$ Navatek Ltd., South Kingstown, RI, USA

${ }^{c}$ Institute for Fluid Dynamics and Ship Theory, Hamburg University of Technology, Hamburg, Germany

${ }^{d}$ Institut de Recherche sur les Phenomenes Hors Equilibre (IRPHE), Marseille, France

This manuscript will be submitted for review to the Journal of Computational Physics. 


\section{Abstract}

We report on the development and validation of a 3D hybrid numerical model for the simulation of complex wave-structure interactions that is based on a perturbation method, in which the velocity and pressure are expressed as the sum of an inviscid flow with a viscous perturbation. The far- to near-field flow can be solved with a Boundary Element Method (BEM), based on fully nonlinear potential flow theory, and the near-field perturbation flow is solved with a NavierStokes (NS) model based on a Lattice Boltzmann Method (LBM) with a Large Eddy Simulation (LES) of the turbulence. We summarize the hybrid model formulation, where the viscous perturbation flow is modeled using a modified LBM collision operator, resulting in a perturbation LBM (pLBM). The pLBM is then extended for the simulation of turbulence using the LES and a wall model for the viscous/turbulent sub-layer near solid boundaries. The latter is based on a novel LBM wall model that is generalized for an arbitrary geometry, which is first presented for the LBM then extended to the pLBM. The model is validated by simulating turbulent flows over a flat plate for $\operatorname{Re} \in\left[3.7 \times 10^{4} ; 1.2 \times 10^{6}\right]$, for which the friction coefficient computed on the plate and turbulent properties agree well with experiments and direct NS simulations. We then simulate the flow past a NACA0012 foil using the LBM-LES and pLBM-LES with the wall model, for $\operatorname{Re}=1.44 \times 10^{6}$, and show a good agreement of lift forces, drag forces and pressure distribution with experiments and other numerical methods. Results obtained with the hybrid LBM model are either nearly identical or improved relative to those of the standard LBM, but for a smaller computational domain, demonstrating the benefits of the hybrid approach.

\subsection{Introduction}

Numerical models simulating the irrotational motion of an incompressible, inviscid fluid, based on potential flow theory, are computationally efficient and sufficiently accurate to simulate many engineering fluid problems, such as those involving free surface waves and wavestructure interactions (e.g., [Grilli, 2010]). However, potential flow models cannot be used in applications where viscous effects are important, for instance, in the boundary layer near solid boundaries, in the wake of bluff bodies, or to simulate surface wave breaking. Standard Computational Fluid Mechanics (CFD) Navier-Stokes (NS) solvers, such as based on a finite volume (e.g., [Hirt and Nichols, 1981]) or Lattice Boltzmann (LBM) method (e.g., [He and Luo, 1997, d'Humieres et al., 2002, Krafczyk et al., 2003, Geller et al., 2006, Janssen and Krafczyk, 2009, Janssen, 2010]), can model these types of flows, but can be computationally costly. Additionally, 
for free surface flows, NS solvers are often too numerically dissipative to model wave propagation over long distances [Biausser et al., 2004].

To more efficiently solve a broad class of hydrodynamics problems of interest to many engineering disciplines, in this work, we detail the development of a high-fidelity but low cost hybrid numerical model, that combines potential flow and NS models, and applies each model in the region where it is most effective. This hybrid model is based on a perturbation method proposed in earlier work, but for different numerical methods and problems [Alessandrini, 2007, Grilli, 2008]. For instance, it was successfully used to model turbulent flows, using a finite volume method, and validated for turbulent channel and wave induced boundary layer flows [Harris and Grilli, 2012] and for linear ship seakeeping ([Reliquet et al, 2014]). Unlike one- or two-way coupled models applied over separate regions of the computational domain (e.g., [Biausser et al., 2004, Guignard et al., 1999]), in this method, both the velocity and pressure fields are expressed as the sum of inviscid/irrotational $(I)$ and viscous perturbation $(P)$ components, each solved using different numerical models in separate but overlapping computational domains. This method is sometimes referred to in fluid mechanics as the Helmholtz decomposition. More specifically, the $I$ fields are solved with a potential flow model typically over a larger size domain extending to the far-field, whereas the $P$ fields are solved based on a modified (perturbed) NS equation, here with a LBM model, in a smaller near-field domain in which viscous effects are deemed important based on the considered problem (this will be made more clear later). Thus, the more computationally demanding pLBM model is only used in the smaller near-field domain where viscous/turbulent effects matter, with its solution forced by results of the potential flow model applied to the larger domain. Hence this hybrid approach is much more computationally efficient than applying a LBM model to the entire domain, while ensuring that the complete NS solution is solved where the physics calls for it.

In engineering applications involving complex boundary conditions and/or boundary/structure geometry, the model solving potential flow equations over the entire computational domain must itself be an optimized generic numerical solver, such as based on the higher-order Boundary Element Method, and feature fully nonlinear free surface boundary conditions if applicable [Janssen et al., 2010, Harris and Grilli, 2012]. Such cases, however, are not considered here but are described in recent work [O'Reilly et al., 2018]. The present paper instead concentrates on detailing the development of the pLBM model and validating it on a series of applications for which there are analytical solutions of the potential flow fields $I$ that can be used in the hybrid 
model to force the pLBM solution. This was first done for low Reynolds number applications in [Janssen et al., 2018] and here we develop LES and wall boundary turbulence models for the pLBM and extend our validation suite into the turbulent regime, which will enable the method to be used in high Reynolds number applications.

In our work, we use a LBM to solve NS equations, instead of a finite volume solver, in part because the data locality and kernel simplicity of the LBM allow for a very efficient parallel implementation of the model on a "General Purpose Graphical Processor Units" (GPGPU) [Janssen and Krafczyk, 2010, Tölke, 2008, Tölke and Krafczyk, 2008b]. While a single GPGPU still has a limited memory, a multi-GPGPU implementation of the LBM may achieve a higher computational efficiency, for an identical accuracy, than traditional CFD solvers implemented on a massively parallel CPU cluster. In the hybrid method context, for many engineering applications, the reduced-size pLBM computational domain can often be simulated using a single GPGPU [O'Reilly et al., 2017], allowing simulations to be run on a desktop computer equipped with a relatively inexpensive GPGPU co-processor. When the potential flow is also solved with a numerical model, e.g., BEM based, its solution may then be calculated using the computer's often parallelized CPUs, with limited conflicting resource requirements. If a traditional NS solver were to be used in place of the LBM, a significant number of CPU nodes would be required to run it at an accuracy equivalent to that of the LBM, leading to competing computational resources when combined with the potential flow solver.

The coupling between continuum mechanics-based equations (or models), such as potential flow, and the kinetic-based LBM is less straightforward than earlier implementations of the hybrid method based on a volume of fluid NS solver [Harris and Grilli, 2012]. In particular, one must derive a pLBM equivalent to the nonlinear $I-P$ coupling terms that appear in the perturbation NS equations, as described in the following sections. To assess the ability of the LBM to simulate strongly nonlinear free surface flows, Janssen et al. [Janssen, 2010, Janssen et al., 2010, Janssen and Krafczyk, 2010] simulated the two-dimensional (2D) "weak coupling" wave breaking results reported in earlier work [Biausser et al., 2004, Guignard et al., 1999], using a LBM in combination with a Volume Of Fluid (VOF) interface tracking method. In such cases, the LBM model was simply initialized with potential flow results for waves that had been propagated up to the breaking point in a potential flow BEM model [Grilli and Horrillo, 1997, Grilli and Subramanya, 1996, Grilli et al., 1997]. Next, the same authors computed similar results with the hybrid method, in which the $I-P$ coupling terms were 
represented as LBM body force terms, using the pre-computed $I$ fields to force the $P$ field solution through these terms. This approach, while proven effective, required computing spatial derivatives of both the $I$ and $P$ fields using finite difference approximations that yielded a compact but non-local LBM kernel. Additional analyses showed that this approach caused higher truncation errors in the pLBM than in the original LBM collision operator and reduced the overall efficiency of the parallelized GPGPU solution. Therefore, Janssen [Janssen, 2010] suggested instead to introduce the nonlinear $I-P$ coupling terms directly into the LBM equilibrium probability distribution functions (EPDFs), hence, to develop perturbation EPDFs or pEPDFs. The latter were incrementally developed, implemented, and validated as part of the development of a pLBM model component to a hybrid naval hydrodynamic solver, in which the potential flow solution, with fully nonlinear free surface boundary conditions (FNPF), was computed using a higher-order BEM model [O'Reilly et al., 2015, O'Reilly et al., 2016, O'Reilly et al., 2017, Janssen et al., 2018].

In this paper, we focus on the development and validation of the pLBM solver applied to the modeling of turbulent flows after first describing the pLBM formulation with a Multiple Relaxation Time (MRT) collision operator. To model the sub-grid turbulence within the bulk of the fluid, the LBM-LES model originally proposed by [Krafczyk et al., 2003] is adapted to consider the influence of the hybrid coupling. For many applications, an additional "wall model" is required for a more accurate representation of turbulent boundary layers near solid boundaries without the need for a refined discretization. Here we present a wall model approach to LBM that is based on the work of [Malaspinas and Sagaut, 2014] where modifications are proposed that extend its validity to allow a more accurate simulation of wall boundaries of arbitrary shape and orientation. The wall model is then extended for the pLBM. The LBM-LES with the wall model and its pLBM counterpart are validated by first simulating the flow in turbulent channels using the test case first presented in [Malaspinas and Sagaut, 2014], but our modifications allow for model convergence, and a nominal difference between the LBM and pLBM results are observed. Next, the method is validated in a more rigorous test, by computing the drag, lift, and pressure distribution on a NACA0012 foil at a Reynolds number $\mathrm{Re}=1.44 \times 10^{6}$ using both the standard LBM and perturbation LBM.

\subsection{The Lattice Boltzmann Method}

In the LBM, the macroscopic NS equations are modeled by solving an equivalent mesoscopic problem in which the fluid is represented by particles interacting over a (typically regular) lattice 
(or grid), through their distribution functions (DF) $f(t, \mathbf{x}, \xi)$, representing the normalized probability to find a particle at location $\mathbf{x}$ at time $t$ with velocity $\xi$; the macroscopic hydrodynamic quantities (e.g., velocity, pressure,...) are defined as moments of the DFs.

\subsubsection{LBM basics}

The time evolution of discrete particle DFs is governed by the Boltzmann advection-collision equation,

$$
\frac{D f_{\alpha}}{D t}=\frac{\partial f_{\alpha}(t, \mathbf{x})}{\partial t}+\mathbf{e}_{\alpha} \cdot \frac{\partial f_{\alpha}(t, \mathbf{x})}{\partial \mathbf{x}}=\Omega_{\alpha}+\mathbf{B}_{\alpha}
$$

in which $\mathbf{e}_{\alpha}$ denotes discrete particle velocities, $\Omega_{\alpha}$ is a collision operator describing interactions between particles, and $B_{\alpha}$ represents volume forces such gravity. Eq. (57) is discretized over a regular lattice, of grid spacing $\Delta x$ using $n=19$ discrete particle velocities (standard D3Q19 scheme), which point in the directions of 18 neighboring particles from a given particle location; thus: $\mathbf{e}_{\alpha}=\{0,0,0\} ;\{ \pm c, 0,0\} ;\{0, \pm c, 0\} ;\{0,0, \pm c\} ;\{ \pm c, \pm c, 0\} ;\{ \pm c, 0, \pm c\} ;\{0, \pm c, \pm c\}$, for $\alpha=$ $0, \ldots, 18$. With this choice of lattice, isotropy is maintained with lattice dependent directional weights $w_{\alpha}$ are, $w_{0}=1 / 3, w_{1 \ldots 6}=1 / 18$ and $w_{7 \ldots 18}=1 / 36$ (see Eq. (59)) and the relation $c_{s}=c / \sqrt{3}$ for the speed of sound $c_{s}$ and particle propagation speed $c$ is found.

In the standard single relaxation time (SRT) LBM, Eq. (2) is discretized by finite differences in space and time as,

$$
f_{\alpha}\left(t+\Delta t, \mathbf{x}+\mathbf{e}_{\alpha} \Delta t\right)-f_{\alpha}(t, \mathbf{x})=-\frac{\Delta t}{\tau}\left\{f_{\alpha}(\mathbf{x}, t)-f_{\alpha}^{e q}(\rho, \mathbf{u})\right\}+B_{\alpha}^{\prime}
$$

where $f_{\alpha}^{e q}(\rho, \mathbf{u})$ are equilibrium DFs, functions of the macroscopic fluid density $\rho$ and velocity $\mathbf{u}, \Delta t$ is time step (with $c=\Delta x / \Delta t$ ), and $\tau=3 \nu / c^{2}+\Delta t / 2$, a nondimensional relaxation time (SRT) expressed as a function of fluid viscosity $\nu$. LBM simulations are typically split up into a nonlinear collision step, which locally drives the particle DFs to equilibrium, and a linear propagation step, during which the evolved DFs are advected.

For the LBM solution to satisfy the incompressible NS equations [He and Luo, 1997], the following equilibrium function is chosen,

$$
f_{\alpha}^{e q}(\rho, \mathbf{u})=w_{\alpha}\left(\rho+\rho_{o}\left(3 \frac{\left(\mathbf{u} \cdot \mathbf{e}_{\alpha}\right)}{c^{2}}+\frac{9}{2} \frac{\left(\mathbf{u} \cdot \mathbf{e}_{\alpha}\right)^{2}}{c^{4}}-\frac{3}{2} \frac{\mathbf{u}^{2}}{c^{2}}\right)\right)
$$

where a Chapman-Enskog expansion (described in the appendix) to Eq. (58) shows that the incompressible NS equations are recovered up to $\mathcal{O}\left(\Delta x^{2}\right)$ and $\mathcal{O}\left(\mathrm{Ma}^{2}\right)$ errors, with $\mathrm{Ma}=U / c_{s}$, the Mach number and $U$, a characteristic flow velocity. Variables $\rho_{o}$ and $\rho$ represent the average fluid 
density and a small perturbation from that density, respectively. The hydrodynamic quantities are found as low order moments of the DFs,

$$
\rho=\sum_{\alpha=1}^{n} f_{\alpha}, \quad \rho_{o} u_{i}=\sum_{\alpha=1}^{n} e_{\alpha i} f_{\alpha}
$$

d'Humieres et al. (2002) showed that more accurate and stable results can be obtained, particularly for high Reynolds numbers, using the multiple relaxation time (MRT) LBM. This method incorporates higher-order moments (i.e., hydrodynamic quantities and their fluxes) into the solution, which have important physical significance [Lallemand and Luo, 2000] and are useful to implement LES in the LBM for turbulent flows. In the MRT, the collision operator in the right hand side of Eq. (58) is replaced by the following $(\beta=0, \ldots, 18 ; \gamma, \delta=0, \ldots, 15$; repeated indices in equations mean an implicit summation),

$$
\Omega_{\alpha}=-M_{\alpha \gamma}^{-1} S_{\gamma \delta}\left(M_{\beta \delta} f_{\beta}-m_{\delta}^{e q}\right)
$$

where $M_{\alpha \gamma}$ is the transformation matrix from DFs to moments, with $f_{\alpha}=M_{\alpha \gamma}^{-1} m_{\gamma}$ and $S_{\gamma \delta}$ is a diagonal collision matrix of relaxation parameters, weighing different properties of the fluid. Equilibrium moments $m_{\gamma}^{e q}$ are derived from Eq. (59) as,

$$
\begin{array}{r}
m_{0}^{e q}=\rho, \quad m_{3}^{e q}=\rho u_{x}, \quad m_{5}^{e q}=\rho u_{y}, \quad m_{7}^{e q}=\rho u_{z} \\
m_{1}^{e q}=e^{e q}=\rho_{0}\left(u_{x}^{2}+u_{y}^{2}+u_{z}^{2}\right), \quad m_{9}^{e q}=3 p_{x x}^{e q}=\rho_{0}\left(2 u_{x}^{2}-u_{y}^{2}-u_{z}^{2}\right) \\
m_{11}^{e q}=p_{z z}^{e q}=\rho_{0}\left(u_{y}^{2}-u_{z}^{2}\right), \quad m_{13}^{e q}=p_{x y}^{e q}=\rho_{0}\left(u_{x} u_{y}\right) \\
m_{14}^{e q}=p_{y z}^{e q}=\rho_{0}\left(u_{y} u_{z}\right), \quad m_{15}^{e q}=p_{x z}^{e q}=\rho_{0}\left(u_{x} u_{z}\right)
\end{array}
$$

We now consider the scaling of our physical variables to non-dimensional lattice variables (denoted by prime variables in the following) using spatial, temporal, and mass scales $\lambda, \tau$, and $\varpi$, respectively. For numerical efficiency a mesh Courant number of $C_{o}=1$ is chosen to remove the need for finite differencing in Eq. (58). In LBM, one typically assumes, $\Delta x^{\prime}=\Delta x / \lambda=1$, $\Delta t^{\prime}=\Delta t / \tau=1, c^{\prime}=c \tau / \lambda=1$, and $m^{\prime}=m / \varpi=1$.

Accordingly, our physical variables are scaled based on the flow Mach number Ma $=u / c_{s}=$ $u^{\prime} / c_{s}^{\prime}$ and Reynolds number, $\operatorname{Re}=u \ell / \nu=u^{\prime} \ell^{\prime} / \nu^{\prime}$ (with $\ell$ a representative length scale of the flow) and our physical length scale, $\lambda$, becomes $\lambda=\Delta x / \ell$. Inserting $c_{s}^{\prime}=c^{\prime} / \sqrt{3}=1 / \sqrt{3}$ into the Mach number equation gives $u^{\prime}=\operatorname{Ma} \sqrt{3}$ and $\tau$ is found as $\tau=\lambda u / u^{\prime}$. Hence, the non-dimensional fluid viscosity reads, $\nu^{\prime}=\nu \tau / \lambda^{2}$. For simplicity, in the following, we will drop the prime notation for non-dimensional variables unless stated otherwise. 


\subsubsection{Equations for the perturbation LBM}

Here, we first recap the expressions of the NS perturbation method ([Grilli, 2008]; [Harris and Grilli, 2012]) and develop the corresponding LBM equations with MRT. Applying a Helmholtz decomposition to the flow, both the velocity and pressure are expressed as,

$$
u_{i}=u_{i}^{I}+u_{i}^{P} \quad \text { with } \quad \tilde{p}=\tilde{p}^{I}+\tilde{p}^{P}
$$

where $\tilde{p}=p+\rho g x_{3}-\frac{2}{3} \rho k$ denotes the dynamic pressure, with $k$ the turbulent kinetic energy. As indicated before, superscripts $I$ denote inviscid flow quantities, with $u_{i}^{I}=\nabla_{i} \phi^{I}$ satisfying Euler equations, and superscripts $P$ represents perturbation flow quantities that are driven by the inviscid flow fields. After applying this decomposition and substituting Euler's equations, the perturbation NS equations read,

$$
\begin{aligned}
\frac{\partial u_{i}^{P}}{\partial x_{i}} & =0 \\
\frac{\partial u_{i}^{P}}{\partial t}+u_{j}^{P} \frac{\partial u_{i}^{P}}{\partial x_{j}} & =-\frac{1}{\rho} \frac{\partial \tilde{p}^{P}}{\partial x_{i}}+\left(\nu+\nu_{t}\right) \frac{\partial^{2} u_{i}^{P}}{\partial x_{j} \partial x_{j}}-\left(\frac{\partial u_{i}^{I}}{\partial x_{j}} u_{j}^{P}+u_{j}^{I} \frac{\partial u_{i}^{P}}{\partial x_{j}}\right)+2 \frac{\partial \nu_{t}}{\partial x_{j}} S_{i j}
\end{aligned}
$$

where $\nu$ and $\nu_{t}$ are kinematic molecular and turbulent viscosity, respectively, with the latter being expressed through the Smagorinsky method as,

$$
\nu_{t}=\left(C_{S} \Delta\right)^{2}|\mathbf{S}|, \quad \text { with } \quad S_{i j}=S_{i j}^{P}+S_{i j}^{I}=\frac{1}{2}\left(\frac{\partial u_{i}^{P}}{\partial x_{j}}+\frac{\partial u_{j}^{P}}{\partial x_{i}}+\frac{\partial u_{i}^{I}}{\partial x_{j}}+\frac{\partial u_{j}^{I}}{\partial x_{i}}\right)
$$

where $C_{S}$ is the Smagorinsky constant, $\Delta$ a grid filtering length scale, and $S_{i j}$ the rate of strain

tensor, which here is expressed as the sum of its perturbation $S_{i j}^{P}$ and inviscid $S_{i j}^{I}$ components, both found as a function of the corresponding velocity components.

To recover Eq. (64) in the perturbation LBM, we decompose the DFs into their inviscid and perturbation components, $f_{\alpha}=f_{\alpha}^{I}+f_{\alpha}^{P}$. Introducing this decomposition into in Eq. (58) and subtracting the LBM equation for the inviscid flow, we get,

$$
\left.\left.f_{\alpha}^{P}\left(t+\Delta t, \mathbf{x}+\mathbf{e}_{\alpha} \Delta t\right)\right)-f_{\alpha}^{P}(t, \mathbf{x})\right)=-\frac{\Delta t}{\tau}\left\{f_{\alpha}^{P}(t, \mathbf{x})-f_{\alpha}^{e q}\left(\rho^{I}+\rho^{P}, \mathbf{u}^{I}+\mathbf{u}^{P}\right)+f_{\alpha}^{e q, I}\left(\rho^{I}, \mathbf{u}^{I}\right)\right\}
$$

where the $f_{\alpha}^{e q, I}\left(\rho^{I}, \mathbf{u}^{I}\right)$ can exactly reproduce Euler's equations [Janssen et al., 2018]. The perturbation equilibrium DFs are then found as, $f_{\alpha}^{e q, P}\left(\rho^{P}, \mathbf{u}^{P}, \mathbf{u}^{I}\right)=f_{\alpha}^{e q}\left(\rho^{I}+\rho^{P}, \mathbf{u}^{I}+\mathbf{u}^{P}\right)-$ $f_{\alpha}^{e q, I}\left(\rho^{I}, \mathbf{u}^{I}\right)$

$$
f_{\alpha}^{e q, P}=w_{\alpha}\left(\rho^{P}+\rho_{o}\left(3 \frac{\mathbf{u}^{P} \cdot \mathbf{e}_{\alpha}}{c^{2}}+\frac{9}{2} \frac{\left(\mathbf{e}_{\alpha} \cdot \mathbf{u}^{P}\right)^{2}+2\left(\mathbf{e}_{\alpha} \cdot \mathbf{u}^{P}\right)\left(\mathbf{e}_{\alpha} \cdot \mathbf{u}^{I}\right)}{c^{4}}-\frac{3}{2} \frac{\left(\mathbf{u}^{P}\right)^{2}+2 \mathbf{u}^{P} \cdot \mathbf{u}^{I}}{c^{2}}\right)\right),
$$


which satisfy,

$$
\sum_{\alpha=1}^{n} f_{\alpha}^{e q, P}=\rho^{P}, \quad \sum_{\alpha=1}^{n} e_{\alpha i} f_{\alpha}^{e q, P}=\rho_{o} u_{i}^{P}, \quad \sum_{\alpha=1}^{n} e_{\alpha i} e_{\alpha j} f_{\alpha}^{e q, P}=p^{P} \delta_{i j}+\rho_{o} u_{i}^{I} u_{j}^{P}+\rho_{o} u_{i}^{P} u_{j}^{I}+\rho_{o} u_{i}^{P} u_{j}^{P} .
$$

Extending this formulation to the MRT, assuming a collision operator expressed by Eq. (61), we find the equilibrium moments,

$$
\begin{array}{r}
m_{1}^{e q, P}=e^{e q}=\rho_{0}\left(\left(u_{x}^{P}\right)^{2}+\left(u_{y}^{P}\right)^{2}+\left(u_{z}^{P}\right)^{2}+2 u_{x}^{P} u_{x}^{I}+2 u_{y}^{P} u_{y}^{I}+2 u_{z}^{P} u_{z}^{I}\right) \\
m_{9}^{e q, P}=3 p_{x x}^{e q}=\rho_{0}\left(2\left(u_{x}^{P}\right)^{2}-\left(u_{y}^{P}\right)^{2}-\left(u_{z}^{P}\right)^{2}+4 u_{x}^{P} u_{x}^{I}-2 u_{y}^{P} u_{y}^{I}-2 u_{z}^{P} u_{z}^{I}\right) \\
m_{11}^{e q, P}=p_{z z}^{e q}=\rho_{0}\left(\left(u_{y}^{P}\right)^{2}-\left(u_{z}^{P}\right)^{2}+2 u_{y}^{P} u_{y}^{I}-2 u_{z}^{P} u_{z}^{I}\right), \quad m_{13}^{e q, P}=p_{x y}^{e q}=\rho_{0}\left(u_{x}^{P} u_{y}^{P}+u_{x}^{P} u_{y}^{I}+u_{y}^{P} u_{x}^{I}\right) \\
m_{14}^{e q, P}=p_{y z}^{e q}=\rho_{0}\left(u_{y}^{P} u_{z}^{P}+u_{y}^{P} u_{z}^{I}+u_{z}^{P} u_{y}^{I}\right), \quad m_{15}^{e q, P}=p_{x z}^{e q}=\rho_{0}\left(u_{x}^{P} u_{z}^{P}+u_{x}^{P} u_{z}^{I}+u_{z}^{P} u_{x}^{I}\right)
\end{array}
$$

and moments that are not listed above are unchanged from the standard MRT formulation.

A Chapman-Enskog expansion using Eq. (67) shows that the laminar components of Eq. (64) are recovered [Janssen et al., 2018] (seen in Appendix A). Note the nonlinear presence of interaction terms between the $I$ and $P$ fields in Eq. (67) and Eq. (68), expressing the nonlinear inviscid flow forcing on the perturbation fields without the need for directly evaluating the derivative of the velocity. We now focus on recovering the turbulent components of Eq. (64) using the perturbation LBM.

\subsubsection{LES turbulence modeling with the perturbation LBM}

Krafczyk et al. (2003) expressed the 2nd-order moments of the DFs as,

$$
P_{i j}=\sum_{\alpha=1}^{n} e_{\alpha i} e_{\alpha j} f_{\alpha}=c_{s}^{2} \rho_{o} \delta_{i j}+\rho_{o} u_{i} u_{j}-\frac{2 c_{s}^{2} \rho_{o}}{s_{x x}} S_{i j}
$$

where $s_{2}$ is a relaxation rate for these moments, and showed that they are related to 2 nd-order moments in the MRT, $3 p_{x x}, p_{z z}, p_{x y}, p_{y z}$, and $p_{x z}$. The 1st and 2nd terms in Eq. (70)'s RHS are functions of flow quantities obtained through other zeroth order moments of the DFs, and the rate of strain tensor can be expressed as,

$$
S_{i j}=\frac{s_{x x}}{2 c_{s}^{2} \rho}\left\{c_{s}^{2} \rho \delta_{i j}+\rho u_{i} u_{j}-P_{i j}\right\}=\frac{s_{x x}}{2 c_{s}^{2} \rho_{o}} Q_{i j} .
$$

Krafczyk et al. (2003) assumed that the $Q_{i j}$ 's are functions of the non-equilibrium part of the DFs, $f_{\alpha}^{n e q}=f_{\alpha}-f_{\alpha}^{e q}$ and provided their expressions as a function of the 2nd-order MRT moments. Similar to the LES Eq. (65), they then calculated the turbulent viscosity as,

$$
\nu_{t}=\left(C_{S} \Delta\right)^{2}|\mathbf{S}|=\frac{s_{x x}}{2 c_{s}^{2} \rho}\left(C_{s} \Delta\right)^{2}|\mathbf{Q}|, \quad \text { with } \quad|\mathbf{Q}|=\sqrt{Q_{i j} Q_{i j}}
$$


and expressed the relaxation rate of the 2nd-order moments as,

$$
s_{x x}=\frac{1}{\tau_{\text {total }}}=\frac{1}{\tau_{0}+\tau_{t}} \quad \text { with } \quad \tau_{t}=\frac{1}{2}\left(\sqrt{\tau_{0}^{2}+18\left(C_{s} \Delta\right)^{2}|\mathbf{Q}|}-\tau_{0}\right)
$$

where $\tau_{0}$ is the relaxation time based on the molecular viscosity.

When applying the LES to the pLBM, the moments $P_{i j}^{P}$ are given by the last Eq. (68), yielding an expression for the perturbation rate of strain tensor that features nonlinear interaction terms between the $I$ and $P$ fields,

$$
S_{i j}^{P}=\frac{s_{x x}}{2 c_{s}^{2} \rho}\left(c_{s}^{2} \rho \delta_{i j}+\rho_{o} u_{i}^{P} u_{j}^{P}+\rho_{o} u_{i}^{I} u_{j}^{P}+\rho_{o} u_{i}^{P} u_{j}^{I}-P_{i j}^{P}\right)=\frac{s_{x x}}{2 c_{s}^{2} \rho} Q_{i j}^{P} .
$$

The rate of strain tensor for the total flow is thus given by,

$$
S_{i j}=\frac{s_{x x}}{2 c_{s}^{2} \rho} Q_{i j}^{P}+S_{i j}^{I}
$$

Therefore the $|\mathbf{Q}|$ term to use in LES Eq. (72) and Eq. (73) in combination with the MRT LBM Eqs. (66) to (69), is modified as follows,

$$
|\mathbf{Q}|=\sqrt{R_{i j} R_{i j}} \quad \text { with } \quad R_{i j}=Q_{i j}^{P}+\frac{2 c_{s}^{2} \rho_{o}}{s_{x x}} S_{i j}^{I}
$$

where the $Q_{i j}^{P}$ terms are computed with Eq. (74). Finally, considering Eq. (73) in Eq. (105) found from the Chapman-Enskog expansion (seen in Appendix A) gives

$$
\frac{\partial u_{i}^{P}}{\partial t}+u_{j}^{P} \frac{\partial u_{i}^{P}}{\partial x_{j}}=-\frac{1}{\rho} \frac{\partial \tilde{p}^{P}}{\partial x_{i}}+\left(\nu+\nu_{t}\right) \frac{\partial^{2} u_{i}^{P}}{\partial x_{j} \partial x_{j}}-\left(\frac{\partial u_{i}^{I}}{\partial x_{j}} u_{j}^{P}+u_{j}^{I} \frac{\partial u_{i}^{P}}{\partial x_{j}}\right) .
$$

Note that the final term related to the gradient of the turbulent viscosity in Eq. (64), $2 \frac{\partial \nu_{t}}{\partial x_{j}} S_{i j}$, is not recovered with the approach described here. It will exist both with and without the perturbation decomposition of the NS-LES equations and the standard LBM-LES scheme discussed here also does not recover this term. The authors are unaware of a LBM-LES method that is able to do so and it is possible to resolve it by using an additional body force term in Eq. (58). However, the results presented here show a good agreement with their reference solutions, so it is assumed that this term is small and further investigation is required.

\subsection{Turbulent Wall Model}

Typical naval hydrodynamics flows are fully turbulent, with $\operatorname{Re}>10^{6}$. Thus, the turbulent boundary layers (BL) near solid boundaries (e.g., ship hull) must be properly modeled in the LBM. Since resolving the BL in the LBM grid would be computationally prohibitive (even with grid refinement), besides the LES of the flow, this requires implementing a turbulent a wall model to properly capture velocity gradients near the wall and resulting wall stresses. 
Below, we describe an extension of the LBM turbulent wall model proposed by [Malaspinas and Sagaut, 2014], who developed an appropriate treatment of the unknown DF's near a wall boundary and validated the method for discretizations where the near wall node (location $\mathbf{x}_{1}$ ) is within the turbulent log law region (see Fig. 8). Here we propose a modification to their model that allows for appropriate model behavior within the log law region, linear region and transition layer, which is important for modeling curved boundaries as a large variation in sub-grid wall distance $q$ is inevitable. We then describe the modifications required for the pLBM implementation of the wall model.

We begin with a macroscopic representation of the flow within the BL and the assumptions that lead to what is commonly referred to as an "equilibrium wall model". A thin layer approximation is introduced, implying that the mean free flow is locally nearly parallel to the solid boundary (i.e., wall) and statistically stationary; it is also assumed that there is no horizontal pressure gradient. In such conditions, the mean velocity profile can be found as a function of the distance to the wall $y$ from the semi-empirical equation proposed by [Musker, 1979], on the basis of experimentally validated logarithmic "laws of the wall" for the fully turbulent upper BL, the viscous lower $\mathrm{BL}$, and a transition layer,

$$
\begin{aligned}
u_{x^{*}}\left(y^{+}\right) & =u_{\tau}\left(\left(5.424 \operatorname{atan}\left(\frac{2.0 y^{+}-8.15}{16.7}\right)\right.\right. \\
& \left.+\log _{10}\left(\frac{\left(y^{+}+10.6\right)^{9.6}}{\left(y^{+2}-8.15 y^{+}+86.0\right)^{2}}\right)-3.52\right)
\end{aligned}
$$

where the friction velocity $u_{\tau}$ and non-dimensional distance $y^{+}$are defined as,

$$
u_{\tau}=\sqrt{\tau_{w} / \rho} \quad \text { and } \quad y^{+}=y^{*} \frac{u_{\tau}}{\nu}
$$

where $x^{*}$ and $y^{*}$ define a local wall coincident coordinate system. A common model for the turbulent eddy viscosity is used

$$
\nu_{t}=[\kappa(\Delta x) q D]^{2}\left|\frac{\partial u_{x^{*}}}{\partial y^{*}}\right|
$$

where $\kappa$ is a constant chosen to be 0.384 based on experimental data and $D=\left(1-e^{\frac{-y^{+}}{26.0}}\right)$ a Van Driest damping function added to the length scale to eliminate an over prediction of the eddy viscosity near the wall [Balaras and Benocci, 1994].

\section{Turbulent Wall Model Applied to the LBM}

These macroscopic quantities calculated in the previous section are used to reconstruct the unknown DF's at fluid nodes nearest to wall nodes. Let us define $\mathbf{x}_{1}, \mathbf{x}_{2}$, and $\hat{\mathbf{n}}$ as the position 


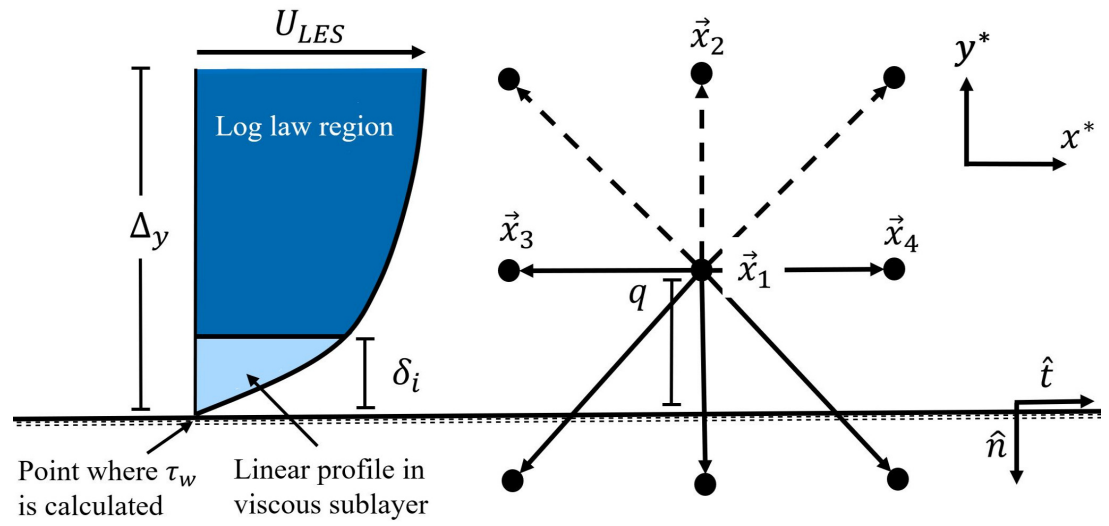

Figure 8: Sketch of LBM flow reconstruction near a solid boundary (assumed 2D for simplicity). Boundary layer profile quantities and regions are described on the left, while LBM boundary node reconstruction is seen on the right. Known (- ) and missing (- - - ) DF populations are shown, along with subgrid wall distance $q$. This image is present in a wall coincident coordinate system of $x^{*}$ and $y^{*}$ based on unit normal $(\hat{n})$ and tangential vectors $(\hat{t})$ to the wall.

of the first and second off wall lattice nodes and the outward normal unit vector at the wall, respectively (Fig. 8). As is standard in most LBM wall boundary models, DF's that satisfy $\mathbf{e}_{\alpha} \cdot \hat{\mathbf{n}}<0$ (dashed populations seen in Fig. 8) are unknown after the propagation step and must be reconstructed using $\tilde{\rho}, \tilde{\mathbf{u}}=u_{x^{*}} \hat{\mathbf{t}}$ and $\partial \tilde{\mathbf{u}} / \partial \tilde{\mathbf{y}}=-\partial \tilde{\mathbf{u}} / \partial \hat{\mathbf{n}}$.

The DFs near the wall are thus constructed as,

$$
f_{\alpha}\left(\mathbf{x}_{1}, t\right)=f_{\alpha}^{e q}(\tilde{\rho}, \tilde{\mathbf{u}})+f_{\alpha}^{n e q}\left(\frac{\partial \tilde{\mathbf{u}}}{\partial \tilde{\mathbf{y}}}\right)
$$

and $\tilde{\mathbf{y}}$ and $f_{\alpha}^{e q}$ is specified through Eq. (58) and Eq. (67) for the standard LBM or the perturbation LBM methods, respectively. In accordance with the thin boundary layer assumption, we set $\tilde{\rho}$ as equal to $\rho$ evaluated at $\mathbf{x}_{2}$. Malaspinas and Sagaut (2014) construct $f_{\alpha}^{n e q}$ as

$$
f_{\alpha}^{n e q}\left(\frac{\partial \tilde{\mathbf{u}}}{\partial \tilde{\mathbf{y}}}\right)=-\frac{w_{\alpha} \rho_{0}}{c_{s}^{2} \lambda_{\nu}} \sum_{i=1}^{3} \sum_{j=1}^{3}\left\{e_{\alpha i} e_{\alpha j}-c_{s}^{2} I_{i j}\right\} S_{i j}
$$

where $\lambda_{\nu}$ is the laminar relaxation time and $I_{i j}$ is the identity matrix. While this treatment is suitable for large $y^{+}$values, as $y^{+}$values decrease $f_{\alpha}^{n e q}$ become large relative to $f_{\alpha}^{e q}$, violating the scale assumptions within the Chapman-Enskog expansion that requires $f_{\alpha}^{e q}=\mathcal{O}(1)$ and $f_{\alpha}^{n e q}=\mathcal{O}(\epsilon)$ to recover the NS equations with the LBM. This breakdown occurs within the lower turbulent and transitional regions of the boundary layer, before the viscous sublayer is reached and a standard "bounce back" type LBM wall treatment is valid. Therefore, we apply a modification to Eq. (82) which uses a Van Driest function, $D$, to damp large values of $f_{\alpha}^{n e q}$ in a 
similar manner to Eq. (80)

$$
f_{\alpha}^{n e q}\left(\frac{\partial \tilde{\mathbf{u}}}{\partial \tilde{\mathbf{y}}}\right)=-\frac{w_{\alpha} \rho_{0} D}{c_{s}^{2} \lambda_{\nu}} \sum_{i=1}^{3} \sum_{j=1}^{3}\left\{e_{\alpha i} e_{\alpha j}-c_{s}^{2} I_{i j}\right\} S_{i j} .
$$

This modified treatment of the $f_{\alpha}^{n e q}$ produces grid convergence over a larger range of $y^{+}$values, which is essential for simulating curved boundaries on a regular lattice because large variations in $y^{*}$ are inevitable due to variational distances of the wall to lattice points. We choose to damp $f_{\alpha}^{n e q}$ at a rate of $D$, and not $D^{2}$ as in Eq. (80), based on convergence testing for the turbulent channel simulations of section 2.4.1. Finally we note that if one were to reconstruct the unknown values of $f_{\alpha}^{n e q}$ using some combination of $\sum_{\alpha}^{N} f_{\alpha}^{(1)}=\sum_{\alpha}^{N} e_{i \alpha} f_{\alpha}^{(1)}=0$ and Eq. (70) with a known velocity gradient, either an under determined or inconsistent set of equations will be found when using a D3Q19 lattice, depending on the wall orientation and number of unknowns.

\section{Model Implementation}

To evaluate the macroscopic variables of interest, Eq. (78) is solved through a NewtonRaphson scheme that iterates over $u_{\tau}$ for each near-wall lattice point, specified at location $\mathbf{x}_{\mathbf{1}}$. A tangential projection of the velocity at location $\mathbf{x}_{\mathbf{2}}$ is done such that, $U_{L E S}=\left|\mathbf{u}\left(\mathbf{x}_{\mathbf{2}}\right) \cdot \hat{\mathbf{t}}\right|(\hat{\mathbf{t}}$ being the unit local tangential vector). With $u_{\tau}$ known, $u_{x^{*}}$ and $\partial u_{x^{*}} / \partial y^{*}$ can be found with Eq. (78) and $\nu_{t}$ is found with Eq. (80) and used to calculate the relaxation rate of the 2nd-order moments as $s_{x x}=1 /\left(\tau_{0}+3 \nu_{1 T}\right)$, replacing the LES (Eq. 73).

When using this method for general boundary geometries, a shift in reference frame is needed, such that the $x$-axis points towards the local streamwise direction and locations $\mathbf{x}_{1}$ and $\mathbf{x}_{2}$ align with the wall normal. Thus, wall normal projections are applied to determine locations $\mathbf{x}_{2}$,

$$
y_{\alpha}^{*}=\frac{\mathbf{e}_{\alpha} \cdot-\hat{\mathbf{n}}}{\left|\mathbf{e}_{\alpha}\right|}
$$

The direction $\alpha$ with the largest $y_{\alpha}^{*}$ is chosen to find $\mathbf{x}_{2}=\mathbf{x}_{1}+\mathbf{e}_{\alpha} \Delta t$ and $\hat{\mathbf{n}}$ is found at the nearest point on the body to $\mathbf{x}_{1}$. When the wall is not coincident with the grid, a small loss of accuracy due to geometry is accepted as $\mathbf{e}_{\alpha}$ will not perfectly align with the wall normal.

For each $\mathbf{x}_{1}$, an evaluation of $\hat{\mathbf{n}}$ and $q$ is required at the closest point on the surface boundary to $\mathbf{x}_{1}$, which is found using a Newton-Raphson scheme that considers a boundary described as a polynomial in section 2.4 .2 and a maximum error tolerance of $\Delta x / 12$. At all $\mathbf{x}_{1}$ locations, $\hat{\mathbf{n}}$ and $q$ are computed at simulation start up. The time dependent tangential direction of the flow is found by considering the flow velocity at $\mathbf{x}_{2}\left(\mathbf{u}_{2}\right),([$ Malaspinas and Sagaut, 2014])

$$
\hat{\mathbf{t}}=\frac{\mathbf{u}_{2}-\left(\mathbf{u}_{2} \cdot \hat{\mathbf{n}}\right) \hat{\mathbf{n}}}{\left|\mathbf{u}_{2}-\left(\mathbf{u}_{2} \cdot \hat{\mathbf{n}}\right) \hat{\mathbf{n}}\right|}
$$


Finally, for curved boundaries a scenario will arise where, for a given $\mathbf{x}_{1}$, its associated $\mathbf{x}_{2}$ will also require its own separate wall model evaluation (i.e. $\mathbf{x}_{2}$ also has lattice links that cross the solid boundary) and special consideration is needed to avoid a race condition during parallel implementation.

\section{Perturbation Expansion Applied to the Wall Model Equations}

Within the wall boundary layer, the viscous component of the flow often dominates so we reconstruct the total flow equations when the perturbation LBM is applied, then remove the inviscid flow components after a total solution is found. The equilibrium DFs in Eq. (81) are now those of Eq. (67), we consider the total velocity at $\mathbf{x}_{2}$ as $U_{L E S}=\left|\left[\mathbf{u}_{2}^{I}+\mathbf{u}_{2}^{P}\right] \cdot \hat{\mathbf{t}}\right|$, and Eq. (85) now becomes

$$
\hat{\mathbf{t}}=\frac{\left(\mathbf{u}_{2}^{I}+\mathbf{u}_{2}^{P}\right)-\left(\left(\mathbf{u}_{2}^{I}+\mathbf{u}_{2}^{P}\right) \cdot \hat{\mathbf{n}}\right) \hat{\mathbf{n}}}{\left|\left(\mathbf{u}_{2}^{I}+\mathbf{u}_{2}^{P}\right)-\left(\left(\mathbf{u}_{2}^{I}+\mathbf{u}_{2}^{P}\right) \cdot \hat{\mathbf{n}}\right) \hat{\mathbf{n}}\right|}
$$

Furthermore, we now use $S_{i j}^{P}=S_{i j}-S_{i j}^{I}$ instead of $S_{i j}$ in Eq. (83), so that only the perturbation component is applied back to the DF's.

\subsection{Applications}

\subsubsection{Simulation of turbulent flow over a flat plate}

Here, we validate the turbulent wall model and LES scheme for both the LBM and pLBM by simulating a turbulent flow over a flat plate; results are compared to those of DNS simulations of [Hoyas and Jiménez, 2009] and semi-analytical solutions ([Musker, 1979]) and measurements of [Dean, 1976]. Although this benchmark has been run for the LBM and pLBM in previous work ([Malaspinas and Sagaut, 2014], [O'Reilly et al., 2016] and [O'Reilly et al., 2017]), here we present a more complete investigation that considers a wider range of discretizations, the turbulent properties of the flow, and demonstrates convergence in the calculated coefficient drag force on the wall.

We use a parallelepipedic domain of dimensions, $L=4 \pi M, H=2 M$, and $W=2 \pi M(M$ denoting the half channel width), with flat plates specified on the lower/upper boundaries at $y=0$ and $H$, on which the turbulent wall model is applied, and periodic boundary conditions in the 2 horizontal directions at $x=0$ and $L$ (streamwise) and $z=0$ and $W$ (cross stream). In this application, the flow is forced by way of a body force (term $B_{\alpha}$ in LBM Eq. 58; see, [Cabrit, 2009]), $F=\left\{u_{\tau}^{2}+u_{m}\left(u_{m}-u_{x}\right)\right\} / M$, in which $u_{x}$ is the instantaneous space-averaged downstream velocity component. The inviscid velocity field specified in the perturbation LBM is uniform over the channel, $\mathbf{u}^{I}=U$, where $U$ is calculated by applying the law of the wall, Eq. 
(78) at the center of the channel, i.e., $\tilde{u}\left(y^{+}\right)$for $y=H / 2$. The Smagorinsky constant used in the LES is $C_{S}=0.16$ in all simulations, which is in the middle of the range of recommended values. Each simulation is run until both a fully turbulent flow is observed and a quasi-steady mean flow is achieved.

We tested flows for 3 values of the Reynolds number, $\operatorname{Re}_{\tau}=M u_{\tau} / \nu=950,2,000$, and 20,000 based on the friction velocity $u_{\tau}$, or $\operatorname{Re}_{m}=2 M u_{m} / \nu=37,042,86,773$, and $1.21 \times 10^{6}$ based on the average bulk velocity $u_{m}$ in the $x$ direction, obtained from [Dean, 1976]. Each case was simulated in $4 \mathrm{LBM}$ discretizations, for which $\Delta x=\Delta y=\Delta z=M / N$, with $N=10,20$, 30 , and 40 . The full channel width is thus discretized with $2 N$ LBM points in the $y$ direction.

Fig. (9)(a-c) shows the velocity profiles computed for $\operatorname{Re}_{\tau}=950(a), 2,000(b)$ and 20,000(c) calculated with the pLBM-LES and in all cases, the pLBM-LES results agree well with [Musker, 1979]. This demonstrates the wall model's ability to accurately simulate the flow for a wide range of $y^{+}$locations of the first off-wall node. Fig. (9)(d) shows the computed bulk friction coefficient plotted verses the bulk Reynolds number, as compared to Dean's correlation [Dean, 1976],

$$
C_{f}=2 u_{\tau}^{2} / u_{m}^{2}
$$

and the upper and lower bounds of his measurements are marked to indicate the experimental variance.

In earlier turbulent channel simulations [O'Reilly et al., 2016], $C_{f}$ was calculated by using Eq. (87) where $u_{\tau}$ was found using the spatially averaged velocity within the channel and Eq. (78). Instead, a more general force evaluation approach is demonstrated here where the calculated friction coefficient is defined as $C_{f}=F_{d} /\left(\frac{1}{2} \rho u_{m}^{2} A\right)$, with $A$ being the wetted area of the top and bottom walls. The total force is calculated using a stress integration method

$$
\mathbf{F}=\iint_{A}\left\{p \hat{\mathbf{n}}+\tau_{w}\right\} d A
$$

where $\hat{\mathbf{n}}$ is the unit normal vector on the surface. Discretizing the above equation yields

$$
\mathbf{F}=\sum_{i}^{Q}\left\{p^{i} \hat{\mathbf{n}}^{i}+\tau_{w}^{i}\right\} \Delta x^{2}
$$

with, $\Delta x$ the grid spacing, and $Q$ boundary nodes. For this application the total normal component of the force will be zero. In the LBM it is common to calculate $\tau_{w}$ using the non-equilibrium component of the second-order moment (Eq. (70). This leads to a loss of accuracy from the cancellation of two close numbers in $f_{\alpha}$, and further loss of accuracy because the stress vectors acting 
(a)

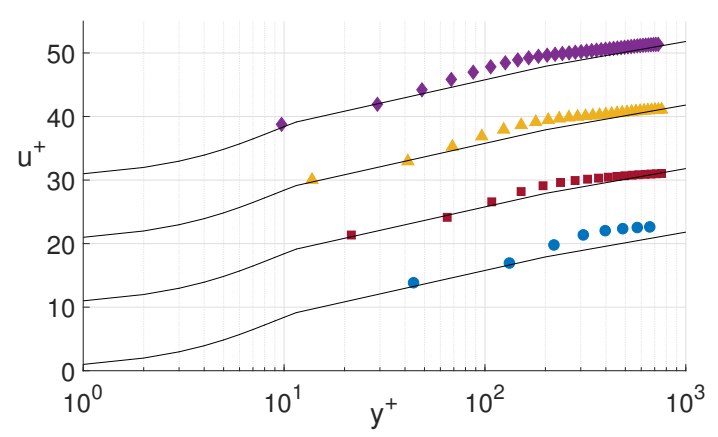

(c)

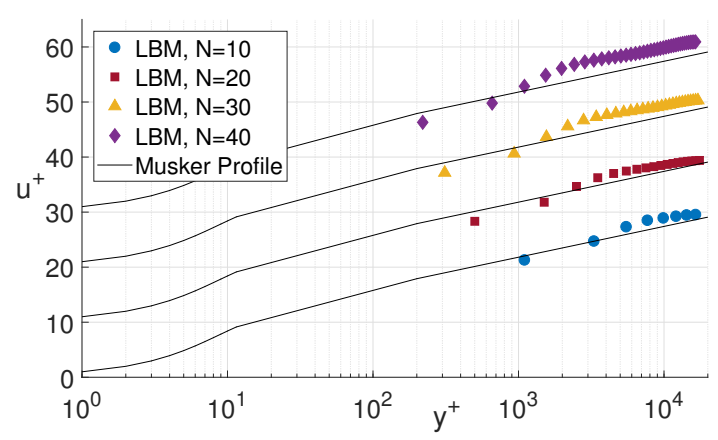

(b)

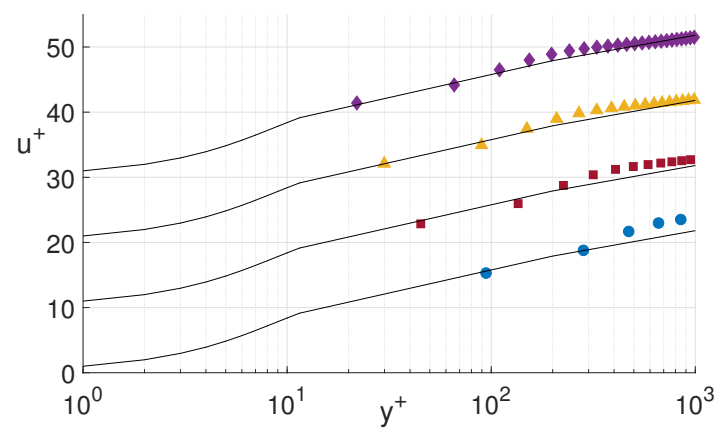

(d)

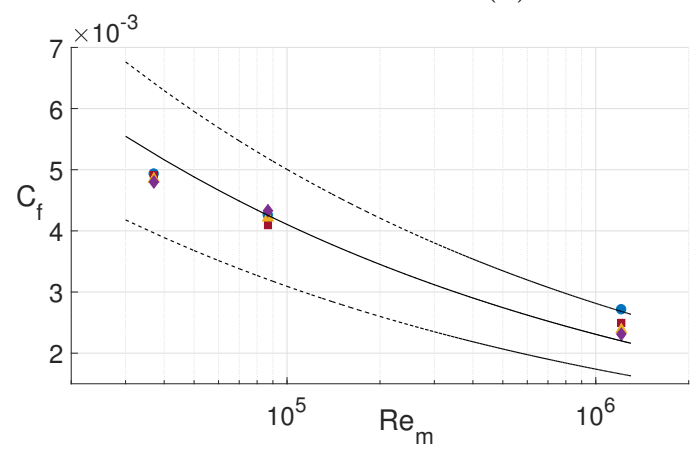

Figure 9: Mean velocity $u^{+}$as a function of distance $y^{+}$above the plate calculated using the hybrid LBM and turbulent wall model, at $\operatorname{Re}_{m}=37,042$ (a), 87,000 (b), and $1.21 \times 10^{6}$ (c). Numerical results (symbols) are plotted for a half channel of resolution $N=(\bullet) 10$, ( $\square$ ) 20, ( $\Delta$ ) 30 , and $N=40(\checkmark)$, compared to the velocity profile of [Musker, 1979] (-). For visualization purposes, results for $N=20,30$, and 40 are shifted by $\Delta u^{+}=10,20$, and 30, respectively. (d) Friction coefficient $C_{f}$ computed, compared to [Dean, 1976] (-) and the upper and lower bounds of his measurements ( - - ) as a function of Reynolds number.

on the surface of the body have to be extrapolated from the nearest lattice nodes to the boundary ([Mei et al., 2002]). Here, we eliminate this error by applying $\tau_{w}$ which has been calculated exactly at the wall boundary using the wall model.

Fig. (10) shows the turbulent velocity fluctuations and Reynolds stress in the flow for $N=40$, at $\operatorname{Re}_{m}=37,042$ (a) and 87,000 (b) with pLBM results plotted as dots and the direct NS results of [Hoyas and Jiménez, 2009] plotted as lines. While a a generally satisfactory result is observed, an under prediction of the turbulent kinetic energy in the flow is observed near to the wall which is likely the result of the equilibrium wall function chosen (Equation 78). A more advanced non-equilibum model, that considers the pressure and velocity gradients, would likely improve this result.

As stated in section 2.3, when Eq. (82) is used to calculate the non-equilibrium components in the wall model, we find divergence in the solution at the denser resolutions for $\operatorname{Re}_{\tau}=$ 
(a)

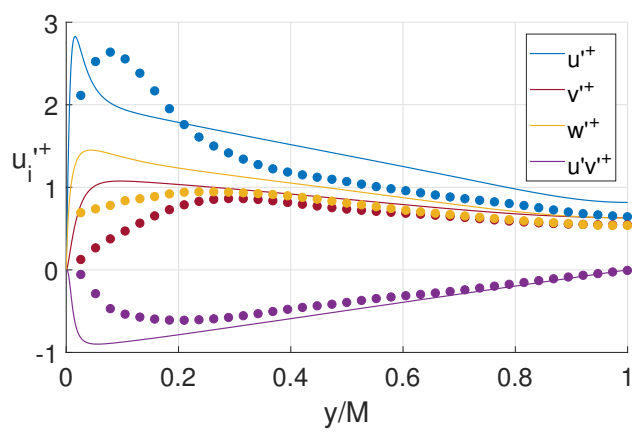

(b)

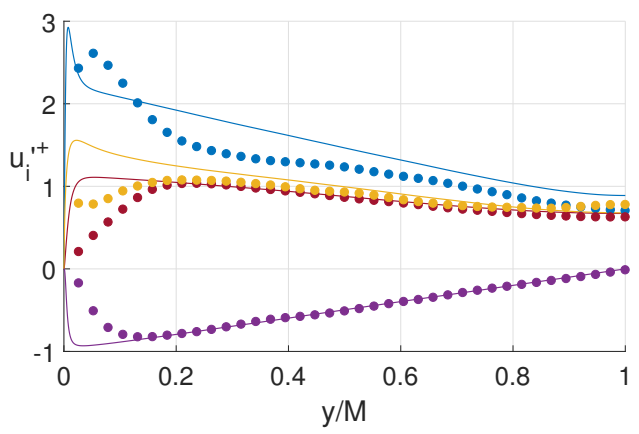

Figure 10: Turbulent fluctuation of the flow at $\operatorname{Re}_{m}=37,042$ (a) and 87,000 (b) calculated with the hybrid LBM with dots representing $u^{\prime+}(\bullet), v^{\prime+}(\bullet), w^{\prime+}(\bullet)$ and $u^{\prime} v^{\prime+}(\bullet)$ as compared to the DNS values of [Hoyas and Jiménez, 2009] (shown as lines) as a function of wall distance $y$ and calculated using a resolution $N=40$

$M u_{\tau} / \nu=950$ and 2,000, particularly in the estimation of $C_{f}$ ([Malaspinas and Sagaut, 2014], [O'Reilly et al., 2016] and [O'Reilly et al., 2017]). The results shown here demonstrate a significant improvement in wall model convergence when Eq. (83) is used. Finally, in the interest of saving space, results from the LBM-LES are not shown as almost identical results were obtained.

\subsubsection{Turbulent Foil Simulation}

Here, we focus on the simulation of the flow around a NACA0012 foil within the turbulent regime, which represents a significantly more rigorous test of the LES implementation and the turbulent wall model (TWM) due to the presence of curved boundaries and large flow gradients in both the inviscid and perturbation fields. In the following subsections, grid independence studies at $\operatorname{Re}=U C / \nu=1.44 \times 10^{6}$ (with $U$ the free flow velocity, $C$ the foil chord) are conducted at several angles of attack $(\theta)$, for $\theta=0^{\circ}, 4^{\circ}, 8^{\circ}$ using the LBM and the pLBM.

In all simulations, convergence in spatial resolution was tested using resolutions within Grid

3 of $\Delta x / C=4.0 \times 10^{-3}, 3.5 \times 10^{-3}, 3.0 \times 10^{-3}, 2.5 \times 10^{-3}$ (see Fig. 11). The turbulent wall model described in section 2.3 is applied over the entire foil surface, so it is assumed that no laminar to turbulent transition (and associated transition region) occurs as the flow moves along the foil chord. Furthermore, one may expect that a standard LBM "bounce back" type condition would be appropriate when $y^{+}$is sufficiently small (approx. $y^{+}<5$ ). However, in these simulations we found that significant pressure spikes would occur between nodes where the 2 different boundary conditions are applied. We therefore apply the TWM for all $y^{+}$values. With this setup, a maximum value of $y^{+}=292$ was found at the first off boundary nodes when simulating the coarsest 
(a)

\begin{tabular}{cccc}
\hline$\#$ & $\begin{array}{c}\text { Min. Extent } \\
(x, y, z) / C\end{array}$ & $\begin{array}{c}\text { Dimensions } \\
(x, y, z) / C\end{array}$ & $\begin{array}{c}\text { Expansion } \\
\text { Ratio }\end{array}$ \\
\hline 0 & $(-23.7-30.0-0.3)$ & $(72.0,60.0,0.8)$ & 32 \\
1 & $(-1.85-1.5-0.1)$ & $(6.0,3.0,0.4)$ & 8 \\
2 & $(-0.45-0.250 .0)$ & $(3.0,1.0,0.25)$ & 2 \\
3 & $(-0.1-0.1250 .025)$ & $(1.7,0.25,0.2)$ & 1 \\
\hline
\end{tabular}

(b)

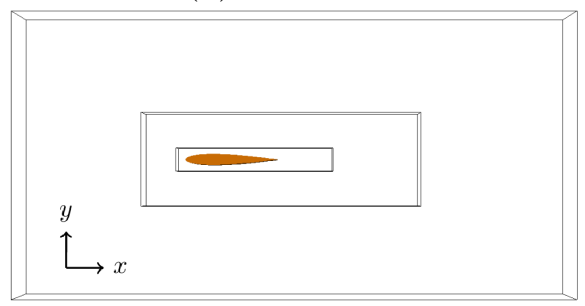

Figure 11: Nested domain set-up for the LBM and pLBM simulations of a NACA0012 foil with its leading edge defined at $x=y=z=0.0$ and chord length $C$. The foil span extends beyond the cross stream extents (z-direction) of all meshes. (a) The associated geometric parameters for each grid; the expansion ratio indicates how the mesh size $\Delta x$ changes from one grid to the other. In the pLBM simulations Grid 0 is eliminated. (b) a visualization of the of the edges (black lines) of Grids 1-3 in reference to the foil seen in orange.

mesh at $\theta=8^{\circ}$, while many boundary nodes fall within the transitional and laminar region (below $y^{+} \approx 12$ ). To ensure a smooth transition between nested grids, we enforce that at least 3 lattice nodes exist between the boundary of a domain and the boundary of a nested (finer) domain. DFs are passed between nested meshes using the methods described in [Filippova and Hänel, 2008] for the LBM and in [Janssen et al., 2018] for the pLBM. Assuming a foil chord length of $C=1$ meter and that the simulations take place in air, the free stream velocity is set to $U=21.758 \mathrm{~m} / \mathrm{s}$ and we match the physical and simulated $\mathrm{Ma}=c^{\prime} / c_{s}^{\prime}=c / c_{s}=0.050$. The associated physical time step and LBM scaling parameters can now be found by using sub-section 2.2.1.

We compare our simulation results with wind tunnel measurements and the commonly used airfoil analysis tool Xfoil [Drela and Youngren, 2001]. We use the wind tunnel measurements of Gregory and O'Reilly (1973) measured at Re $=1.44 \times 10^{6}$, for NACA0012 foils of varying roughness and Sheldahl and Klimas (2006) measured at $\mathrm{Re}=1.36 \times 10^{6}$. Free boundary layer laminar to turbulent transition is allowed for all tests and efforts were made to eliminate 3D effects such as tip vorticies during measurements. Xfoil is a 2D BEM that accounts for the boundary layer and wake using an integral boundary layer formulation. All Xfoil simulations were run to match the Mach and Reynolds numbers of our simulations using a converged 200 BEM panels on the foil surface and Xfoil simulations are set up to force a turbulent boundary layer at the leading edge of the foil.

The performance of the airfoil is evaluated with the classic pressure, lift and drag coefficients based on the pressure at the surface of the foil $(p)$, and the forces acting in the lift $\left(F_{L}\right)$ and drag 
$\left(F_{D}\right)$ directions.

$$
C_{P}=\frac{2\left(p-p_{a}\right)}{\rho_{a} U^{2} C S} \quad \text { and } \quad C_{D}=\frac{2 F_{D}}{\rho_{a} U^{2} C S} \quad \text { and } \quad C_{L}=\frac{2 F_{L}}{\rho_{a} U^{2} C S}
$$

with the ambient fluid density $\rho_{a}$, free stream velocity $U$, and with the foil span $S$ and chord $C$. In these tests forces are evaluated in the LBM and pLBM using the momentum exchange method [Janssen, 2010].

\section{LBM-LES simulation with the turbulent wall model}

To validate the LBM-LES and TWM with no inviscid forcing, boundary conditions on Grid 0 were specified as follows: (i) periodic conditions on the DFs at the sidewall boundaries $(z=-0.3 / C, 0.5 / C) ;($ ii $)$ a free stream velocity $\mathbf{u}=U$ on the inlet/top/bottom $(x=-23.7 / C$, $y= \pm 30 / C)$, prescribed by specifying the DFs as $f_{\alpha}=f_{\alpha}^{e q}\left(\rho_{a}, \mathbf{u}\right)$; (iii) zero horizontal gradient in the DFs at the outlet $(x=-23.7 / C)($ velocity $\mathbf{u}=U$ on the inlet/top/bottom $(x=-48.3 / C)$. Although not shown here, separate tests were conducted to confirm that $(x, y)$ domain boundaries were sufficiently far enough away from the foil to eliminate any nonphysical influence on the foil solution and that the domain is wide enough in the span wise direction $(z)$ to allow sufficient 3D characterization of the flow, which is particularly important for the LES of vortices.

At $\theta=0^{\circ}, 4^{\circ}$, simulations were run for 3 seconds which was sufficient to capture a steady state flow. For $\theta=8^{\circ} 5$ seconds of simulation time was required to ensure that the flow had fully developed. At the finest resolution, $\Delta x / C=2.5 \times 10^{-3}$, simulations required $\approx 5.1$ hours to compute 1 second of simulation time on a NVIDIA ${ }^{\circledR}$ Tesla ${ }^{\circledR}$ K80 GPGPU using a single precision implementation. We found that when the grid is refined beyond, $\Delta x / C=2.5 \times 10^{-3}$, double precision is required in the Newton-Rhapson iteration scheme that is used to solved Eq. (78) within the TWM. If the non-iterative TWM that has been implemented in the LBM-RANS simulations of [Wilhelm et al, 2018] is used, which uses a power law assumption of the velocity profile within the entire boundary layer, we expect an increase in computational efficiency that would be obtained at the loss of model accuracy within the linear and transitional boundary layer profile regions.

Fig. (12) shows the results of convergence testing for the LBM simulations, where the calculated forces are averaged over the last $10 \%$ of the simulation, and a converging trend towards a reasonable agreement with the measurements and Xfoil is observed in lift. Predicting lift for this foil, which is dominated by differences in pressure distribution, is in fact significantly easier than predicting drag, which is dominated by both shear and pressure forces and can be more than an 
(a)

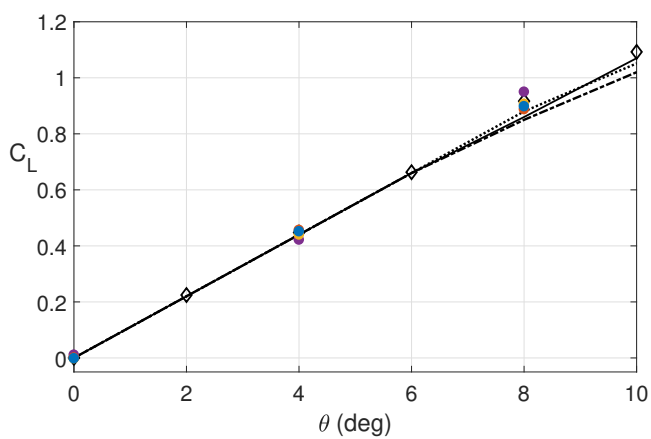

(b)

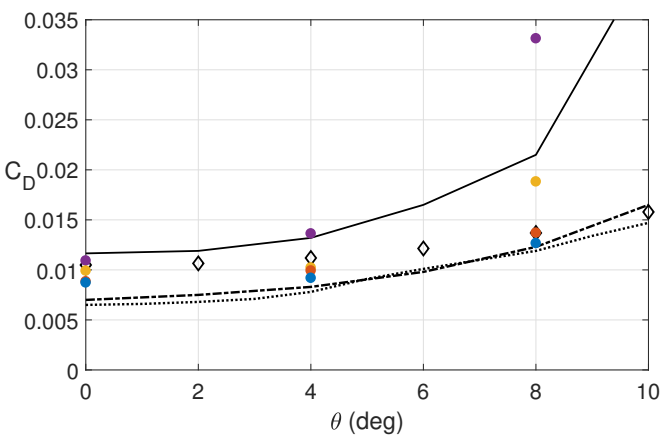

Figure 12: Lift (a) and drag (b) coefficient of a NACA0012 foil, as a function of its angle of attack. LBM simulation results, calculated at $\operatorname{Re}=1.44 \times 10^{6}$, are plotted as dots, for minimum Grid 3 resolution $(\Delta x / C)$ of: $4.0 \times 10^{-3}(\bullet), 3.5 \times 10^{-3}(\bullet), 3.0 \times 10^{-3}(\bullet), 2.5 \times 10^{-3}(\bullet)$. Xfoil simulation results are plotted as black diamonds $(\diamond)$, the measurements of [Gregory and OReilly, 1973] for $\operatorname{Re}=1.44 \times 10^{6}$ for a rough foil $(-)$, and smooth foil (- - ), and the measurements of [Sheldahl and Klimas, 2006] at $\operatorname{Re}=1.36 \times 10^{6}$ for a smooth foil $(---)$.

(a)

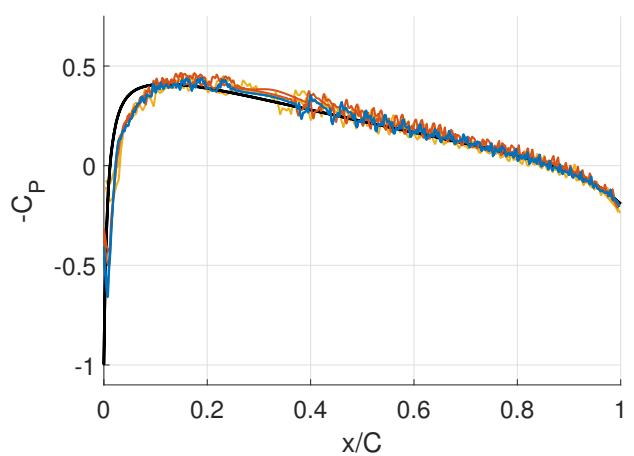

(b)

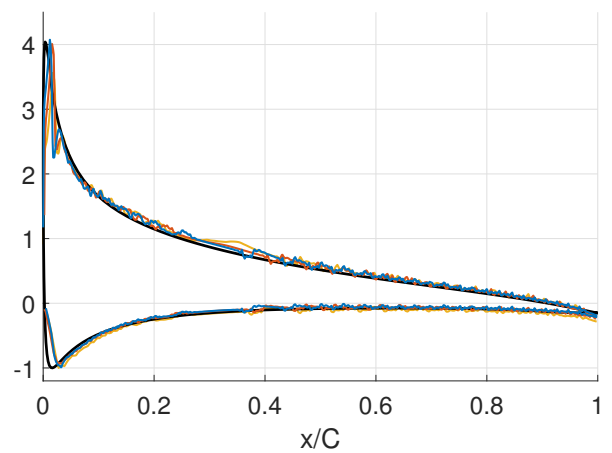

Figure 13: Negative pressure coefficients $\left(C_{p}\right)$ plotted on the surface of the NACA0012 foil, calculated at $\operatorname{Re}=1.44 \times 10^{6}$ for $\theta=0^{\circ}(\mathrm{a})$ and $\theta=8^{\circ}(\mathrm{b})$. Xfoil results (-) are compared to $\mathrm{LBM}$ results for $(\Delta x / C)=3.5 \times 10^{-3}(-), 3.0 \times 10^{-3}(-), 2.5 \times 10^{-3}(-)$.

order of magnitude smaller than lift. In the measured results of Gregory and O'Reilly (1973), the rough foil shows the highest $C_{D}$. This is because it will have both a higher overall skin fiction due to the increased roughness and the added roughness will trip a turbulent boundary layer closer to the leading edge of the foil, further increasing the viscous drag. In the LBM we assume a fully turbulent boundary layer over the entire foil but our boundary layer equations assume a perfectly smooth foil. Therefore our calculated $C_{D}$ results represent an excellent agreement with the measurements because they fall between the rough and smooth foil. This is further verified by our agreement with $X$ foil where a fully turbulent BL and smooth foil are simulated.

Fig. (13) shows convergence in the estimated $C_{P}$ calculated with the LBM and Xfoil, where 
convergence towards a good agreement with $X$ foil is observed. In the LBM results, the pressure is unknown on the surface of the body and must be extrapolated based on the pressure at the nearest LBM nodes. For these results a linear extrapolation is used. Furthermore, in most LBM simulations of the flow around a curved body, small spurious pressure oscillations will occur very near to the body. Therefore our $C_{p}$ results have an equally weighted moving average filter applied that has a window size of 7 LBM nodes. [Wilhelm et al, 2018] reduce these oscillations by modifying the body geometry so that sub-grid distances ( $q$ in Fig. 8 ) are within an advantageous region. Here we observe that a similar modification is required and limit our minimum subgrid distance that the wall model will encounter to $q_{\min }=0.1 \Delta x$. We find that decreasing this minimum allowable sub-grid distance increases these pressure errors, while increasing it allows for smoother pressure distributions at the expense of an altered geometry and, for this application, decrease in the predicted drag.

\section{pLBM-LES simulation with the turbulent wall model}

To validate the pLBM-LES and TWM along with the viscous domain reduction allowed from the hybrid method, Grid 0 is removed from the simulation, and Grid 1 is extended outward by $3 / C$ in the $\mathrm{x}$ and $\mathrm{y}$ directions. While this represents a significant domain reduction relative to the LBM, the limits of domain reduction is not tested here and the potential for further reducing the size of the pLBM domain exists. On Grid 1, boundary conditions are specified as follows: (i) periodic conditions on the DFs at the sidewall boundaries $(z=-0.1 / C, 0.3 / C)$; (ii) a zero perturbation solution that assumes $p^{P}=u_{i}^{P}=0$ at the $\mathrm{x}$ and $\mathrm{y}$ domain extents (see. [Janssen et al., 2018]). We supply a potential flow solution that is based on the KarmanTrefftz conformal mapping technique. No circulation is applied to the inviscid solution, and d'Alembert paradox says the the inviscid forces will be zero on the foil. Therefore the pLBM solution must supply a large perturbation to the inviscid flow at high angles of attack, supplying the necessary circulation to generate lift. Our Karman-Treffts foil is seen in Fig. (14)(a) and has a chord of $C_{K T}=0.9972 / C$ and thickness of $t_{K T}=0.1192 / C$ (the NACA0012 has a thickness of $12 / C)$. Our inviscid solution is found by considering the mapping function, $\zeta(z)$, that maps a complex potential flow solution for a circle, $\phi$, in the complex z-plane to a Karman-Trefftz domain ([Kerwin and Hadler 2010])

$$
\zeta(z)=\frac{\lambda a\left[(z+a)^{\lambda}+(z-a)^{\lambda}\right]}{(z+a)^{\lambda}-(z-a)^{\lambda}} .
$$


(a)

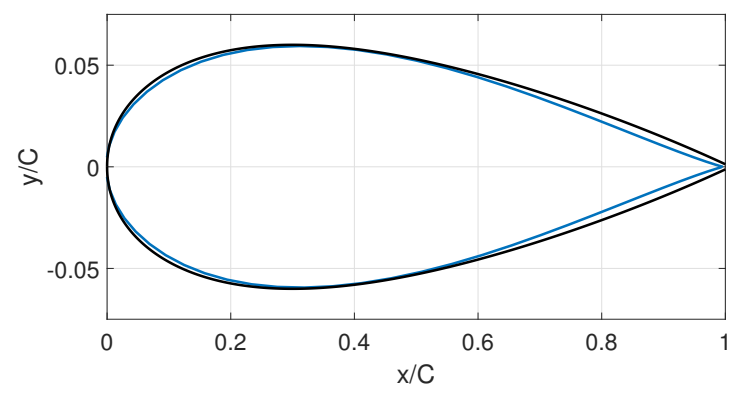

(b)

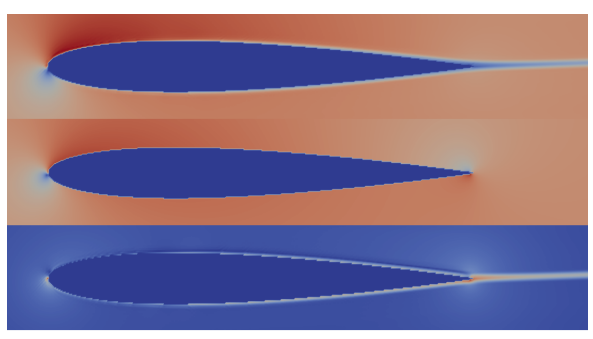

Figure 14: (a) Comparison of the Karman-Trefftz foil profile (-) used to compute the potentiaal flow solution with the NACA0012 foil profile (-). (b) Visualization of the magnitude of velocity calculated with the pLBM for a steady state and $\theta=4^{\circ}$; with total velocity, $u_{i}^{T}=u_{i}^{I}+u_{i}^{P}$ (top), the inviscid velocity, $u_{i}^{I}$, (middle), and perturbation velocity, $u_{i}^{P}$ (bottom).

with $a$, the location where the circle crosses the positive $\mathrm{x}$-axis and $\lambda$ related to the trailing edge angle $\tau$ (in degrees) through $\lambda=2-\tau / 180$. We choose the circle to be centered at $(-0.019,0.0)$ in the z-plane with a radius $r_{c}=0.273$, giving $a=r_{c}+x_{c}=0.254$. We choose a trailing edge of angle of $8.5^{\circ}$, giving $\lambda=1.9528$. The flow velocity is found from the derivative of potential solution in the $\zeta$ domain, $\partial \phi / \partial \zeta=(\partial \phi / \partial z) /(/ \partial \zeta / \partial z)$ and a visualization of it can be seen in Fig. (14)(b). More details on the Karman-Trefftz mapping can be found in [Kerwin and Hadler 2010].

Fig. (15) shows the results of convergence testing for the pLBM simulations, where the calculated forces are averaged over the last $10 \%$ of the simulation, and a converging trend towards a reasonable agreement with the LBM solution, measurements, and Xfoil is observed at the finest discretization $(\Delta x / C)=2.5 \times 10^{-3}$. This demonstrates that the pLBM is capable of capturing the lift of the foil when driven by an inviscid flow. Fig. (16) shows an example of the hybrid decomposition as applied to the pressure acting on the foil at $(\Delta x / C)=2.5 \times 10^{-3}$, where a reasonable agreement with the $X f$ foil solution is observed when considering the total pressure $p^{T}=p^{I}+p^{P}$. As was done in the LBM results of Fig. (13), a linear interpolation from the boundary nodes to the surface of the foil is applied to $p^{P}$, then an evenly weighted moving average filter of 7 nodes is applied. A visualization of the velocity decomposition $u_{i}^{T}=u_{i}^{P}+u_{i}^{I}$ can be seen in Fig. (14)(b) for $(\Delta x / C)=2.5 \times 10^{-3}$, showing that a qualitatively reasonable flow is achieved. At the finest resolution, $\Delta x / C=2.5 \times 10^{-3}$, simulations required $\approx 4.8$ hours to compute 1 second of simulation on on an NVIDIA ${ }^{\circledR}$ Tesla ${ }^{\circledR}$ K80 GPGPU, representing a slight speedup relative to the LBM results. Furthermore, the the pLBM needed to simulate less time for a steady state result to be achieved by approximately 1 second. This overall speedup is not 
(a)

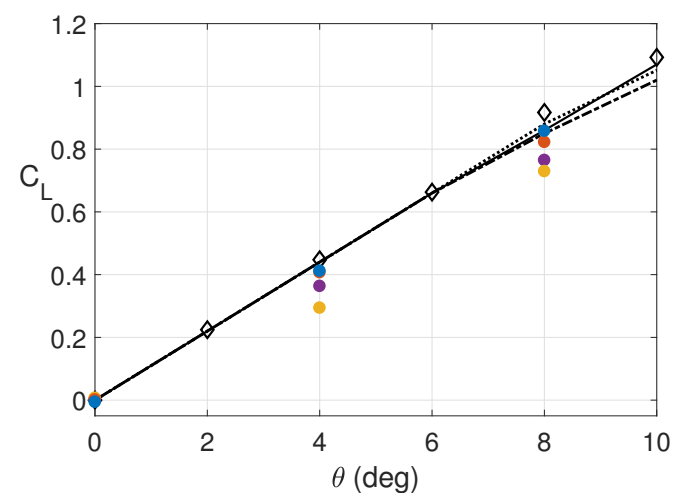

(b)

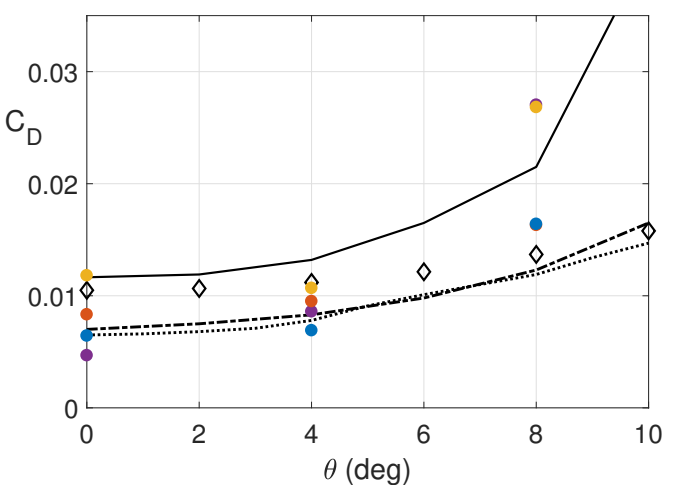

Figure 15: Lift (top) and drag (bottom) coefficient of a NACA0012 foil, as a function of its angle of attack. pLBM simulation results, calculated at $\mathrm{Re}=1.44 \times 10^{6}$, are plotted as dots, for minimum Grid 3 resolution $(\Delta x / C)$ of: $4.0 \times 10^{-3}(\bullet), 3.5 \times 10^{-3}(\bullet), 3.0 \times 10^{-3}(\bullet), 2.5 \times 10^{-3}(\bullet)$. Xfoil simulation results are plotted as black diamonds $(\diamond)$, the measurements of [Gregory and OReilly, 1973] for $\operatorname{Re}=1.44 \times 10^{6}$ for a rough foil $(-)$, and smooth foil $(--)$, and the measurements of [Sheldahl and Klimas, 2006] at $\operatorname{Re}=1.36 \times 10^{6}$ for a smooth foil $(---)$.

large because the bulk of the computational effort is in the grids near to the foil. However a greater speedup will be achieved for applications where the domain reduction can be applied in all 3 dimensions and when free surface waves must be modeled.

Limited convergence and slight under prediction in $C_{L}$ and $C_{D}$ is observed and this is a result of the differences between the KT and NACA foil geometries, where the KT foil has a smaller thickness relative to the NACA foil near the leading and trailing edges (see Fig. (14), where the geometry at these locations can significantly affect the solution. The narrow KT foil approximately between $0<x / C<0.25$ results in an under prediction of $u_{i}^{I}$ near the leading edge, which leads to an under prediction of the peak in $-C_{p}$ that is important for capturing lift, as seen in Fig. (16(d)). Near the trailing edge of the foil and at an angle of attack, high inviscid velocities are present as the inviscid flow wraps around the trailing edge (see Fig. (14)(b)). Thus a large correction must be applied by the perturbation solution, which will provide circulation to the flow. We see in Fig. (16)(b) and (d) that the perturbation component indeed provides a significant correction, but a better representation of the NACA foil geometry is required in the inviscid solution. This discrepancy at the tail of the foil is likely the cause for the lack of convergence in the highly sensitive prediction of drag and a BEM solution for the inviscid flow is required (this is being corrected for journal publication). 
(a)

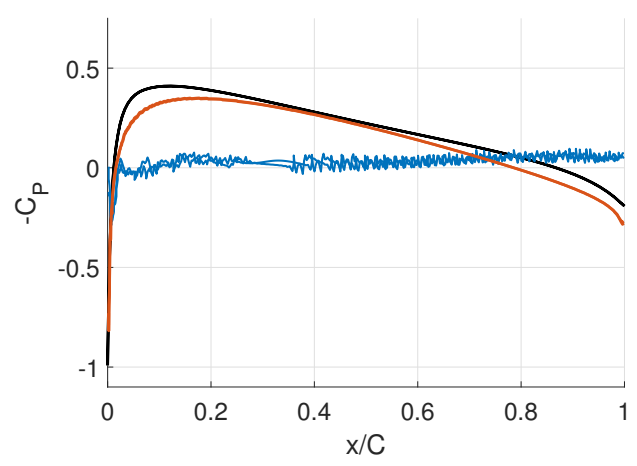

(c)

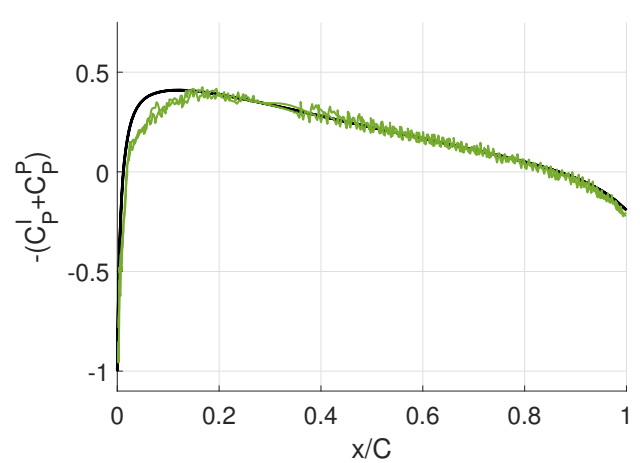

(b)

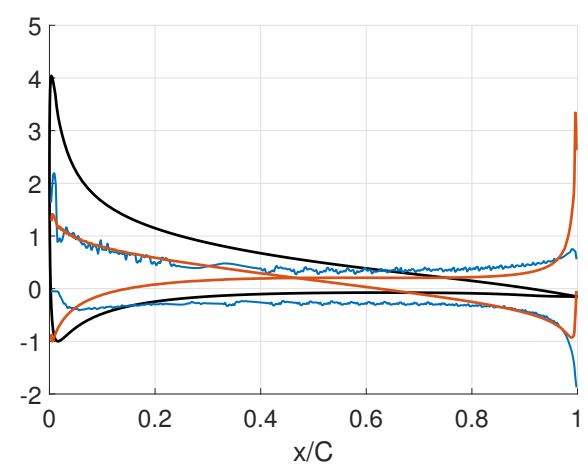

(d)

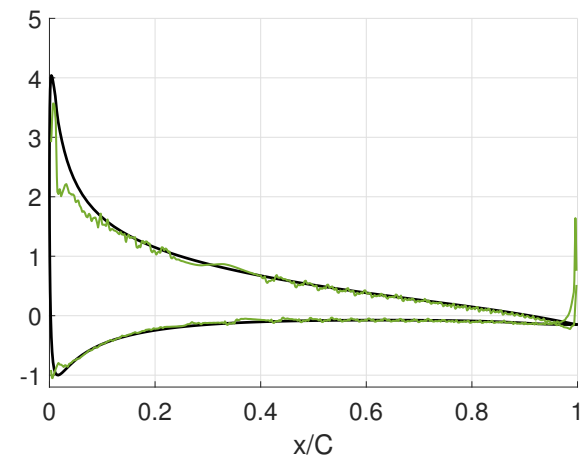

Figure 16: Negative pressure coefficients $\left(C_{p}\right)$ plotted on the surface of the NACA0012 foil, calculated at $\operatorname{Re}=1.44 \times 10^{6}$ for $\theta=0^{\circ}(\mathrm{a})(\mathrm{c})$ and $\theta=8^{\circ}(\mathrm{b})(\mathrm{d})$. Xfoil results (-) are compared to pLBM results for $(\Delta x / C)=2.5 \times 10^{-3}$ with (a) and (b) showing the perturbation component of the pressure $p^{P}$, calculated in the pLBM $(-)$ which is driven by its inviscid counterpart $p^{P}$ $(-)$ and with (c) and (d) showing the total pressure, $p^{T}=p^{I}+p^{P},(-)$.

\subsection{Conclusions}

In this paper we successfully extend our pLBM and hybrid method to simulate turbulent flows. Its potential for providing a large correction to the potential flow is demonstrated in the foil simulations by capturing lift without the need to supply circulation in the inviscid solution. This indicates the possibility for the pLBM to correct an inviscid solution when 3D effects are become large and where tip vortices and viscous spanwise flow can become important. While only a small computational speedup was achieved in the pLBM relative to the LBM in the foil simulations, a test of the limits to domain reduction was not conducted, which will be evaluated in future work with a better inviscid solution. While the potential speedup for a largely 2D simulation may not be large, we anticipate a larger hybrid speedup when the domain reduction can be applied in 3 dimensions. Further speedup is anticipated when free surface effects are introduced as the perturbation solver will no longer need to propagate waves over large distances 
away from a body.

\subsection{Acknowledgments}

C.M. OReilly, S.T. Grilli, and J.M. Dahl gratefully acknowledge support for this work from grants N000141310687 and N000141612970 of the Office of Naval Research (PM Kelly Cooper). As well as the support of XSEDE grant ENG170010 for GPU computational resources.

\subsection{Appendix A: Recovering the perturbation NS equations from the perturbation LBM}

By applying a Chapman-Enskog (CE) expansion, the macroscopic behavior of a LBM model formulation can be found from a multi scaling analysis. An expansion parameter $\epsilon$ is introduced, which is proportional to the ratio of the lattice grid $\Delta x$ to a characteristic macroscopic length (e.g., $\ell$ ). In the following, the CE scaling analysis is applied to the pEPDFs (67), by way of a perturbation expansion, which shows that these indeed allow one to recover the perturbation NS Eqs. (64).

\section{CE expansion}

Let us first consider the following quantities and scales. For convenience of notations, time derivatives $\partial / \partial t$ are denoted as $\partial_{t}$ and spatial derivatives as $\partial / \partial x_{i}$ as $\nabla_{i}$. With $\epsilon=\Delta x / \ell \ll 1$, the PDFs are expanded as follows,

$$
f_{\alpha}=f_{\alpha}^{(0)}+\epsilon f_{\alpha}^{(1)}+\epsilon^{2} f_{\alpha}^{(2)}+\mathcal{O}\left(\epsilon^{3}\right) \quad \text { with } \quad \partial_{t}=\epsilon \partial_{t_{1}}+\epsilon^{2} \partial_{t_{2}}+\mathcal{O}\left(\epsilon^{3}\right) \quad \text { and } \quad \nabla_{i}=\epsilon \nabla_{i}
$$

With these definitions, the Taylor series expansion of the first term on the LHS of Eq. (58) reads,

$$
\begin{array}{r}
f_{\alpha}\left(t+\Delta t, x_{i}+e_{\alpha i} \Delta t\right)=f_{\alpha}\left(t, x_{i}\right)+\Delta t \epsilon\left(\partial_{t_{1}}+e_{\alpha i} \nabla_{i}\right) f_{\alpha}\left(t, x_{i}\right)+ \\
\frac{\Delta t^{2}}{2} \epsilon^{2}\left(\partial_{t_{1}}+e_{\alpha i} \nabla_{i}\right)\left(\partial_{t_{1}}+e_{\alpha j} \nabla_{j}\right) f_{\alpha}\left(t, x_{i}\right)+\mathcal{O}\left(\epsilon^{3}\right),
\end{array}
$$

Defining $D_{\alpha}=\partial_{t_{1}}+e_{\alpha i} \nabla_{i}$, and collecting terms up to different orders yields to first order [Guo et al., 2002, Janssen, 2010],

$$
\mathcal{O}(1): \quad 0=-\frac{\Delta t}{\tau}\left(f_{\alpha}^{(0)}-f_{\alpha}^{e q}\right)
$$

and thus $f_{\alpha}^{0}=f_{\alpha}^{e q}$.

The PDF components of $\mathcal{O}(\epsilon)$ or smaller are then defined as the non-equilibrium components of the PDFs $\left(f_{\alpha}^{n e q}\right)$, i.e.,

$$
\mathcal{O}(\epsilon): \quad D_{\alpha} f_{\alpha}^{(0)}=-\frac{1}{\tau} f_{\alpha}^{(1)}
$$




$$
\mathcal{O}\left(\epsilon^{2}\right): \quad \partial_{t_{2}} f_{\alpha}^{(0)}+\frac{\Delta t}{2} \partial_{t 1} D_{\alpha} f_{\alpha}^{(0)}+\frac{\Delta t}{2} e_{\alpha i} \nabla_{i} D_{\alpha} f_{\alpha}^{(0)}+D_{\alpha} f_{\alpha}^{(1)}=-\frac{1}{\tau} f_{\alpha}^{(2)}
$$

Substituting Eq. (95) into Eq. (96) yields,

$$
\mathcal{O}\left(\epsilon^{2}\right): \quad \partial_{t_{2}} f_{\alpha}^{(0)}+\left(1-\frac{\Delta t}{2 \tau}\right) D_{\alpha} f_{\alpha}^{(1)}=-\frac{1}{\tau} f_{\alpha}^{(2)} .
$$

\section{PDF moments}

Computing the zeroth-order moment of Eq. (95) and considering the pEPDFs (67) recovers the conservation of mass equation for the perturbation NS equations,

$$
\begin{aligned}
\sum_{\alpha=1}^{n} \partial_{t_{0}} f_{\alpha}^{(0, P)}+\sum_{\alpha=1}^{n} e_{\alpha i} \partial_{i} f_{\alpha}^{(0, P)} & =-\frac{1}{\tau} \sum_{\alpha=1}^{n} f_{\alpha}^{(1, P)} \\
\partial_{t} \rho^{P}+\rho_{o} \nabla_{i} u_{i}^{P} & =0
\end{aligned}
$$

while the inviscid mass conservation equation is recovered when the inviscid form of the EPDFs are used,

$$
\rho_{o} \nabla_{i} u_{i}^{I}=0
$$

Taking the first-order moment of Eq. (95) and considering the pEPDFs (67) recovers the leading order terms of the perturbation NS equations,

$$
\begin{gathered}
\sum_{\alpha=1}^{n} e_{\alpha i} \partial_{t_{0}} f_{\alpha}^{(0, P)}+\sum_{\alpha=1}^{n} e_{\alpha i} e_{\alpha i} \nabla_{i} f_{\alpha}^{(0, P)}=-\frac{1}{\tau} \sum_{\alpha=1}^{n} e_{\alpha i} f_{\alpha}^{(1, P)} \\
\partial_{t} \rho_{o} u_{i}^{P}+\nabla_{i}\left(p^{P}+\rho_{o} u_{i}^{I} u_{j}^{P}+\rho_{o} u_{i}^{P} u_{j}^{I}+\rho_{o} u_{i}^{P} u_{j}^{P}\right)=0
\end{gathered}
$$

and the inviscid momentum conservation equations (Euler equations) are recovered when the inviscid form of the EPDFs of Eq. (59) are used,

$$
\partial_{t} \rho_{o} u_{i}^{I}+\nabla_{i}\left(p^{I}+\rho_{o} u_{i}^{I} u_{j}^{I}\right)=0
$$

The latter confirms that Euler equations are exactly represented in the LBM when using the inviscid form of the EPDFs in Eq. (59), $f_{\alpha}^{e q, I}$. This is unlike NS or perturbation NS equations, in which non-equilibrium components of the EPDF's must be included to represent viscous effects. Therefore, in the hybrid modeling context, this implies that an inviscid potential flow field satisfying Euler equations can be exactly mapped to the LBM variables using $f_{\alpha}^{e q, I}$. Finally, this confirms that the decomposition method used to derive Eqs. (67) does not need to consider $f_{\alpha}^{n e q, I}$ or its moments, since these are zero by definition. 
Based on these conclusions, one may infer that the numerical kinematic viscosity of the pLBM can be selected as identical to that of the standard LBM. This is confirmed by taking the first-order moment of Eq. (97), and then applying Eq. (67),

$$
\sum_{\alpha=1}^{n} e_{\alpha} \partial_{t 2} f_{\alpha}^{(0, P)}+\sum_{\alpha=1}^{n} e_{\alpha}\left(1-\frac{\Delta t}{2 \tau}\right)\left(\partial_{t_{0}}+e_{\alpha i} \nabla_{i}\right) f_{\alpha}^{(1, P)}=-\frac{1}{\tau} \sum_{\alpha=1}^{n} e_{\alpha} e_{\alpha} f_{\alpha}^{(2, P)}
$$

where the first moment of $f_{\alpha}^{(1, P)}$ is zero in the absence of a body force, and its second moment found by considering, $\epsilon \Pi^{(1, P)}=\Pi-\Pi^{(0, P)}$, with,

$$
\Pi^{(1, P)}=\sum_{\alpha=1}^{n} e_{\alpha i} e_{\alpha j} f_{\alpha}^{(1, P)}=-c_{s}^{2} \tau\left(\partial_{i} \rho_{o} u_{j}^{P}+\partial_{j} \rho_{o} u_{i}^{P}\right)
$$

and giving

$$
\partial_{t 2} \rho_{o} u_{i}^{P}-\nabla_{i}\left(\tau-\frac{\Delta t}{2}\right) c_{s}^{2}\left(\nabla_{j} \rho_{o} u_{k}^{P}+\nabla_{k} \rho_{o} u_{j}^{P}\right)=0
$$

The perturbation momentum conservation equations can now be recovered by considering Eqs. (100) and (102) to within $\mathcal{O}\left(\epsilon^{2}\right)$ and $\mathcal{O}\left(\mathrm{Ma}^{2}\right)$ as,

$$
\partial_{t} \rho_{o} u_{i}^{P}+\rho_{o} \nabla_{j}\left(u_{i}^{P} u_{j}^{P}+u_{i}^{P} u_{j}^{I}+u_{i}^{I} u_{j}^{P}\right)=-\nabla_{j} p^{P}+\nu \nabla_{j}^{2} u_{i}^{P}
$$

when the viscosity is defined as

$$
\nu=\left(\tau-\frac{\Delta t}{2}\right) c_{s}^{2}
$$

This confirms that the standard LBM relaxation time is suitable for use in the pLBM. 


\section{Bibliography}

[Alessandrini, 2007] Alessandrini, B. (2007). Thèse d'Habilitation en Vue de Diriger les Recherches. Ecole Centrale de Nantes, Nantes.

[Balaras and Benocci, 1994] Balaras E. and Benocci C. (1994). Applications of Direct and Large Eddy Simulation/ AGARD. pp. 2-1-2-6.

[Bhatnagar et al., 1954] Bhatnagar, P. L., Gross, E. P., and Krook, M. (1954). A model for collision processes in gases. i. small amplitude processes in charged and neutral one-component systems. Phys. Rev., 94(3):511-525.

[Biausser et al., 2004] Biausser B., S.T.Grilli, Fraunie P. and Marcer, R. (2004). Numerical analysis of the internal kinematics and dynamics of three-dimensional breaking waves on slopes. International Journal of Offshore and Polar Engineering, 14(4):247-256.

[Bouzidi et al., 2001] Bouzidi, M., Firdaouss, M., and Lallemand, P. (2001). Momentum transfer of a lattice-boltzmann fluid with boundaries. Physics of Fluids, 13:3452-3459.

[Brent, 1973] Brent, R. P. (1973). Algorithms for Minimization Without Derivatives. Prentice Hall. Reviewed in: American Scientist 61 (May-June 1973), 374; Mathematical Programming 4 (1973), 349; Computer J. 16 (1973), 314; Math. Comp. 28 (1974), 865-866;.

[Cabrit, 2009] Cabrit, O. (2009). Direct simulations for wall modeling of multicomponent reacting compressible turbulent flows. Physics of Fluids, 21, 055108.

[d'Humieres et al., 2002] d'Humieres, D., Ginzburg, I., Krafczyk, M., Lallemand, P., and Luo, L.-S. (2002). Multiple Relaxation-Time Lattice Boltzmann models in three-dimensions. Royal Society of London Philosophical Transactions Series A, 360:437-451.

[Dean, 1976] Dean, R. B., (1976). A single formula for the complete velocity profile in a turbulent boundary layer.. J. Fluids Eng., 98(4), 723-726.

[Drela and Youngren, 2001] Drela M. and Youngren H., (2001). Xfoil 6.94 user guide.

[Filippova and Hänel, 2008] Filippova, O., Hänel, D. (2008). Boundary-fitting and local grid refinement for lattice-BGK models. International Journal of Modern Physics, C 9 (1998) 12711279. 
[Freudiger et al., 2008] Freudiger, S., Hegewald, J., and Krafczyk, M. (2008). A parallelization concept for a multi-physics lattice boltzmann solver based on hierarchical grids. Progress in Computational Fluid Dynamics, 8(1-4):168-178.

[Frisch et al., 1987] Frisch, U., d'Humières, D., Hasslacher, B., Lallemand, P., Pomeau, Y., and Rivet, J.-P. (1987). Lattice Gas Hydrodynamics in Two and Three Dimensions. J. Complex Syst., 1:75-136.

[Geller et al., 2006] Geller, S., Krafczyk, M., Tölke, J., Turek, S., and Hron, J. (2006). Benchmark computations based on Lattice-Boltzmann, Finite Element and Finite volume Methods for laminar Flows. Comp. and Fluids, 35:888-897.

[Ginzburg and D'Humieres, 2003] Ginzburg, I. and D'Humieres, D. (2003). Multireflection boundary conditions for lattice Boltzmann models. Physical Review E, 68(6):066614.1066614.30.

[Grilli and Horrillo, 1997] Grilli, S.T. and Horrillo, J. (1997). Numerical Generation and Absorption of Fully Nonlinear Periodic Waves. Journal of Engineering Mechanics, 123(10):1060-1069.

[Grilli et al., 1997] Grilli, S.T., Svendsen, I.A. and Subramanya, R. (1997). Breaking Criterion and Characteristics for Solitary Waves on Slopes. Journal of Waterway Port Coastal and Ocean Engineering, 123(3):102-112.

[Grilli and Subramanya, 1996] Grilli, S.T. and Subramanya, R. (1996). Numerical Modeling of Wave Breaking Induced by Fixed or Moving Boundaries. Computational Mechanics, 17(6):374391.

[Grilli et al., 2001] Grilli, S., Guyenne, P., and Dias, F. (2001). A fully nonlinear model for threedimensional overturning waves over arbitrary bottom. International Journal for Numerical Methods in Fluids, 35(7):829 - 867.

[Grilli, 2008] Grilli, S.T. (2008) On the Development and Application of Hybrid Numerical Models in Nonlinear Free Surface Hydrodynamics. Keynote lecture in Proc. 8th Intl. Conf. on Hydrodynamics (Nantes, France, 9/08) (P. Ferrant and X.B. Chen, eds.), pps. 21-50.

[Grilli, 2010] Grilli, S.T., Dias, F., Guyenne, P., Fochesato, C. and F. Enet (2010). Progress in fully nonlinear potential flow modeling of 3D extreme ocean waves. Chapter 3 in Advances in 
Numerical Simulation of Nonlinear Water Waves (ISBN: 978-981-283-649-6, edited by Q.W. Ma) (Vol. 11 in Series in Advances in Coastal and Ocean Engineering). World Scientific Publishing Co. Pte. Ltd., pps. 75-128.

[Guignard et al., 1999] Guignard, S., Grilli, S.T., Marcer, R. and Rey, V. (1999). Computation of shoaling and breaking waves in nearshore areas by the coupling of BEM and VOF methods. In Proc. 9th Offshore and Polar Engng. Conf. (ISOPE99, Brest, France, May 1999), Vol. III, 304-309.

[Gueyffier et al., 1999] Gueyffier, D., Li, J., Nadim, A., Scardovelli, R., and Zaleski, S. (1999). Volume-of-fluid interface tracking with smoothed surface stress methods for three-dimensional flows. Journal of Computational Physics, 152(2):423-456.

[Guo et al., 2002] Guo, Z., Zheng, C., and Shi, B. (2002). Discrete lattice effects on the forcing term in the lattice Boltzmann method. Physical Review E, 65(4):046308.1 - 046308.6.

[Gregory and OReilly, 1973] Gregory, N., OReilly, C. L., (1973) Low-Speed Aerodynamic Characteristics of NACA 0012 Aerofoil Section, including the Effects of Upper-Surface Roughness Simulating Hoar Frost.. Ministry of De-fence Aeronautical Research Council Reports and Memoranda, No. 3726.

[Harris and Grilli, 2012] Harris, J.C. and Grilli, S.T. (2010). A perturbation approach to largeeddy simulation of wave-induced bottom boundary layer flows. Intl. J. Numer. Meth. Fluids, $68,1,574-1,604$

[Harris and Grilli, 2014] Harris, J.C. and Grilli, S.T. (2014). Large eddy simulation of sediment transport over rippled beds. Nonlin. Process. Geophys., 21, 1,169-1,184.

[Harris et al., 2016] Harris, J.C. , Dombre E., Mivehchi A., Benoit M., Grilli S.T. and Peyrard, C. (2016). Progress in fully nonlinear wave modeling for wave-structure interaction. Proc. 15th Journe de l'hydrodynamique (JH2016)., (November 22-24, Brest, France)

[He and Luo, 1997] He, X. and Luo, L.-S. (1997). Lattice boltzmann model for the incompressible navier-stokes equation. Journal of Statistical Physics, 88:927-944.

[Hirt and Nichols, 1981] Hirt, C. and Nichols, B. (1981). Volume of fluid method for dynamics of free boundaries. Journal of Computational Physics, 39:201-221. 
[Hoyas and Jiménez, 2009] Hoyas, S., Jiménez, J. (2009). Scaling of velocity fluctuations in turbulent channels up to $\operatorname{Re}_{\tau}=2000$. Phys. Fluids 18011702 .

[Janssen and Krafczyk, 2009] Janssen, C. and Krafczyk, M. (2009). A lattice Boltzmann approach for free-surface-flow simulations on non-uniform block-structured grids. Computers and Mathematics with Applications.

[Janssen, 2010] Janssen, C.F. (2010) Kinetic approaches for the simulation of non-linear free surface flow problems in civil and environmental engng. PhD thesis, Technical Univ. Braunschweig.

[Janssen et al., 2010] Janssen C.F., S.T. Grilli and M. Krafczyk (2010) Modeling of Wave Breaking and Wave-Structure Interactions by Coupling of Fully Nonlinear Potential Flow and Lattice-Boltzmann Models. Proc. 20th Offshore and Polar Engng. Conf. (ISOPE10, Beijing, China, June 20-25, 2010), pps. 686-693. Intl. Society of Offshore and Polar Engng.

[Janssen and Krafczyk, 2010] Janssen, C. and Krafczyk, M. (2010). Free surface flow simulations on GPUs using LBM. submitted to: CAMWA.

[Janssen et al., 2018] Janssen, C.F., O’Reilly C.M., Grilli S.T. (2018). A lattice boltzmann based perturbation method. submitted to: JCF.

[Junk et al., 2005] Junk, M., Klar, A., and Luo, L.-S. (2005). Asymptotic analysis of the lattice boltzmann equation. Journal of Computational Physics, 210(2):676-704.

[Körner et al., 2005] Körner, C., Thies, M., Hofmann, T., Thürey, N., and Rüde, U. (2005). Lattice boltzmann model for free surface flow for modeling foaming. Journal of Statistical Physics, 121(1-2):179-196(18).

[Kerwin and Hadler 2010] Kerwin, J.E., Hadler, J.B., (2010). The Principles of Naval Architecture Series: Propulsion. Society of Naval Architects and Marine Engineers

[Krafczyk et al., 2003] Krafczyk, M., Tölke, J., and Luo, L.-S. (2003). Large-eddy simulations with a multiple-relaxation-time LBE model. Int. J. Mod. Phys. B, 17:33-39.

[Lallemand and Luo, 2000] Lallemand, P. and Luo, L.-S. (2000). Theory of the lattice boltzmann method: Dispersion, dissipation, isotropy, galilean invariance, and stability. Physical Review, E 61:6546-6562. 
[Li et al., 2009] Li, Y., Zhang, R., Shock, R., and Chrn, H. (2009). Prediction of vortex shedding from a circular cylinder using a volumetric Lattice-Boltzmann boundary approach. The European Physical Journal Special Topics, 171, 91-97.

[Malaspinas and Sagaut, 2014] Malaspinas, O. and Sagaut, P. (2014). Wall model for large-eddy simulation based on the lattice Boltzmann method Journal of Computational Physics, 275, $25-40$

[Martin and Moyce, 1952] Martin, J. and Moyce, W. (1952). An experimental study of the collapse of liquid columns on a rigid horizontal plane. Technical report.

[Mei et al., 1999] Mei, R., Luo, L.-S., Shyy, W. (1999). An Accurate Curved Boundary Treatment in the Lattice Boltzmann Method. Journal of Computational Physics, 155(2):307-330.

[Mei et al., 2002] Mei, R., Dazhi, Y., Wei, S., Luo, L.-S. (2002). Force evaluation in the lattice Boltzmann method involving curved geometry. Physical Review E, 65(4 Pt 1):041203 May 2002

[Mei et al., 2006] Mei, R., Luo, L.-S., Lallemand, P., and d'Humieres, D. (2006). Consistent initial conditions for lattice boltzmann simulations. Computers \& Fluids, 35(8-9):855 - 862.

[Mivehchi et al., 2017] Mivehchi A., Harris, J.C., Grilli, S.T., Dahl, J.M., O'Reilly, C.M., Kuznetsov, K. and Janssen, C.F. (2017). A hybrid solver based on efficient BEM-potential and LBM-NS models: recent BEM developments and applications to naval hydrodynamics. Proc. 27th Offshore and Polar Engng. Conf.(ISOPE17, San Francsico, USA. June 2017), Intl. Society of Offshore and Polar Engng., pps. 721-728

[Musker, 1979] Musker A. (1979). Explicit expression for the smooth wall velocity distribution in a turbulent boundary layer. AIAA J., 17(6), 655-657.

[O'Reilly et al., 2015] O'Reilly, C., Grilli, S.T., Dahl, J.M., Banari, A., Janssen, C.F., Shock, J.J. and M. Uberrueck. (2015) Solution of viscous flows in a hybrid naval hydrodynamic scheme based on an efficient Lattice Boltzmann Method. Proc. 13th Intl. Conf. on Fast Sea Transportation (FAST 2015; Washington D.C., September 1-4, 2015).

[O'Reilly et al., 2016] O'Reilly C.M., Grilli S.T., Harris J.C., Mivehchi A., Janssen C.F. and J. Dahl (2016). Development of a hybrid LBM-potential flow model for Naval Hydrodynamics. Proc. 15th Journe de l'hydrodynamique (JH2016) (November 22-24, Brest, France), 15pps. 
[O’Reilly et al., 2017] O'Reilly C.M., S.T. Grilli, J.C. Harris, A. Mivehchi, C.F. Janssen and J.M. Dahl (2017). A Hybrid Solver Based on Efficient BEM-potential and LBM-NS Models: Recent LBM Developments and Applications to Naval Hydrodynamics. In Proc. 27th Offshore and Polar Engng. Conf. (ISOPE17, San Francsico, USA. June 2017), Intl. Society of Offshore and Polar Engng., pps. 713-720

[O'Reilly et al., 2018] O'Reilly C.M., S.T. Grilli, C.F. Janssen and J.M. Dahl (2018). Free surface modeling using a hybrid lattice-Boltzmann and potential flow model. To be submitted for publication

[Parker and Youngs, 1992] Parker, B. and Youngs, D. (1992). Two and three dimensional eulerian simulation of fluid flow with material interfaces. Technical report, UK Atomic Weapons Establishment.

[Quian et al., 1992] Quian, Y. H., d'Humieres, D., and Lallemand, P. (1992). Lattice BGK models for Navier Stokes equations. Europhysics Letters, 17:479-484.

[Rajani et al., 2009] Rajani, B., Kandasamy, A., and Majumdar, S. (2009). Numerical simulation of laminar flow past a circular cylinder. Journal of Applied Mathematical Modelling, 33, 12281247.

[Reliquet et al, 2014] Reliquet, G., A. Drouet, P.-E. Guillerm, L. Gentaz and P. Ferrant (2014). Simulation of wave-ship interaction in regular and irregular seas under viscous flow theory using the SWENSE method. Proc. 30th Symp. Naval Hydrod., (Tasmania, 11/2014), 11 pps.

[Salih and Moulic, 2006] Salih, A. and Moulic, S. G. (2006). A level set formulation for the numerical simulation of impact of surge fronts. SADHANA - Academy Proceedings in Engineering Sciences, 31:697-707.

[Sheldahl and Klimas, 2006] Sheldahl, R.E., and Klimas P.C. (1981). Aerodynamic characteristics of seven symmetrical airfoil sections through 180-degree angle of attack for use in aerodynamic analysis of vertical axis wind turbines. Sandia National Laboratories Energy Report, SAND80-2114

[Stålberg et al., 2006] Stålberg, E., Bruger, A., Lotstedt, P., Johansson, A., and Henningson, D. (2006). High Order Accurate Solution of Flow Past a Circular Cylinder. Journal of Scientific Computing, Vol 27. 
[Tölke, 2008] Tölke, J.(2008). Implementation of a lattice boltzmann kernel using the compute unified device architecture developed by nvidia. Computing and Visualization in Science, 1:29 $-39$.

[Tölke and Krafczyk, 2008b] Tölke, J. and Krafczyk, M. (2008b). Teraflop computing on a desktop pc with gpus for 3d cfd. International Jounrla of Computational Fluid Dynamics, 22:443 $-456$.

[Wieselsberger, 1932] Wieselsberger, C. (1932). New data on the laws of fluid resistance. Technical Notes. National Advisory Committee for Aeronautics, No. 84.

[Wilhelm et al, 2018] Wilhelm, S., Jacob, J., Sagaut, P. (2018) An explicit power-law-based wall model for lattice Boltzmann method-Reynolds-averaged numerical simulations of the flow around airfoils. Physics of Fluids, 30, 065111 (2018)

[Youngs, 1982] Youngs, D. (1982). Time-dependent multimaterial flow with large fluid distortion. In Morton, K. and Baines, M., editors, Numerical Methods for Fluid Dynamics, pages 273-285. Academic Press. 


\title{
CHAPTER 3
}

Free surface modeling using a hybrid lattice-Boltzmann and potential flow model

\author{
Christopher M. O'Reilly ${ }^{a, b}$, Stephan T. Grilli ${ }^{a}$, Christian F. Janßen ${ }^{c}$, Jason M. Dahl ${ }^{a}$ \\ ${ }^{a}$ Department of Ocean Engineering, University of Rhode Island, Narragansett, RI, USA \\ ${ }^{b}$ Navatek Ltd., South Kingstown, RI, USA \\ ${ }^{c}$ Institute for Fluid Dynamics and Ship Theory, Hamburg University of Technology, Hamburg, Germany
}

This manuscript will be submitted for publication in the future. 


\section{Abstract}

We report on the development and validation of a 3D hybrid model for naval hydrodynamics problems based on a perturbation method, in which both velocity and pressure are expressed as the sum of an inviscid flow with a viscous perturbation. The far- to near-field inviscid flows can be solved with a Boundary Element Method (BEM), based on fully nonlinear potential flow theory, and the near-field perturbation flow is solved with a NS model based on a Lattice Boltzmann Method (LBM) with a Large Eddy Simulation (LES) of the turbulence. We summarize the hybrid model formulation, present a novel hybrid volume of fluid (hVOF) approach to modeling the combined free surface of the LBM and BEM solvers, and summarize a new meshing tool for importing general geometries into the LBM. The combined LBM-BEM model is then validated by simulating the flow around a NACA0012 foil for $\mathrm{Re}=1.44 \times 10^{6}$ without a free surface and calculating the steady resistance of a Joint High Speed Sealift ship hull form using a linear free surface wave solution. Numerical errors involved in the coupling are investigated. Finally the VOF and hVOF schemes are validated by simulating a towed hydrofoil near a free surface and a nonlinear wave interacting with a cylinder with comparisons made to experiments.

\subsection{Introduction}

Numerical models simulating the irrotational motion of an incompressible, inviscid fluid, based on potential flow theory, are computationally efficient and sufficiently accurate to simulate many engineering fluid problems, such as those involving free surface waves and wave-structure interactions (e.g., [20]). However, potential flow models cannot be used in applications where viscous effects are important, for instance, in the boundary layer near solid boundaries, in the wake of bluff bodies, or to simulate surface wave breaking. Standard Computational Fluid Mechanics (CFD) Navier-Stokes (NS) solvers, such as those based on a finite volume (e.g., [31]) or Lattice Boltzmann (LBM) method (e.g., [30, 12, 40, 16, 32, 33]), can model these as well as all types of flows, but are computationally costly. Additionally, for free surface flows, NS solvers are often too numerically dissipative to model wave propagation over long distances [7].

To more efficiently solve a broad class of hydrodynamics problems of interest to many engineering disciplines, in this work, we detail the development of a high-fidelity but low cost hybrid numerical model, that combines potential flow and NS models, and applies each model in the region where it is most efficient and accurate. This hybrid model is based on a perturbation method proposed in earlier work $[2,19,37,53]$, but extends the method to free surface flow 
problems. Previously, this method was successfully used to model turbulent flows, using a finite volume method, and validated for turbulent channel and wave induced boundary layer flows [29] and demonstrated in modeling linear ship seakeeping [57]. Unlike one- or two-way coupled models applied over separate regions of a computational domain $[7,26]$, in this method both the velocity and pressure fields are expressed as the sum of inviscid/irrotational $(I)$ and viscous perturbation $(P)$ components, each solved using different numerical models in separate but overlapping computational domains. The decomposition of the flow field in this way is referred to in fluid mechanics as the Helmholtz decomposition. More specifically, the $I$ fields are solved with a potential flow model typically over a larger size domain extending to the far-field, whereas the $P$ fields are solved based on a modified (perturbed) NS equation, here with a LBM model, in a smaller near-field domain in which viscous effects are deemed important based on the considered problem (this will be made more clear later). Thus, the more computationally demanding perturbation LBM model, referred to as pLBM, is only used in the smaller near-field domain where viscous/turbulent effects matter, with its solution forced by results of the potential flow model applied to the larger domain. Hence this hybrid approach is much more computationally efficient than applying a LBM model to the entire domain, while ensuring that the complete NS solution is solved where the physics calls for it.

In engineering applications involving complex boundary conditions and/or boundary/structure geometry, the model solving potential flow equations over the entire computational domain must itself be an optimized generic numerical solver, such as based on the higher-order Boundary Element Method, and feature fully nonlinear free surface boundary conditions if applicable [34, 29].

In our work, we use a LBM to solve NS equations, instead of a finite volume solver as in earlier work, in part because the data locality and kernel simplicity of the LBM allow for a very efficient parallel implementation of the model on a "General Purpose Graphical Processor Units" (GPGPU) [35, 61, 62]. While a single GPGPU still has a limited memory, a multi-GPGPU implementation of the LBM may achieve a higher computational efficiency, for an identical accuracy, than traditional CFD solvers implemented on a massively parallel CPU cluster. In the hybrid method context, for many engineering applications, the reduced-size pLBM computational domain can often be simulated using a single GPGPU [52], allowing simulation to be run on a desktop computer equipped with a relatively inexpensive GPGPU co-processor. When the potential flow is also solved with a numerical model, e.g., BEM based, its solution may then 
be calculated using the computer's often parallelized CPUs, with limited conflicting resource requirements. If a traditional NS solver were to be used in place of the LBM, a significant number of CPU nodes would be required to run it at an accuracy equivalent to that of the LBM, leading to competing computational resources when combined with the potential flow solver.

The coupling between continuum mechanics-based equations (or models), such as potential flow, and the kinetic-based LBM is less straightforward than the earlier implementation of the hybrid method based on a volume of fluid NS solver [29]. In particular, one must derive a pLBM equivalent to the nonlinear $I-P$ coupling terms that appear in the perturbation NS equations, details are given in the following sections. To assess the ability of the LBM to simulate strongly nonlinear free surface flows, Janssen et al. $[33,34,35]$ simulated the two-dimensional (2D) "weak coupling" wave breaking results reported in earlier work [7, 26], using a LBM in combination with a Volume Of Fluid (VOF) interface tracking method. In such cases, the LBM model was simply initialized with potential flow results for waves that had been propagated up to close to the breaking point in a potential flow BEM model $[22,23,24]$. Next, the same authors computed similar results with the hybrid method, in which the $I-P$ coupling terms were represented as LBM body force terms, using the pre-computed $I$ fields to force the $P$ field solution through these terms. This approach, while proven effective, required computing spatial derivatives of both the $I$ and $P$ fields using finite difference approximations that yielded a compact but non-local LBM kernel. Additional analyses showed that this approach causes higher truncation errors in the pLBM than in the original LBM collision operator and reduces the overall efficiency of the parallelized GPGPU solution. Therefore, Janssen [33] suggested instead to introduce the nonlinear $I-P$ coupling terms directly into the LBM equilibrium probability distribution functions (EPDFs), hence, to develop perturbation EPDFs or pEPDFs. The latter were incrementally developed, implemented, and validated as part of the development of a pLBM model component to a hybrid naval hydrodynamic solver, in which the potential flow solution, with fully nonlinear free surface boundary conditions (FNPF), was computed using a higher-order BEM model $[50,51,52,37,52]$.

In this paper, we develop a hybrid free surface capturing method based on the volume of fluid (VOF) approach, where a fluid volume is tracked considering the combined flux of the $I$ and $P$ fields, yielding a total free surface in the pLBM. This leads to situations where the total free surface is either below or above the inviscid one, and the associated modifications to the free surface boundary condition, conservation equations, and pLBM variables are detailed. At 
locations where the inviscid solutions are not present, the pLBM switches to the standard LBM to simulate the full NS equations. This contrasts with other methods such as [57], where the inviscid flow solution is extrapolated at locations where the inviscid field is not present. Using the total free surface, wave breaking can be simulated in the pLBM, which can be used to eliminate wave breaking in the BEM solution. We also report on the extension of the pLBM to simulate general geometries that are not analytically defined like in previous work. This is done through the importation of a stereolithiography file and using a ray intersection routine at each pLBM node. A validation of the hybrid LBM-BEM coupling is conducted by simulating a NACA0012 foil and a Joint High Speed Sealift (JHSS) ship hull and the numerical errors associated with the coupling are discussed. The VOF and hVOF scheme is also tested by simulating a nonlinear wave interacting with a cylinder and a towed submerged hydrofoil.

\subsection{Lattice Boltzmann Method (LBM)}

In part due to its efficiency, models based on the LBM have become increasingly widely used for solving a variety of complex fluid dynamics and multi-fluid multi-physics problems (e.g., $[3,4,5])$. By contrast, with classical CFD solvers that model the macroscopic NS equations on a continuum basis, the LBM simulates CFD problems on a mesoscopic scale, in which the fluid is represented by the distribution functions (DFs) of discrete particles moving on a fixed lattice. Macroscopic hydrodynamic quantities are obtained from low-order moments of the DFs. He and Luo, [30], Lallemand and Luo [41], and d'Humieres et al. [12] discuss the LBM theory.

Besides its numerical efficiency, significant advantages of the LBM are that it exactly satisfies mass conservation and, being a pseudo-compressible method, for a single fluid there is no need to solve a pressure Poisson equation, which is typically the most time consuming part of CFD solvers. A disadvantage is the LBM low order of convergence of numerical errors (secondorder), consistent with the Chapman-Enskog expansion, which requires using smaller spatial and temporal discretization sizes than for standard CFD solvers, to achieve a similar numerical accuracy. This, however, is typically compensated for by the LBM's excellent scalability on massively parallel computer hardware [14]; GPGPU implementations of the LBM have achieved remarkable performances $[61,62,35]$. Geller et al. [16] present a study of transient laminar flows, as compared to solutions of standard CFD solvers, and Krafczyk et al. [40] discuss Large Eddy Simulations (LES), both demonstrating the efficiency and accuracy of the LBM in these contexts. 


\subsubsection{LBM fundamentals}

The primary variable of microscopic kinetic approaches is the particle distribution function (PDF) $f(t, \boldsymbol{x}, \boldsymbol{\xi})$, which specifies the normalized probability to encounter a particle at position $\boldsymbol{x}$ at time $t$, with velocity $\boldsymbol{\xi}$. The PDF evolution is described by the Boltzmann equation,

$$
\frac{D f}{D t}=\frac{\partial f(t, \boldsymbol{x}, \boldsymbol{\xi})}{\partial t}+\boldsymbol{\xi} \cdot \frac{\partial f(t, \boldsymbol{x}, \boldsymbol{\xi})}{\partial \boldsymbol{x}}=\Omega+\boldsymbol{B}
$$

whose left-hand side is an advection-type expression, the collision operator $\Omega$ describes particle interactions at the microscopic scale, and $\boldsymbol{B}$ represents body force effects.

A computationally efficient 3D model based on the Boltzmann Eq. (1) is first obtained by introducing a discretization in the velocity space $\xi$, and the resulting discrete Boltzmann equations,

$$
\frac{D f_{\alpha}}{D t}=\frac{\partial f_{\alpha}(t, \boldsymbol{x})}{\partial t}+\boldsymbol{\xi}_{\alpha} \cdot \frac{\partial f_{\alpha}(t, \boldsymbol{x})}{\partial \boldsymbol{x}}=\Omega_{\alpha}+B_{\alpha} .
$$

In this work we solve Eq. (108) on the commonly used D2Q9 and D3Q19 lattices. The former uses 9 lattice vectors, or lattice links connecting a node to its neighbors, with $\boldsymbol{e}_{\alpha}=$ $\{0,0\},\{ \pm c, 0\},\{0, \pm c\},\{ \pm c, \pm c\}, \alpha=0, \ldots, 9$ and the latter contains 19 vectors $\boldsymbol{e}_{\alpha}=$ $\{0,0,0\},\{ \pm c, 0,0\},\{0, \pm c, 0\}\{0,0, \pm c\},\{ \pm c, \pm c, 0\},\{ \pm c, 0, \pm c\},\{0, \pm c, \pm c\}, \alpha=0, \ldots, 18[55]$ with a constant velocity $c$ representing the speed of particle propagation on the lattice. Eq. (108) is now discretized in space and time using a standard first-order finite difference scheme, which yields the lattice Boltzmann equations,

$$
f_{\alpha}\left(t+\Delta t, \boldsymbol{x}+\boldsymbol{e}_{\alpha} \Delta t\right)-f_{\alpha}(t, \boldsymbol{x})=\Omega_{\alpha}+B_{\alpha}
$$

with $\Delta x$ and $\Delta t$, the spatial and temporal resolution, respectively. In the LBM, this equation is divided into a nonlinear collision step, which drives the PDFs towards a local equilibrium, and a non-local linear propagation step, where the post-collision PDFs $\left(\bar{f}_{\alpha}\right)$ are advected to neighboring nodes as,

$$
\bar{f}_{\alpha}(t, \boldsymbol{x})=f_{\alpha}(t, \boldsymbol{x})+\Omega_{\alpha}+B_{\alpha} \quad \text { and } \quad f_{\alpha}\left(t+\Delta t, \boldsymbol{x}+\boldsymbol{e}_{\alpha} \Delta t\right)=\bar{f}_{\alpha}(t, \boldsymbol{x})
$$

respectively. It has been well-established in the literature that, with the proper choice of the collision operator (see subsections 3.2.2 and 3.2.3), the solution of the lattice Boltzmann Eqs. (109) converge to that of the incompressible NS equations to within $\mathcal{O}\left(\Delta x^{2}\right)$ and $\mathcal{O}\left(\mathrm{Ma}^{2}\right)[15,38]$.

Macroscopic values of the hydrodynamic pressure $p=c_{s}^{2} \rho$ (assuming an ideal gas) and fluid 
velocity $\boldsymbol{u}$ are then found from hydrodynamic moments of the PDFs as,

$$
p(\boldsymbol{x}, t)=c_{s}^{2} \rho(\boldsymbol{x}, t)=c_{s}^{2} \sum_{\alpha=0}^{Q} f_{\alpha}(\boldsymbol{x}, t) \quad \text { and } \quad \boldsymbol{u}(\boldsymbol{x}, t)=\frac{1}{\rho} \sum_{\alpha=0}^{Q} \boldsymbol{e}_{\alpha} f_{\alpha}(\boldsymbol{x}, t)
$$

with $Q=9,18$ for our 2D and 3D lattices, respectively.

For clarity, we first consider the lattice scaling and discrete lattice effects associated with our numerical scheme. To enforce isotropy, lattice dependent weighting factors $w_{\alpha}$ (seen in Eq. (114)) are introduced as a result of using different lattice lengths $\boldsymbol{e}_{\alpha}[42]$

$$
w_{0}=\frac{1}{3}, \quad w_{1 . .6}=\frac{1}{18} \quad \text { and } \quad w_{7 . .18}=\frac{1}{36} .
$$

Both of these choices give the relationship between the speed of sound $c_{s}$ and particle velocity as $c_{s}^{2}=c / 3$.

We now consider the scaling of our physical variables to non-dimensional lattice variables (denoted by prime variables in the following) using spatial, temporal, and mass scales $\lambda, \tau$, and $\varpi$, respectively. For numerical efficiency a mesh Courant number of $C_{o}=1$ is chosen to remove the need for finite differencing in Eq. (109). In LBM, one typically assumes, $\Delta x^{\prime}=\Delta x / \lambda=1$, $\Delta t^{\prime}=\Delta t / \tau=1, c^{\prime}=c \tau / \lambda=1$, and $m^{\prime}=m / \varpi=1$.

Accordingly, our physical variables are scaled based on the flow Mach number $\mathrm{Ma}=u / c_{s}=$ $u^{\prime} / c_{s}^{\prime}$ and Reynolds number, $\operatorname{Re}=u \ell / \nu=u^{\prime} \ell^{\prime} / \nu^{\prime}$ (with $\ell$ a representative length scale of the flow) and our physical length scale, $\lambda$, becomes $\lambda=\Delta x / \ell$. Inserting $c_{s}^{\prime}=c^{\prime} / \sqrt{3}=1 / \sqrt{3}$ into the Mach number equation gives $u^{\prime}=\operatorname{Ma} \sqrt{3}$ and $\tau$ is found as $\tau=\lambda u / u^{\prime}$. Hence, the non-dimensional fluid viscosity reads, $\nu^{\prime}=\nu \tau / \lambda^{2}$. For simplicity, in the following, we will drop the prime notation for non-dimensional variables unless stated otherwise.

\subsubsection{Collision operators}

For modeling interactions between fluid particles, different collision operators $\Omega_{\alpha}$ have been proposed. In the single relaxation time (SRT) model [6], the PDFs are driven towards an equilibrium state (denoted by an $e q$ superscript) based on a single relaxation time $\tau=3 \nu / c^{2}+\Delta t / 2$, for which particle collisions are modeled as,

$$
\Omega_{\alpha}=-\frac{\Delta t}{\tau}\left\{f_{\alpha}(\boldsymbol{x}, t)-f_{\alpha}^{e q}(\rho, \boldsymbol{u})\right\}
$$

with,

$$
f_{\alpha}^{e q}(\rho, \boldsymbol{u})=w_{\alpha}\left(\rho+\rho_{o}\left(3 \frac{\left(\boldsymbol{u} \cdot \mathbf{e}_{\alpha}\right)}{c^{2}}+\frac{9}{2} \frac{\left(\boldsymbol{u} \cdot \mathbf{e}_{\alpha}\right)^{2}}{c^{4}}-\frac{3}{2} \frac{\boldsymbol{u}^{2}}{c^{2}}\right)\right)
$$


where variables $\rho_{o}$ and $\rho$ now representing the average fluid density and a small density perturbation, respectively [30].

In the more advanced and accurate MRT model [12], the PDFs and equilibrium PDFs (EPDFs) are transformed into moment space, where the PDFs are relaxed using several different relaxation rates (and times). MRT was shown to increase the stability of LBM models, particularly when applied to high Reynolds flows, which are of greater practical interest, and at the same time to enable the development of more accurate boundary conditions [17]. The moments used in the MRT $\mathbf{m}=\mathbf{M} \cdot \mathbf{f}$ are labeled as,

$$
\mathbf{m}=\left(\rho, e, \epsilon, j_{x}, q_{x}, j_{y}, q_{y}, j_{z}, q_{z}, 3 p_{x x}, 3 \pi_{x x}, p_{w w}, \pi_{w w}, p_{x y}, p_{y z}, p_{x z}, m_{x}, m_{y}, m_{z}\right)^{T}
$$

and denote the following: mass density $m_{0}=\rho$; the part of kinetic energy independent of density $m_{1}=e$; the part of kinetic energy square independent of density and kinetic energy $m_{2}=\epsilon$; momentum $m_{3,5,7}=j_{x, y, z} ; m_{4,6,8}=q_{x, y, z}$ are related to heat flux; $m_{9,11,13,14,15}$ are related to the symmetric traceless viscous stress tensor; $m_{16,17,18}$ are third-order moments; and $m_{10,12}$ are fourth-order moments. The collision operator for the MRT model is defined as,

$$
\boldsymbol{\Omega}=\mathrm{M}^{-1} \cdot \mathrm{S} \cdot\left(\mathrm{M} \cdot \mathbf{f}-\mathbf{m}^{e q}\right)
$$

where $\mathrm{M}$ denotes the transformation matrix from distribution functions to moments $(\mathbf{m}=\mathbf{M} \cdot \mathbf{f}$ and $\left.\mathbf{f}=\mathrm{M}^{-1} \cdot \mathbf{m}\right), m_{\alpha}^{e q}$ are equilibrium moments, and $\mathbf{S}=s_{\alpha, \alpha}$ is a diagonal collision matrix of relaxation parameters. The parameters,

$$
s_{9,9}=s_{11,11}=s_{13,13}=s_{14,14}=s_{15,15}=-\frac{\Delta t}{\tau}=s_{\omega}
$$

are related to the kinematic viscosity $\nu$ via the relaxation time $\tau$ as,

$$
\tau=3 \frac{\nu}{c^{2}}+\frac{1}{2} \Delta t
$$

The remaining relaxation parameters,

$$
\begin{aligned}
& s_{1,1}=s_{a}, s_{2,2}=s_{b}, s_{4,4}=s_{6,6}=s_{8,8}=s_{c}, \\
& s_{10,10}=s_{12,12}=s_{d} \quad \text { and } \quad s_{16,16}=s_{17,17}=s_{18,18}=s_{e} .
\end{aligned}
$$

can be tuned to improve the model stability [41]. While the optimal values of these parameters depend on the specific system under consideration (geometry, initial and boundary conditions), reasonable values are given in [12]. Here, we use $s_{a}=s_{b}=s_{c}=s_{d}=s_{e}=-1.0$. 


\subsubsection{Equations for the perturbation LBM}

Here, we first recap the expressions of the NS perturbation method ([19]; [29]) and develop the corresponding LBM equations with MRT. Applying a Helmholtz decomposition to the flow, both the velocity and pressure are expressed as,

$$
u_{i}=u_{i}^{I}+u_{i}^{P} \quad \text { with } \quad \tilde{p}=\tilde{p}^{I}+\tilde{p}^{P}
$$

where $\tilde{p}=p+\rho g x_{3}-\frac{2}{3} \rho k$ denotes the dynamic pressure, with $k$ the turbulent kinetic energy, modeled with a sub-grid LES model here (Eq. 120). As indicated before, superscripts $I$ denote irrotational flow quantities, with $u_{i}^{I}=\nabla_{i} \phi^{I}$ satisfying Euler equations, and superscripts $P$ represents perturbation flow quantities that are driven by the inviscid flow fields. After applying this decomposition and substituting Euler's equations, the perturbation NS equations read,

$$
\begin{aligned}
\frac{\partial u_{i}^{P}}{\partial x_{i}} & =0 \\
\frac{\partial u_{i}^{P}}{\partial t}+u_{j}^{P} \frac{\partial u_{i}^{P}}{\partial x_{j}} & =-\frac{1}{\rho} \frac{\partial \tilde{p}^{P}}{\partial x_{i}}+\left(\nu+\nu_{t}\right) \frac{\partial^{2} u_{i}^{P}}{\partial x_{j} \partial x_{j}}-\left(\frac{\partial u_{i}^{I}}{\partial x_{j}} u_{j}^{P}+u_{j}^{I} \frac{\partial u_{i}^{P}}{\partial x_{j}}\right)+2 \frac{\partial \nu_{t}}{\partial x_{j}} S_{i j}+g_{i}
\end{aligned}
$$

where $g_{i}$ represent a body force while $\nu$ and $\nu_{t}$ are kinematic molecular and turbulent viscosity, respectively, with the latter being expressed through the Smagorinsky method as,

$$
\nu_{t}=\left(C_{S} \Delta\right)^{2}|\mathbf{S}|, \quad \text { with } \quad S_{i j}=S_{i j}^{P}+S_{i j}^{I}=\frac{1}{2}\left(\frac{\partial u_{i}^{P}}{\partial x_{j}}+\frac{\partial u_{j}^{P}}{\partial x_{i}}+\frac{\partial u_{i}^{I}}{\partial x_{j}}+\frac{\partial u_{j}^{I}}{\partial x_{i}}\right)
$$

where $C_{S}$ is the Smagorinsky constant, $\Delta$ a grid filtering length scale, and $S_{i j}$ the rate of strain tensor, which here is expressed as the sum of its perturbation $S_{i j}^{P}$ and inviscid $S_{i j}^{I}$ components, both found as a function of the corresponding velocity components.

To recover Eq. (120) in the perturbation LBM, we decompose the DFs into their inviscid and perturbation components, $f_{\alpha}=f_{\alpha}^{I}+f_{\alpha}^{P}$. Introducing this decomposition into in Eq. (109) and subtracting the LBM equation for the inviscid flow, we get,

$$
\left.\left.f_{\alpha}^{P}\left(t+\Delta t, \mathbf{x}+\mathbf{e}_{\alpha} \Delta t\right)\right)-f_{\alpha}^{P}(t, \mathbf{x})\right)=-\frac{\Delta t}{\tau}\left\{f_{\alpha}^{P}(t, \mathbf{x})-f_{\alpha}^{e q}\left(\rho^{I}+\rho^{P}, \mathbf{u}^{I}+\mathbf{u}^{P}\right)+f_{\alpha}^{e q, I}\left(\rho^{I}, \mathbf{u}^{I}\right)\right\}
$$

where the $f_{\alpha}^{e q, I}\left(\rho^{I}, \mathbf{u}^{I}\right)$ can exactly reproduce Euler's equations [37]. The perturbation equilibrium DFs are then found as, $f_{\alpha}^{e q, P}\left(\rho^{P}, \mathbf{u}^{P}, \mathbf{u}^{I}\right)=f_{\alpha}^{e q}\left(\rho^{I}+\rho^{P}, \mathbf{u}^{I}+\mathbf{u}^{P}\right)-f_{\alpha}^{e q, I}\left(\rho^{I}, \mathbf{u}^{I}\right)$,

$$
f_{\alpha}^{e q, P}=w_{\alpha}\left(\rho^{P}+\rho_{o}\left(3 \frac{\mathbf{u}^{P} \cdot \mathbf{e}_{\alpha}}{c^{2}}+\frac{9}{2} \frac{\left(\mathbf{e}_{\alpha} \cdot \mathbf{u}^{P}\right)^{2}+2\left(\mathbf{e}_{\alpha} \cdot \mathbf{u}^{P}\right)\left(\mathbf{e}_{\alpha} \cdot \mathbf{u}^{I}\right)}{c^{4}}-\frac{3}{2} \frac{\left(\mathbf{u}^{P}\right)^{2}+2 \mathbf{u}^{P} \cdot \mathbf{u}^{I}}{c^{2}}\right)\right),
$$

which satisfy,

$$
\sum_{\alpha=1}^{n} f_{\alpha}^{e q, P}=\rho^{P}, \quad \sum_{\alpha=1}^{n} e_{\alpha i} f_{\alpha}^{e q, P}=\rho_{o} u_{i}^{P}, \quad \sum_{\alpha=1}^{n} e_{\alpha i} e_{\alpha j} f_{\alpha}^{e q, P}=p^{P} \delta_{i j}+\rho_{o} u_{i}^{I} u_{j}^{P}+\rho_{o} u_{i}^{P} u_{j}^{I}+\rho_{o} u_{i}^{P} u_{j}^{P} .
$$


Extending this formulation to the MRT, assuming a collision operator expressed by Eq. (115), we find the equilibrium moments,

$$
\begin{array}{r}
m_{1}^{e q, P}=e^{e q}=\rho_{0}\left(\left(u_{x}^{P}\right)^{2}+\left(u_{y}^{P}\right)^{2}+\left(u_{z}^{P}\right)^{2}+2 u_{x}^{P} u_{x}^{I}+2 u_{y}^{P} u_{y}^{I}+2 u_{z}^{P} u_{z}^{I}\right) \\
m_{9}^{e q, P}=3 p_{x x}^{e q}=\rho_{0}\left(2\left(u_{x}^{P}\right)^{2}-\left(u_{y}^{P}\right)^{2}-\left(u_{z}^{P}\right)^{2}+4 u_{x}^{P} u_{x}^{I}-2 u_{y}^{P} u_{y}^{I}-2 u_{z}^{P} u_{z}^{I}\right) \\
m_{11}^{e q, P}=p_{z z}^{e q}=\rho_{0}\left(\left(u_{y}^{P}\right)^{2}-\left(u_{z}^{P}\right)^{2}+2 u_{y}^{P} u_{y}^{I}-2 u_{z}^{P} u_{z}^{I}\right), \quad m_{13}^{e q, P}=p_{x y}^{e q}=\rho_{0}\left(u_{x}^{P} u_{y}^{P}+u_{x}^{P} u_{y}^{I}+u_{y}^{P} u_{x}^{I}\right) \\
m_{14}^{e q, P}=p_{y z}^{e q}=\rho_{0}\left(u_{y}^{P} u_{z}^{P}+u_{y}^{P} u_{z}^{I}+u_{z}^{P} u_{y}^{I}\right), \quad m_{15}^{e q, P}=p_{x z}^{e q}=\rho_{0}\left(u_{x}^{P} u_{z}^{P}+u_{x}^{P} u_{z}^{I}+u_{z}^{P} u_{x}^{I}\right)
\end{array}
$$

Moments that are not listed above are unchanged from the standard MRT formulation.

A Chapman-Enskog expansion using Eq. (122) shows that the laminar components of Eq. (120) are recovered [37] and the turbulent components can be modeled using the methodology of O'Reilly et al. (2018).

\subsection{Free Surface Boundary Conditions with the Perturbation Method}

At the free surface, the kinematic free surface boundary condition (KFSBC), represents a material derivative at the free surface

$$
\frac{\partial \eta}{\partial t}+u_{1} \frac{\partial \eta}{\partial x_{1}}+u_{2} \frac{\partial \eta}{\partial x_{2}}-u_{3}=0 \quad \text { on } x_{3}=\eta
$$

The dynamic free surface boundary condition (DFSBC) is obtained by expressing that the pressure at the free surface boundary is equal to the atmospheric pressure, $p_{a}$, and assuming zero shear stress at the free surface

$$
\tilde{p}=p+\rho g x_{3}-\frac{2}{3} \rho k=p_{a} \quad \text { on } \quad x_{3}=\eta
$$

with $\tilde{p}$ the dynamic pressure, and $k$ the turbulent kinetic energy, modeled with a sub-grid LES model (Eq. 120).

With the perturbation decomposition of Eq. (118) the total pressure may be further decomposed knowing that the inviscid solution considers gravity forcing and that the perturbation pressure contains the turbulence term.

$$
\begin{gathered}
\tilde{p}^{I}=p^{I}+\rho g z \\
\tilde{p}^{P}=p^{P}-\frac{2}{3} \rho k
\end{gathered}
$$

and by definition, $\mathbf{u}^{\prime I}=0$. Therefore nonlinear inviscid coupling is not present in this term. 
As with the Helmholtz decomposition of the velocity fields, the total free surface may be defined as an inviscid free surface plus a perturbation from that surface.

$$
\eta^{T}=\eta^{I}+\eta^{P}
$$

Within the pLBM, the total free surface is simulated so the total KFSBC must consider both the inviscid and perturbation velocities

$$
\frac{\partial \eta^{T}}{\partial t}+\left(u_{1}^{I}+u_{1}^{P}\right) \frac{\partial \eta^{T}}{\partial x_{1}}+\left(u_{2}^{I}+u_{2}^{P}\right) \frac{\partial \eta^{T}}{\partial x_{2}}-\left(u_{3}^{I}+u_{3}^{P}\right)=0
$$

This condition is satisfied through the volume of fluid method in which the total fill level is tracked as a sum of the inviscid and perturbation volume fluxes.

The hybrid DFSBC is

$$
\tilde{p}^{P}+\tilde{p}^{I}=p_{a} \quad \text { on } x_{3}=\eta^{T}
$$

Using gage pressure, we define atmospheric pressure as $p_{a}=0$. An additional constraint is that the inviscid solution enforces that $\tilde{p}^{I}=p_{a}$ at $\eta^{I}$. The hybrid DFSBC must therefore consider scenarios where the perturbation free surface is either above or below the inviscid free surface (Fig. 17) such that:

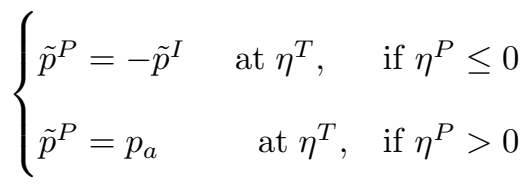

When $\eta^{P}>0$ the perturbation solution exists outside of the inviscid field and the full NS equations must be represented above the inviscid solution (Fig. 17(a)). Thus $\tilde{p}^{P}=p^{P}+\rho g_{3} x_{3}-$ $\frac{2}{3} \rho k$, and hydrostatic pressure is now present in this region. Additional modeling considerations are required at the $\eta^{I}$ interface to ensure that the conservation equations are enforced across the boundary. 
(a)

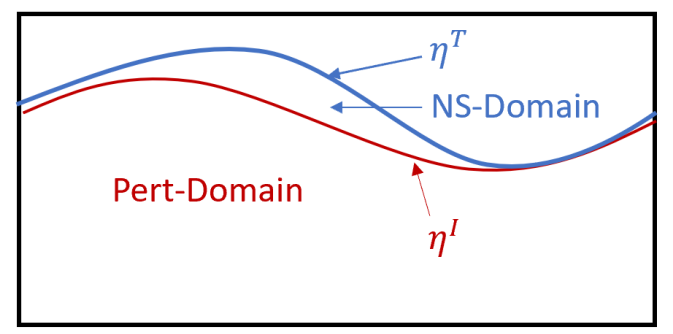

(b)

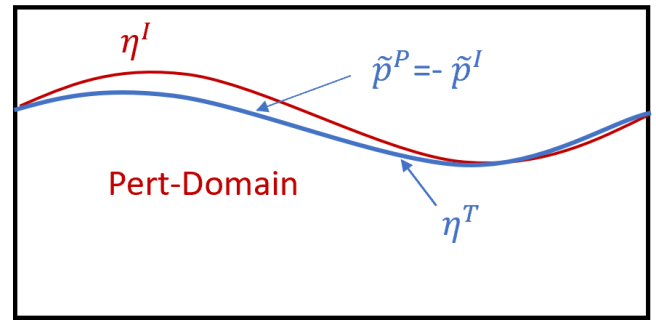

Figure 17: Visualization of the hVOF with (a) a scenario when the total free surface is above the inviscid one, $\eta^{T}>\eta^{I}$, illustrating the perturbation NS domain (red) and a region where the total NS is solved (blue). (b) a scenario when the total free surface is below the inviscid one, $\eta^{T}<\eta^{I}$ and the dynamic free surface boundary condition is modified in the pLBM.

\subsection{Hybrid Volume of Fluid Method}

The volume of fluid (VOF) method captures the interface via a fill level of a given cell,

$$
\epsilon=\frac{V_{\text {fluid }}}{V_{\text {cell }}}
$$

The VOF scheme considers cells centered at each LBM node and uses the flow quantities present at each cell center. A fill level of 0.0 marks an empty cell (gas), a fill level of 1.0 marks a full cell (fluid), while fluid and gas cells are seperated by an interface layer with a fill between 0.0 and 1.0. During time evolution, no cell may transition directly from a fluid to gas or vice versa. This is essential to conserve mass as the interface cells are where the total mass is balanced [39]. When an interface cell becomes filled $(\epsilon>1.0)$ or empty $(\epsilon<0.0)$, it becomes fluid or gas cells respectively, and new neighboring interface cells are initialized so that mass is conserved.

In the current formulation the total fill level, $\epsilon=\epsilon^{I}+\epsilon^{P}$, is considered and its discretized time evolution equation is

$$
\epsilon^{n+1}=\epsilon^{n}+\Delta \epsilon^{n}=\epsilon^{n}+\Delta \epsilon^{I, n}+\Delta \epsilon^{P, n}
$$

with $\Delta \epsilon$ representing the flux of $\epsilon$ entering or leaving the cell at time step $n$. To calculate the inviscid flux we use a finite volume formulation

$$
\Delta \epsilon^{I, n}=\Delta t \sum_{i}\left(u_{i}^{I} \hat{n}_{i}\right) A_{i}
$$

with directions $i$ that are normal to cell faces, and cell face normal $\hat{\mathbf{n}}$. The wetted area between 
the current and neighboring cells, $A_{i}$, can be estimated as an arithmetic mean of the fill level as

$$
A_{i}= \begin{cases}1.0 & : \text { fluid neighbor } \\ \frac{\epsilon(\mathbf{x}, t)+\epsilon\left(\mathbf{x}+\mathbf{e}_{\mathbf{i}}, t\right)}{2} & : \text { interface neighbor } \\ 0.0 & : \text { gas neighbor }\end{cases}
$$

The perturbation volume flux between two cells is described through an LBM formulation $[39]$

$$
\Delta \epsilon^{P, n}=\Delta t \sum_{i}\left(u_{i}^{P, n} \hat{n}_{i}\right) A_{i}^{n}=\Delta t \frac{\sum_{\alpha} \Delta m_{\alpha}^{n}}{\left(\rho_{o}+\rho^{n}\right) \Delta x^{3}}
$$

where $\rho$ represents a deviation from the average fluid density, $\rho_{o}$. A mass flux may then be calculated in terms of the DFs

$$
\Delta m_{\alpha}^{n}=\left[f_{\beta}\left(\mathbf{x}, t_{n}\right)-f_{\alpha}\left(\mathbf{x}, t_{n}\right)\right] A_{\alpha}^{n}
$$

where $\beta$ is the inverse direction to lattice direction $\alpha$ and the wetted area between the current and neighboring cells, $A_{\alpha}$, can be estimated as an arithmetic mean of the fill level as

$$
A_{\alpha}= \begin{cases}1.0 & : \text { fluid neighbor } \\ \frac{\epsilon(\mathbf{x}, t)+\epsilon\left(\mathbf{x}+\mathbf{e}_{\alpha}, t\right)}{2} & : \text { interface neighbor } \\ 0.0 & : \text { gas neighbor }\end{cases}
$$

Therefore the time evolution of the fill level for the hVOF is described by

$$
\epsilon^{n+1}=\epsilon^{n}+\Delta t \frac{\sum_{\alpha} \Delta m_{\alpha}^{n}}{\left(\rho_{o}+\rho^{n}\right) \Delta x^{3}}+\Delta t \sum_{i}\left(u_{i}^{I, n} \hat{n}_{i}\right) A_{i}^{I, n}
$$

A simple control volume analysis of a partially filled fluid cell shows that Eq. (135) reproduces the KFSBC or Hybrid KFSBC up to first order in $\Delta x$ and $\Delta t$.

\subsubsection{Dynamic Boundary Condition with the pLBM}

The pressure condition on the LBM free surface is satisfied by the anti-bounce back rule proposed by [39] at each interface node $(0.0<\epsilon<1.0)$. The procedure works by adapting the DFs so that the force exerted by the fluid is balanced by the force exerted by the surrounding air. After streaming, DFs traveling from gas to interface nodes are undefined and are constructed by using a force balance between DFs entering the fluid from the gas and vice versa. They make use of the fact that the forces exerted by the gas are known and defined by the gas pressure and velocity at the interface [39]. Unknown DFs are reconstructed as

$$
f_{\beta}^{t+1}=-f_{\alpha}^{t}+f_{\beta}^{e q}\left(\rho_{B}, \mathbf{u}_{B}\right)+f_{\alpha}^{e q}\left(\rho_{B}, \mathbf{u}_{B}\right)
$$


(a)

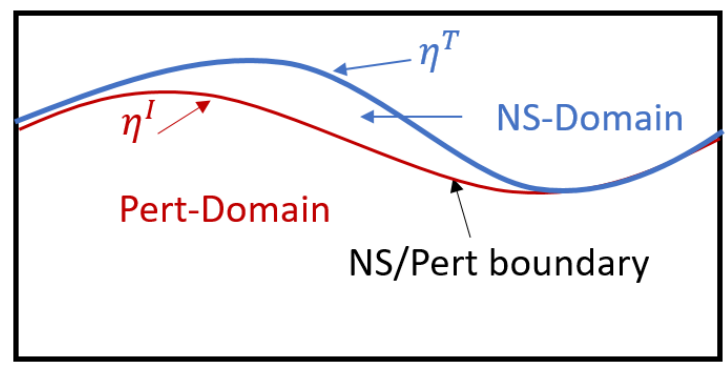

(b)

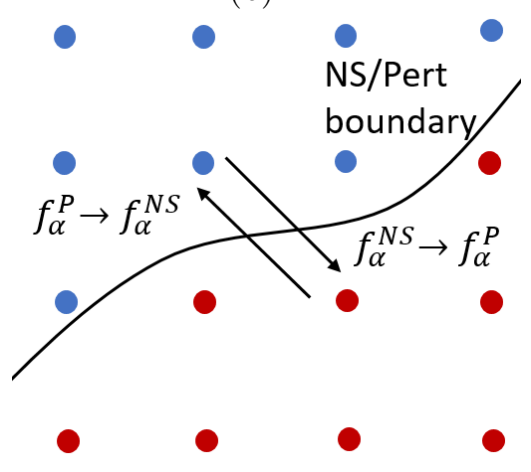

Figure 18: (a) Scenario when $\eta^{P}>0$ illustrating the interface between the perturbation NS domain and the total NS domain. (b) 2D representation of DFs crossing the perturbation NS and total NS interface.

where direction $\alpha$ corresponds to lattice directions containing gas nodes and its inverse direction $\beta$. Velocities and pressures at the free surface boundary, $\mathbf{u}_{\mathbf{B}}$ and $p_{B}$, correspond to the node's local velocity and atmospheric pressure, where $\rho_{B}=3 p_{B}$. [39] shows that when the DF are constructed in this way, the DFSBC is recovered.

For the hybrid free surface, the equilibrium DFs are replaced by their perturbation counterpart

$$
f_{\beta}^{t+1}=-f_{\alpha}^{t}+f_{\beta}^{e q}\left(\rho_{B}, \mathbf{u}_{B}^{P}, \mathbf{u}_{B}^{I}\right)+f_{\alpha}^{e q}\left(\rho_{B}, \mathbf{u}_{B}^{P}, \mathbf{u}_{B}^{I}\right)
$$

and $p_{B}$ is found from Eq. (132), while $\mathbf{u}_{B}^{P}$ is known from the pLBM solution. If $\eta^{T} \leq \eta^{I}$, the $\mathbf{u}_{B}^{I}$ is known from the inviscid solution and when $\eta^{T}>\eta^{I}, \mathbf{u}_{B}^{I}=0$ and Eq. (141) is recovered.

\subsubsection{Initialization of New Interface Nodes}

When a node transitions from gas to interface, its DFs must be initialized in a manner consistent with the conservation equations. This is done using the equilibrium DFs through

$$
\left\{\begin{array}{lll}
f_{\alpha}^{\text {new }}=f_{\alpha}^{e q}\left(\rho_{B}, \mathbf{u}_{B}^{T}\right) & \text { if } & \eta^{T}>0 \\
f_{\alpha}^{\text {new }}=f_{\alpha}^{e q}\left(\rho_{B}, \mathbf{u}_{B}^{P}, \mathbf{u}_{B}^{I}\right) & \text { if } & \eta^{T} \leq 0
\end{array}\right.
$$

where $\rho_{B}$ is specified considering the atmospheric pressure, $\mathbf{u}_{B}^{T}$ or $\mathbf{u}_{B}^{P}$ are found through a weighted average of the surrounding interface and fluid nodes, and $\mathbf{u}_{B}^{I}$ is known. When $\eta^{T}$ moves above the inviscid free surface, $\eta^{T}>\eta^{I}$, the governing equations change from the perturbation NS equations to the total NS equations. Node initialization in this scenario requires a weighted average of the surrounding interface and fluid nodes for both $\mathbf{u}_{B}^{P}$ and $\mathbf{u}_{B}^{I}$. 


\subsubsection{The Perturbation NS and Total NS interface}

When $\eta^{P}>0$, DFs will propagate through an interface separating the perturbation NS and total NS domains (Fig. 18). These DFs must be treated so that momentum is conserved for each governing equation in the next collision step. This is done by representing the inviscid solution through equlibrium DF's calculated from the inviscid flow. After each collision step, DFs are modified as

$$
\begin{cases}f_{\alpha}\left(\mathbf{x}+\mathbf{e}_{\alpha} \Delta t, t\right)=f_{\alpha}(\mathbf{x}, t)-f_{\alpha}^{e q}\left(\rho^{I}, \mathbf{u}^{I}\right) & \text { if: } f_{\alpha}(\mathbf{x}, t) \in \mathrm{NS}, f_{\alpha}\left(\mathbf{x}+\mathbf{e}_{\alpha} \Delta t, t\right) \in \mathrm{PNS} \\ f_{\alpha}\left(\mathbf{x}+\mathbf{e}_{\alpha} \Delta t, t\right)=f_{\alpha}^{P}+f_{\alpha}^{e q}\left(\rho^{I}, \mathbf{u}^{I}\right) & \text { if: } f_{\alpha}(\mathbf{x}, t) \in \mathrm{PNS}, f_{\alpha}\left(\mathbf{x}+\mathbf{e}_{\alpha} \Delta t, t\right) \in \mathrm{NS}\end{cases}
$$

where $p^{I}$ and $\mathbf{u}^{I}$ are known at the perturbation NS node.

\subsection{Power Dissipation Due to Wave Breaking}

One of the key components of the hybrid method is that wave breaking can occur in the pLBM that is driven by the BEM, which cannot model wave breaking alone. Typical nonlinear BEM models require filtering or damping at the free surface to suppress waves that would otherwise break, a process that must be carefully controlled by the user. Guignard and Grilli (2001) provide breaking suppression through an absorbing pressure patch $(\mathrm{AB})$ that provides a damping pressure term in the DFSBC to model dissipation of energy through the physical process of wave breaking. The damping pressure is defined as

$$
p_{b}(\mathbf{x}, \eta, t)=\nu_{b}(\mathbf{x}) \frac{\partial \phi}{\partial n}(\eta(\mathbf{x}, t))
$$

with, $\partial \phi / \partial n$, the normal derivative of the velocity potential at the free surface and, $\nu_{\alpha}(\mathbf{x})$, a nondimensional absorption coefficient that is based on the power dissipated during wave breaking as

$$
P_{b}(\mathbf{x}, \eta, t)=\iint_{S} p_{b} \frac{\partial \phi}{\partial n}(\eta(\mathbf{x}, t)) d A
$$

with $A$, an area on the free surface where wave breaking is occurring. Guignard and Grilli (2001) estimated $P_{b}$ based on a turbulent hydraulic jump of similar height and depth as the breaking wave.

The goal of the pLBM hVOF is to provide a more general representation $P_{b}$ and $S$ to the BEM solver where, within the hybrid domain, an AB patch is initialized in the BEM to supress wave breaking. To estimate the power dissipated in the pLBM, we use (Lamb (1932))

$$
P_{b}(\mathbf{x}, t)=\left(\mu+\mu_{t}(\mathbf{x}, t)\right) \iiint_{V}\left(\frac{\partial u_{i}(\mathbf{x}, t)}{\partial x_{j}}+\frac{\partial u_{j}(\mathbf{x}, t)}{\partial x_{i}}\right)^{2} d V
$$


with dynamic viscosity, $\mu$, an LES turbulent dynamic viscosity $\mu_{t}$, and volume $V$. Discretizing the integral into a Riemann sum and assuming grid sizes $\Delta x, \Delta y$, and $\Delta z$ gives

$$
P_{b}\left(\mathbf{x}_{m}, t_{n}\right)=4 \sum_{i}^{Q}\left(\mu+\mu_{t, m}^{n}\right)\left|\mathbf{S}_{m}^{n}\right|^{2} \Delta x_{m} \Delta y_{m} \Delta z_{m}
$$

With S given from Eq.(120), Q cells within volume V, and using time and space indices $m$ and $n$, respectively. In the pLBM the rate of strain tensor can be found by considering the second moment of the nonequilibrium PDFs ([40], [53]) within each cell $m$ and at time $n$ as

$$
\mathbf{S}=\frac{s_{x x}}{2 c_{s}^{2} \rho} \sum_{\alpha=0}^{18} \mathbf{e}_{\alpha} \mathbf{e}_{\alpha}\left(f_{\alpha}-f_{\alpha}^{e q}\right)
$$

for the D3Q19 lattice, with lattice link, $\alpha$, speed of sound $c_{s}$, relaxation time, $s_{x x}$, and equilibrium DF $f_{\alpha}^{e q}[53]$.

\subsection{Gridding General Geometries in LBM with the Turbulent Wall Model}

To utilize general body geometries with the LBM, a lattice meshing tool has been developed that identifies points within a solid boundary and, for turbulent wall model nodes, determines the boundary normal and sub-grid distance between the node and boundary. The boundary surface is represented through a stereolithography (STL) file that contains a tessellated representation of the surface that is comprised of many discrete triangles. For each triangle, the $(x, y, z)$ locations of each corner $(m)$ is given as $\mathbf{v}_{m}$ along with the surface normal $\hat{\mathbf{n}}_{\mathbf{m}}$ (pointing into the solid).

In the lattice mesher, each grid point, $\mathbf{p}$, is considered and a ray, $\mathbf{r}$, extending in a given direction is defined. The triangle intersection routine of [49] is then used for each point, ray, and triangle combination. If an intersection exists, the point is within the body when

$$
\hat{\mathbf{n}} \cdot \mathbf{r}>\mathbf{0}
$$

To calculate the sub-grid distance and normal information, an additional operation is considered for each point and triangle pair. First the centroid of the triangle is calculated as

$$
\mathbf{c}=\frac{1}{3} \sum_{n}^{3} \mathbf{v}^{t}
$$

and the distance between $\mathbf{c}$ and $\mathbf{p}$ is calculated. If this distance is less than the grid spacing, $\hat{\mathbf{n}}_{\mathbf{m}}$ and the sub-grid distance is stored at $\mathbf{p}$ and if multiple instances of this occur at the same point, the information corresponding to the minimum distance is used. We note that this calculation is not dependent on the choice of the direction of $\mathbf{r}$ and its accuracy requires sufficient accuracy in the STL geometry. 


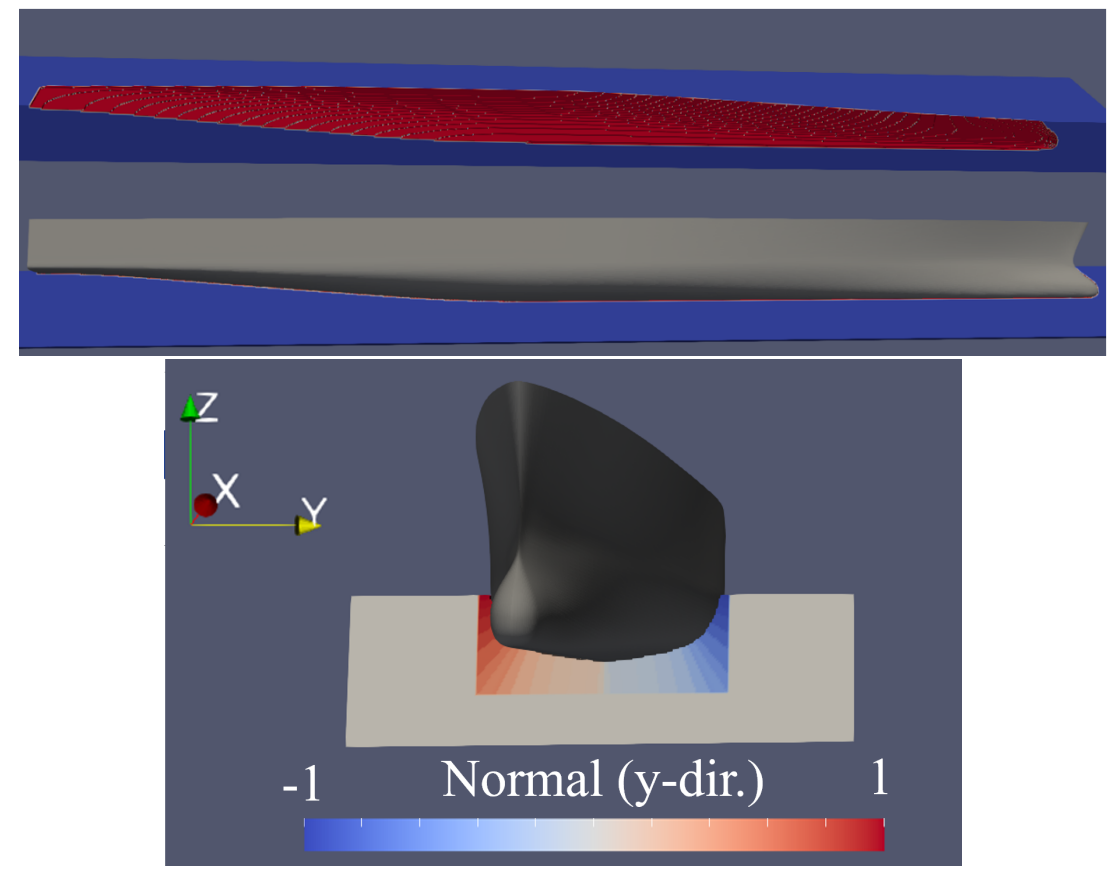

Figure 19: Snapshots of the gridded domain around a JHSS hull with (top) fluid cells shaded blue, turbulent wall model boundary cells shaded red, and the JHSS hull surface in light grey. (bottom) y-component of the normal vectors plotted for the same JHSS domain.

This "brute force" method suffers from the curse of dimensionality when meshing 3D objects as a significant increase in the number of required operations will occur as the grid and STL geometry are refined and when the number of rays increases. However, we assume a body fixed LBM mesh so this calculation is only done at simulation start up and doesn't add a major computational cost relative to the LBM simulation. Furthermore we can select a minimum number of ray directions as appropriate for a specific geometry and bounding boxes can be used to minimize the number of nodes considered.

Here we discuss the meshing process for the Joint High Speed Sealift (JHSS) ship hullform [10], seen in Fig. 19. First, a linear free surface about $z=0$ is assumed in this simulation (see below), so only points that satisfy $p_{z}<0$ are considered. Next, we observe that there is symmetry about the $\mathrm{x}-\mathrm{z}$ plane, so the calculation is done on the positive $y$ half of the domain, then mapped onto the negative side after the meshing tool is completed, switching the sign of all $y$ values on the mirrored side. Furthermore a bounding box is fit around the hull that is approximately $2 \Delta x$ larger than the maximum extents of the hull. It can be assumed that all points outside of this box are neither solid nor boundary layer nodes. With these considerations, one might expect that only one ray direction of $\mathbf{r}=(0,1,0)$ is needed to identify all points within 
the hull. However, when using dense LBM grids, rounding errors can cause small gaps between the triangles and occasionally a misclassification of a solid node is encountered. This typically occurs near the mirror plane and bilge, where adjacent triangles have a small $\left|\hat{n}_{y}\right|$. Therefore an additional search in $\mathbf{r}=(0,0,-1)$ is required, and sufficient to mesh many hull forms. However special consideration near the bulbous bow is required for the JHSS because fluid nodes above the bulb can be misclassified as solid nodes when searching in the $\mathbf{r}=(0,0,-1)$ direction. Therefore, within a small region around the bulb, Eq. (150) must be satisfied for a search in $\mathbf{r}=(0,1,0)$ and $\mathbf{r}=(0,0, \pm 1)$ directions.

\subsection{Applications}

\subsubsection{Coupling the perturbation LBM to a BEM solver}

In this section the pLBM solver is coupled to the linear ship seakeeping code Aegir, which

uses a NURBS based representation of the geometry and a high order spline representation of the hydrodynamic variables. This represents a shift from previous work ([50], [51], [52], and [53]) which used analytical inviscid solutions. Hence work shown here begins to address the additional constraints and considerations that are required for the fully coupled hybrid solver.

\section{Lifting foil simulation}

Here we simulate a NACA0012 foil operating at a Reynolds number of 1.44 million. In previous work ([50], [51], [52], and [53]), an analytical inviscid solution using conformal mapping was supplied and reasonable agreement with measurements in lift, drag and pressure distribution were demonstrated. Furthermore, no circulation was added to the inviscid solution, meaning that the perturbation component of the solution could replace the Kutta condition that is typically seen, but often faulty, in potential flow solvers.

Here the inviscid solution is calculated using Aegir, where the inviscid solution is calculated using a 3D foil of span $S=20 / C$ ( $C$ being the foil chord), 20 high order elements were used in the downstream and 40 elements were used in the cross stream directions on the top and bottom surfaces of the foil. A no penetration (Neumann) boundary condition is applied to the top and bottom surfaces of the foil while the boundary integral equations are formulated for an infinite fluid far field condition so no domain boundaries are required. No Kutta condition was added on the foil so no lift is created in the BEM solution. A 2D solution is desired and it is assumed that a nearly $2 \mathrm{D}$ result can be achieved at the center of a foil with an aspect ratio of 20 . The leading edge of the foil and half span location is centered at the origin and a sheet of points was 


\begin{tabular}{cccc}
\hline Grid & origin $(\mathrm{x} / \mathrm{C})$ & Number of nodes $(\mathrm{Nx}, \mathrm{Ny}, \mathrm{Nz})$ & $\Delta x$ \\
\hline 0 & $-0.450,-1.000,-0.075$ & $601,401,31$ & 0.005 \\
1 & $-0.350,-0.550,-0.070$ & $722,442,58$ & 0.0025 \\
\hline
\end{tabular}

Table 4: Grid parameters used for the pLBM simulation of a foil coupled to a BEM solver.

used to calculate the inviscid flow around the foil, centered at $\mathrm{z}=0$ and using only 1 node in the z-direction. Its remaining parameters match that of Grid 0 in Table (4). The pLBM solution was achieved using 2 grids defined in Table (4), the turbulent wall model of [53] supplied the body boundary conditions, a zero perturbation velocity was applied at the domain extents while periodic conditions were applied on the side. The simulation Mach number was chosen to match the physical Mach number of $\mathrm{Ma}=0.05$ in air.

An inspection of the inviscid velocity field results showed large numerical errors at points very near to the surface boundaries. This is the result of the $1 / r^{2}$ singularity that occurs in the evaluation of the integral of the Green's function on a boundary element as the distance of the point to the panel center becomes small relative to the solid angle of the panel. A common numerical cure is to successively bisect the panel, until that solid angle becomes small, then integrate over each subdivided section of the panel. This was done in Aegir, and a minimum error was achieved after 15 subdivisions were allowed on a panel, the benefit of any additional subdivisions became small relative to rounding errors. This was found to improve results, but small numerical errors near the boundaries still existed. Because the inviscid grid and Grid 0 were coincident in $\mathrm{x}$ and $\mathrm{y}$ locations no interpolation of the inviscid solution was necessary. For Grid 1 a linear interpolation was used to provide an inviscid solutions on points that were not coincident with the inviscid grid, derivatives were calculated using finite differencing, and derivatives evaluated using a point inside of the foil were removed.

The coefficient of lift $\left(C_{L}=F\left(1 / 2 \rho U^{2} C S\right)^{-1}\right)$ calculated for several angles of attack is compared to previous numerical results and measurements in Fig. (20), where a reasonable agreement in lift is observed between the pLBM coupled with the BEM and other results. Although the pLBM provided an observable correction to the flow at locations where the inviscid solution was incorrect, the inviscid error has a noticeable influence on the overall result. Furthermore, the errors observed in $C_{L}$ are of a similar magnitude to the expected drag values so drag results are not presented here. 


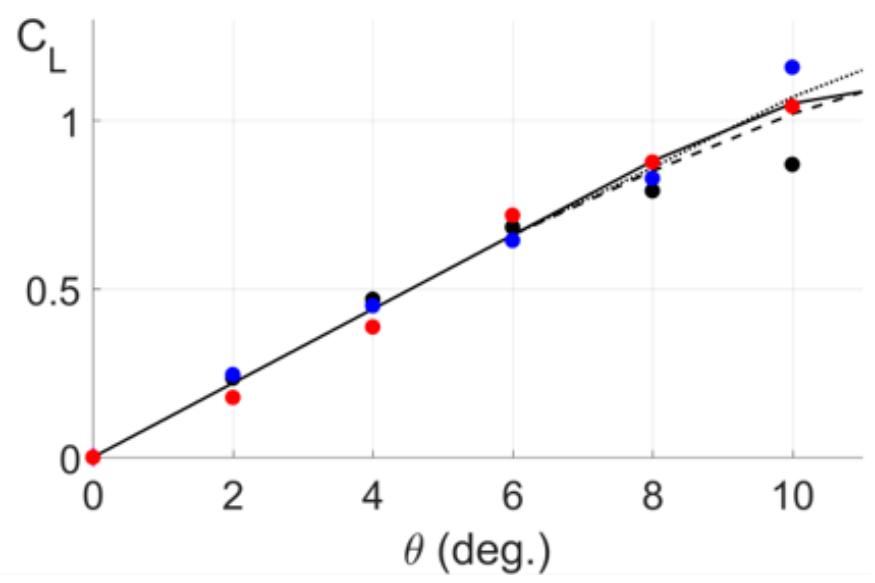

Figure 20: Coefficient of lift $\left(C_{L}=F\left(1 / 2 \rho U^{2} C S\right)^{-1}\right)$ calculated using the LBM with the turbulent wall model $(\bullet)$, the pLBM with the turbulent wall model and analytical inviscid solution $(\bullet)$, and the pLBM with the turbulent wall model and BEM inviscid solution $(\bullet)$, and compared to measurements $(-)[1]$

\section{JHSS steady resistance study}

In this test case we introduce a linear free surface, fixed at $z=0$, using the BEM solver Aegir to provide the inviscid solution. We solve for the steady resistance of a JHSS ship hullform [10] under forward motion and generating waves using the hybrid method. Simulations were run for a Froude number of $\mathrm{Fn}=U / \sqrt{g L_{W L}}=0.2,0.3,0.4$ ( $U$ is the forward speed, $g$ is gravity and $L_{W L}$ is the length of the hull waterline) with the hull fixed at the experimental sinkage and trim for each speed. The goal of the simulation is that the BEM solution will provide a very accurate estimate of the wave generating component of resistance while the pLBM will supply the viscous and form drag components. The BEM simulation was run using a mirror plane oriented along the $\mathrm{x}-\mathrm{z}$ axes and the free surface domain covered $\left(x_{\min } / L_{L W L}, x_{\max } / L_{L W L}\right)=(-1.1,0.76)$ and $\left(y_{\min } / L_{L W L}, y_{\max } / L_{L W L}\right)=(0,0.49)$ with a regular free surface panel size of $0.014 / L_{W L}$. The wetted surface of the hull was discretized with 76 panels in the downstream direction and 6 panels in the cross stream direction and the center of mass of the hull was located at the $\mathrm{x}-\mathrm{y}$ origin. A grid independent solution was confirmed for these simulation parameters and a visualization of the Aegir result at $F n=0.3$ can be seen in Fig (22).

The pLBM simulation used a reduced domain consisting of the 2 grids described in Fig (21) that were run on 2 separate GPUs, in parallel. Grid 1 represents approximately the maximum number of nodes that can be used on the GPU, containing 12 GB of memory. Because a linear free surface is assumed in the Aegir solution, the free surface is set at $z=0$ and the VOF solver 


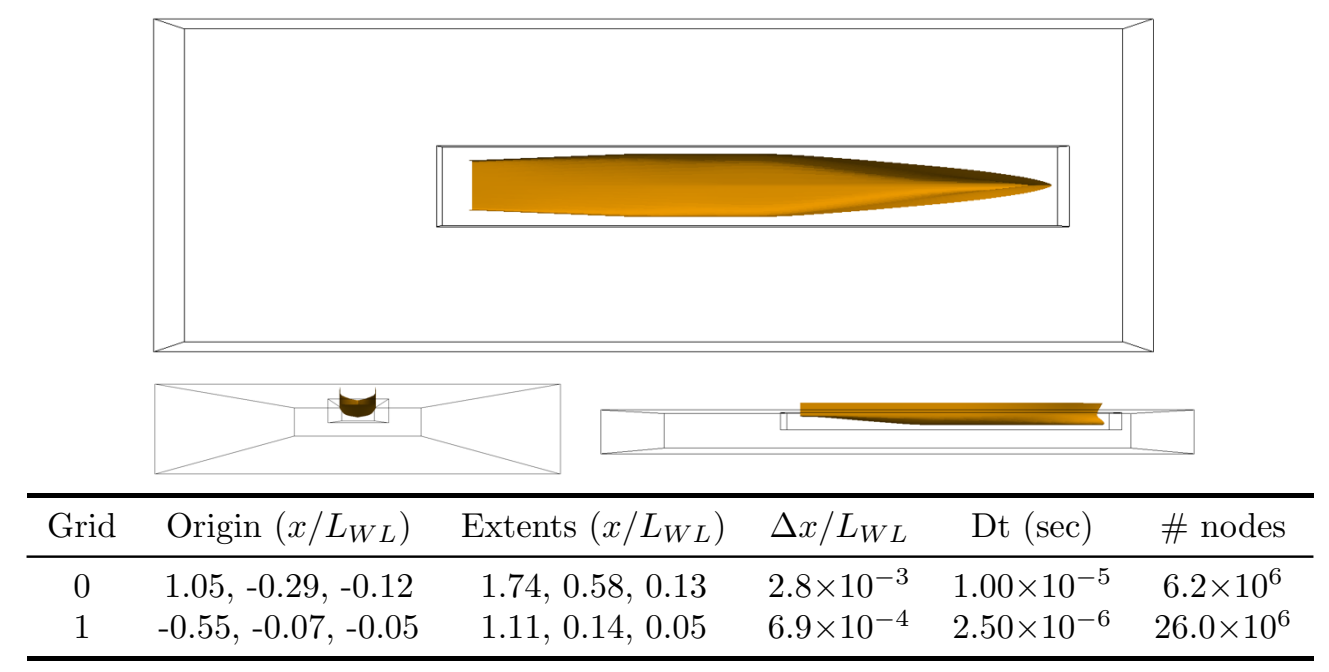

Figure 21: (top) A visualization of the pLBM domain used in the JHSS steady resistance simulation, black lines in the image represent the outer extents of each grid and the hull is seen in orange. (bottom) pLBM domain parameters for the JHSS steady resistance simulation.

is not used. Instead, an atmospheric pressure boundary condition is applied to the free surface boundary. The hybrid turbulent wall model was used as a hull boundary condition, with an estimated average $y^{+}=300$ with the hybrid LES turbulence model applied [53]. When using the LES turbulence model, a mirror boundary at $y=0$ is not valid, and the full domain must be simulated. A zero perturbation, $\rho^{P}=u_{i}^{I}=0$ boundary condition was applied to the remaining edges of the domain. The inviscid velocity field was gridded to match Grid 0, and as done in the previous test, a linear interpolation was used to provide an inviscid solutions on points that were not coincident with the inviscid grid in Grid 1. We note that derivatives were calculated using finite differencing and derivatives evaluated using a point inside of the hull were removed. As observed in the previous application, inviscid velocity errors were present at points that were very close to the free surface or hull.

Simulation results and snapshots can be seen in Fig. (24). Due to the relatively high Reynolds numbers, and orientation of the forward motion, no separation of the flow was expected. The significant perturbation components of the flow are therefore localized to a very small region around the hull. The perturbation solution also has a very small solution near the free surface meaning that it will not contribute significantly to the wave drag component of the solution, with an exception to a localized region behind the transom. We expect that nearly all of the pressure drag should be captured in the inviscid result, with very small viscous perturbation pressure, or form drag. However, pressure spikes in the perturbation solution were created from 
(a)

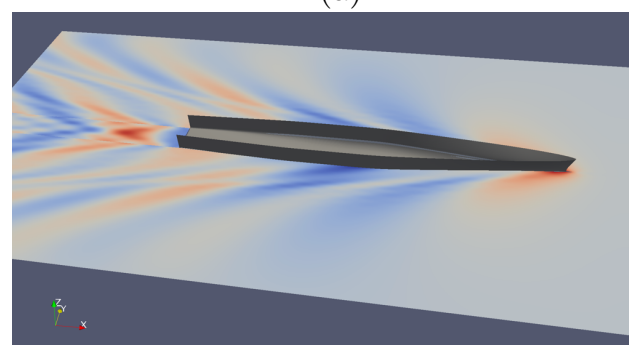

(b)

\begin{tabular}{cccc}
\hline & Run 1 & Run 2 & Run 3 \\
\hline Fn & 0.2 & 0.3 & 0.4 \\
Re & $15.5 \times 10^{6}$ & $23.4 \times 10^{6}$ & $31.0 \times 10^{6}$ \\
Ma & 0.0012 & 0.002 & 0.0024 \\
\hline
\end{tabular}

Figure 22: (a) Visualization of the free surface elevation predicted by the BEM solver Aegir using a linear free surface at $\mathrm{Fn}=0.3$. The JHSS hull can be seen in grey and for visualization purposes the sufaces are mirrored about the $\mathrm{y}=0$ plane. (b) Relevant non-dimensional physical parameters of each hybrid method run for the JHSS steady resistance simulations.

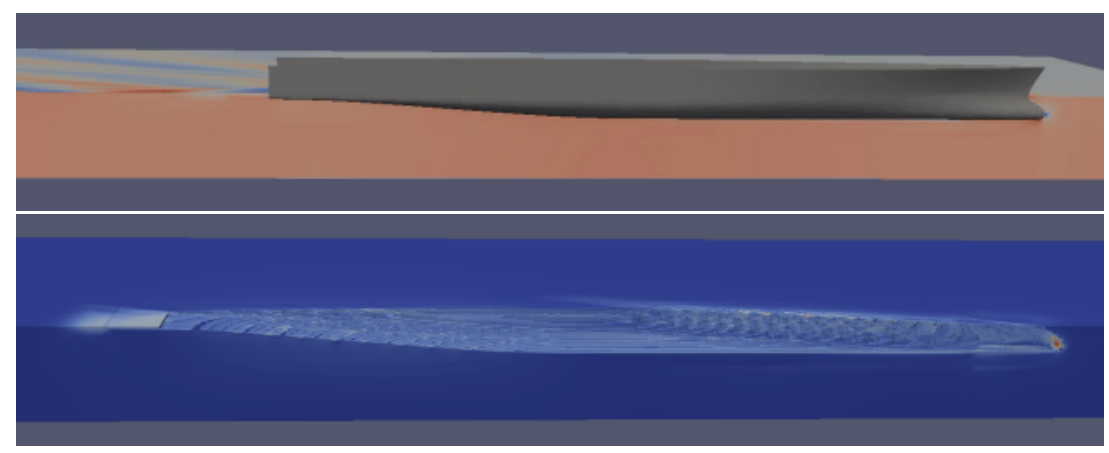

Figure 23: Snapshots of the JHSS steady resistance simulation using the hybrid method running at a $\mathrm{Fn}=0.3$. with the hybrid domain cut along $y=0$ and $z=0$. (top) Visualization of the total hybrid method solution, with the linear free surface elevation (BEM) shown at $z=0$ and total velocity magnitude $u_{i}=u_{i}^{I}+u_{i}^{P}(\mathrm{BEM}+\mathrm{pLBM})$ shown along the $y=0$ plane, and the JHSS hull seen in grey. (bottom) Visualization of the perturbation velocity magnitude $u_{i}^{P}$ (pLBM) the domain is cut along the $y=0$ plane and only water and turbulent wall model nodes are shown.

the errors of the inviscid solution (seen at the bulbous bow in Fig. 22), causing an over prediction of the perturbation pressure drag. Overall, the pLBM reasonably predicts the viscous drag, and a comparison of the drag prediction as compared to tow tank measurements and its different components of the calculated drag can be seen in Fig. (24). An improved inviscid velocity field is required for a more accurate viscous pressure estimate.

\subsubsection{Nonlinear free surface modeling}

In this section the VOF scheme is validated and tested using the LBM and pLBM. The VOF scheme used based on what is described in [33], which has been validated for simulations of a dam break and an overturning wave on a sloping beach. Here we present the modifications required to simulate naval hydrodynamics scenarios, i.e. a moving reference frame, surface piercing body, absorbing beach boundaries; with a demonstration of the hybrid VOF scheme. 


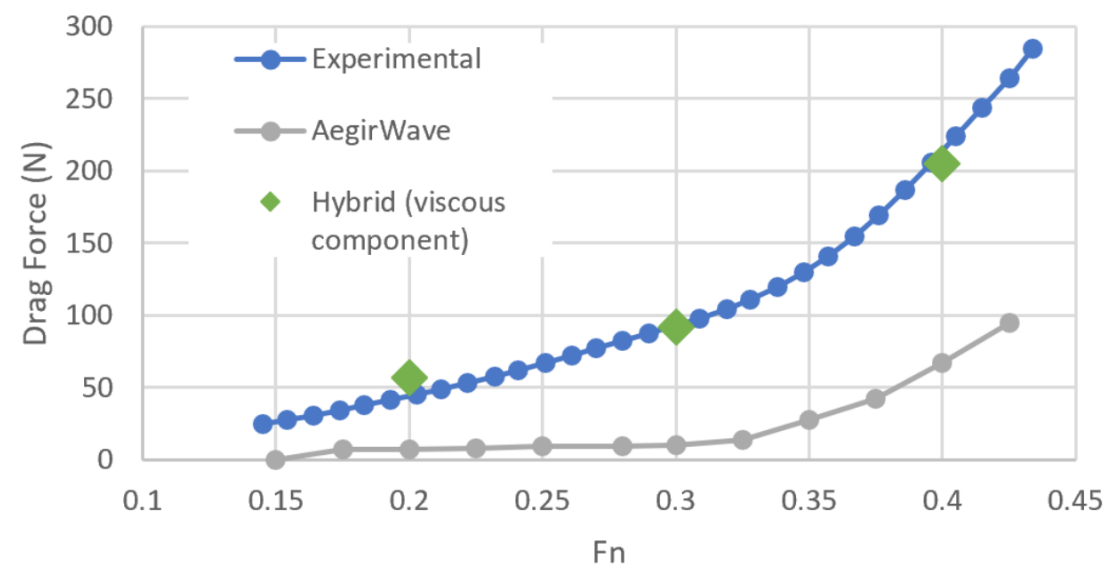

Figure 24: Total steady resistance for the JHSS hull as a function of Froude number. Measurements are shown as blue circles $(\bullet)$, the resistance from wave generation $(\mathrm{BEM})$ is shown as grey circles $(\bullet)$ and the hybrid method solution of wave drag (BEM) and viscous drag (pLBM) with viscous pressure removed is shown as the green diamonds $(\downarrow)$.

\section{Towed hydrofoil}

In this section the towed hydrofoil tests of Duncan (1983) are reproduced with the LBM VOF solver. First a non-breaking wave is simulated in order to validate the VOF approach, then a breaking wave is simulated so that the power dissipation scheme of section 3.5 can be validated. In the experiments, a NACA0012 hydrofoil was towed at a constant speed through a long basin at a fixed orientation and height above the bottom of the basin. The amount of water within the basin was varied so that the foil would see different submergence depths (see Fig. 25) and care was taken to eliminate significant 3D effects during testing. During the experiments, a wave field would be generated that is in steady state relative to the reference frame of the foil for both breaking and non-breaking waves (depending on depth). Measurements of the steady free surface were collected and the amount of additional drag generated by wave breaking was estimated.

The LBM domain setup can be seen in Fig. (25) and the mesh used for these results has an origin at $(x=-1 ., y=0.0, z=0.0)$ meters and extends $(3.0,0.6,0.02)$ meters in the $(x, y, z)$ directions with a grid spacing of $2.0 \times 10^{-3}$ meters. The foil, which was specified using the NACA0012 profile with a chord of 0.203 meters at an angle of attack of $5^{\circ}$ for all runs, is located at $(x=0 ., y=0.175, z=0.0)$ with its center defined at $C / 3(C$ is the foil chord $)$. All simulations were run at a Mach number of $\mathrm{Ma}=0.01$. The simulation is run in the reference frame centered about the moving foil. To avoid nonphysical accelerations, the foil must start from rest, meaning the initial fluid velocity is zero, and only hydrostatic pressure is applied to initialize the PDFs. A ramp up of the foil velocity $u_{i}^{F}$ is then simulated over $T_{r}=2.0$ seconds, using a ramp specified 


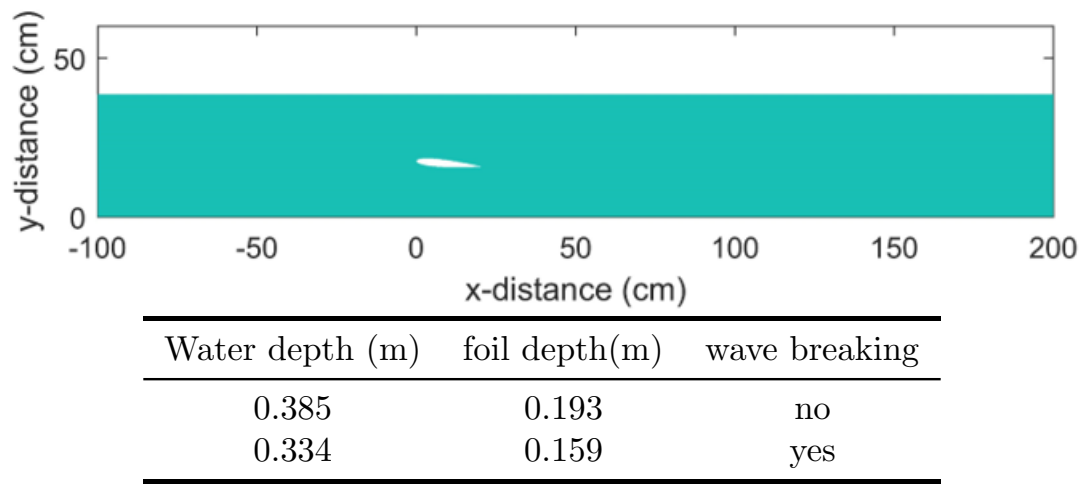

Figure 25: Simulation setup for the towed hydrofoil experiments with a scale visualization the domain (top) where the turquoise region is water and the small white region represents the foil.

by

$$
u_{x}^{F}=\frac{1}{2} \tanh \left(\frac{6 t}{T_{r}}-\frac{1}{2}\right)+\frac{1}{2} \quad \text { and } \quad u_{y}^{F}=u_{z}^{F}=0 .
$$

During the acceleration period a body force is applied to the fluid that is proportional to the acceleration, $F_{1}=\rho \Delta x^{3} \partial u_{1} / \partial t$, during the ramp so that it stays in the correct position relative to the moving frame.

At the inlet boundary $(x=-100 \mathrm{~cm})$ a specified velocity of $u_{i}^{F}$ and hydrostatic pressure are enforced and, to simulate the bottom of the tank in a moving domain, a modified bounce back boundary condition is used (Janssen 2018)

$$
f_{\alpha^{\prime}}=f_{\alpha}-2 \rho_{0} w_{\alpha} \frac{e_{i \alpha} \cdot-u_{i}^{F}}{c^{2}}
$$

for velocities directions reflecting into the domain $\alpha^{\prime}$ and opposite direction $\alpha$. The LBM turbulent wall model was applied as the foil boundary condition and the LES turbulence model was used. Atmospheric pressure is applied at the free surface, a zero horizontal gradient of the DFs at the outlet, and periodic conditions are applied on the sides of the domain (z direction). It was found that an absorbing beach was required to eliminate reflections within the domain and one was set up at $x=160-200$ which followed

$$
\begin{array}{r}
f_{\alpha}^{e q}=(1-R) f_{\alpha}^{e q}\left(\rho^{L B M}, u_{i}^{L B M}\right)+R f_{\alpha}^{e q}\left(\rho^{L B M}, u_{i}^{F}\right) \\
R=1.0-(200-x) / 40
\end{array}
$$

where $\rho^{L B M}$, and $u_{i}^{L B M}$ are calculated from the PDFs at each time, and this modified equilibrium function is used during the collision step.

Fig. (26) shows the simulation results for a tank water depth of 0.385 meters as compared to measurements. Experimental observation showed that a steady state wave field formed behind the 

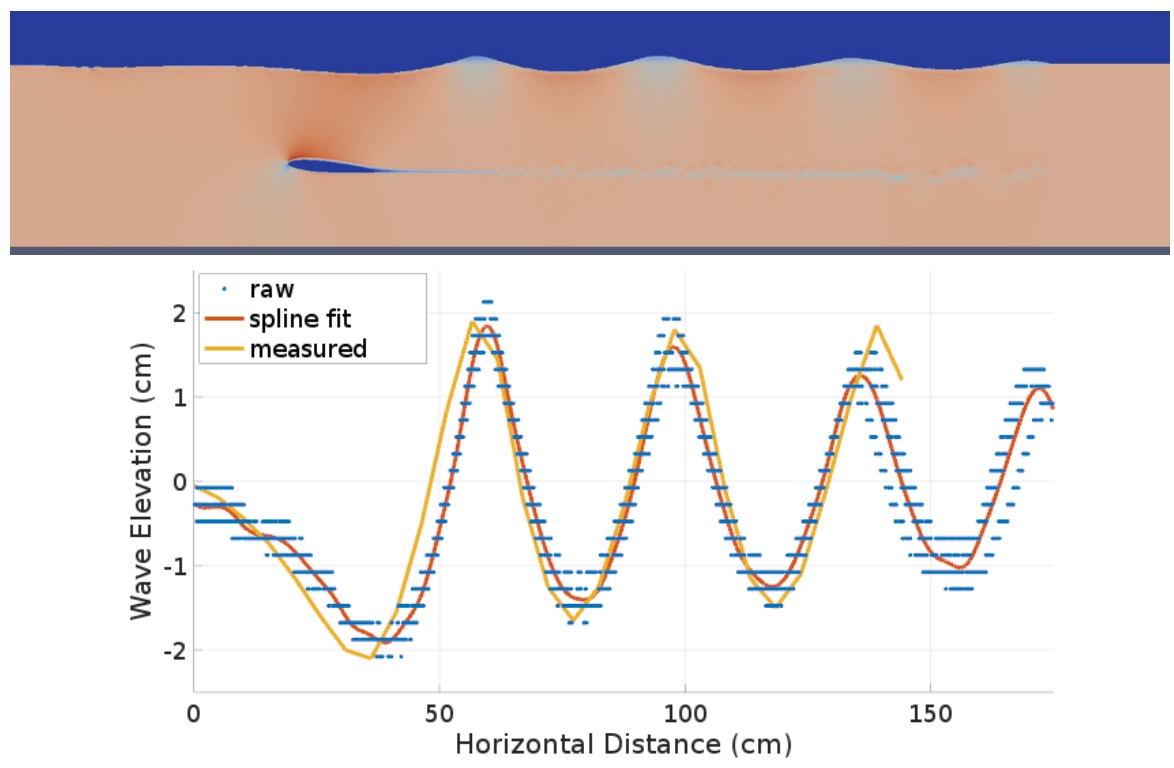

Figure 26: LBM simulation results for the towed hydrofoil and foil submergence depth of 0.193 meters (non-breaking in experiments). (top) Snapshot of the velocity magnitude within the domain. (bottom) Plot of the steady state free surface elevation with measured results $(-)$, nodes containing a volume fraction, $\epsilon, 0.1<\epsilon<0.9(\bullet)$, and a spline fit of those points $(-)$.

foil and no wave breaking was observed. A reasonable agreement in free surface elevation is found when comparing the LBM results to the measurements. Near the center of the measurements (roughly $75 \mathrm{~cm}$ ), a very good agreement is observed and a slightly increasing error is observed as the edges of the measurements are reached. This is possibly a result of lens distortion from the use of a $10 \mathrm{~mm}$ wide angle lens in experimental measurements, which will falsely increase the wave height and wave length that is observed at the edges of the view field, as no image correction is applied in the experiments. The measured results shown here do not have any correction for lens distortion and it is likely that a better agreement would be observed if one were to be applied dependent on the lens characteristics.

Fig. (27) shows the simulation results for a tank water depth of 0.334 meters as compared to measurements. Experimental observation showed that a steady state wave field formed behind the foil and steady state wave breaking was observed at the first crest behind the foil. While a reasonable agreement between the LBM and measurements in free surface elevation was found at some points during the simulation, a steady state breaking wave was not generated. Instead periods of significant wave breaking then wave reforming were observed in the LBM. This discrepancy is the result of the simple VOF scheme used, as a more advanced scheme such as the piecewise linear interface construction (PLIC) would provide better accuracy in calculating the 


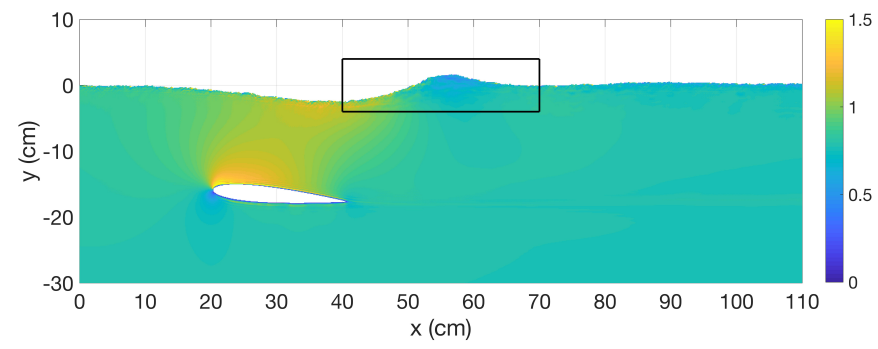

(a)

(b)

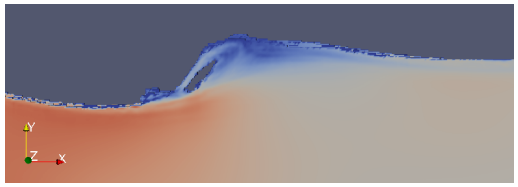

(d)

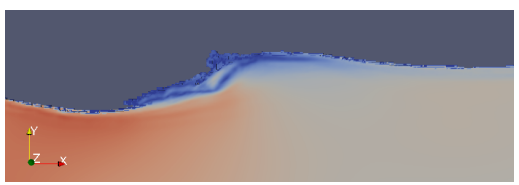

(c)

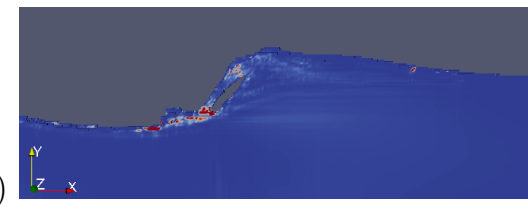

(e)

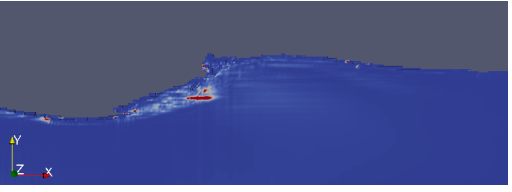

Figure 27: LBM simulation results for the towed hydrofoil and foil submergence depth of 0.159 meters (breaking in experiments). (a) Snapshot of the velocity magnitude within the domain before breaking and the region in black is where the power dissipation will be calculated. Wave breaking visualized within the black region is seen at 3.0 seconds (b and $\mathrm{c}$ ) and 3.5 seconds (d and e). Velocity magnitude is shown (b and d) with blue representing $\left|u_{i}\right|=0.0 \mathrm{~m} / \mathrm{s}$ and red representing $\left|u_{i}\right|=1.5 \mathrm{~m} / \mathrm{s}$. The power dissipation is shown (b and $\mathrm{d}$ ) with blue representing $0.0 \mathrm{~W}$ and red representing representing $1.0 \times 10^{-5} \mathrm{~W}$ calculated within each cell containing a volume of $3.4 \times 10^{-9} \mathrm{~m}^{3}$.

flux of the volume fraction (Eq. 134). Furthermore we have identified that an error exists in the collision step in the LBM with VOF. PDFs streaming from a full cell are given the same treatment as PDFs streaming from a nearly empty fluid cell, likely over representing the momentum of the breaking spray and jet. Although steady breaking was not observed, an initial investigation of the free surface power dissipation scheme was conducted, where Fig. (27a) shows the location where power dissipation is measured and Fig. (27 b-e) show snapshots of the wave breaking with contours of velocity magnitude (b and c) and instantaneous power dissipated (c and e). During this period of time a reasonable agreement between power dissipated is found when comparing the LBM to the hyrdaulic jump originally used in [27]). The hydraulic jump estimate was calculated using a jump of similar height and depth as the breaking wave observed in measurements while the LBM estimate used the total power dissipated within the volume shown. 


\section{Nonlinear wave using the hybrid VOF}

In this section the hybrid VOF scheme is tested by using a highly nonlinear wave as the inviscid solution driving the hybrid VOF. To remove the numerical errors involved with using the BEM to generate the flow, stream function theory is used to generate the FNPF inviscid wave solution [22], so no dissipation from wave breaking is supplied back to the inviscid flow. For increased efficiency, this time and space dependent solution was calculated in parallel on the GPU during simulation. The simulated wave had a height of $H=0.59$ meters, period of $T=1.5$ seconds, and was simulated in a depth of $h=3$ meters which corresponds to a wave length of $L=4.15$ meters. The pLBM domain was 8.3 meters long $(2 L), 4.2$ meters high, and 1 meter wide, using a grid spacing of $2 \mathrm{~cm}$ and a simulation Mach number of Ma $=0.01$. Periodic conditions were applied to the sides of the domain while hydrostatic pressure was enforced at the bottom boundary, $p=p^{I}+p^{P}=p^{H S}$, so with hydrostatic pressure present in the inviscid solution, zero perturbation pressure is applied at the bottom boundary $p^{P}=0$. The simulation was initialized with zero perturbation flow everywhere.

First, the nonlinear wave is simulated alone so that, theoretically, the inviscid flow will provide the total solution. With zero perturbation flow the hVOF scheme should perfectly follow the inviscid solution. However, the inviscid wave was very steep and near the breaking limit so numerical dissipation in the pLBM along with round off errors in the hVOF scheme caused small deviations from the perfectly inviscid solution, providing a test of the total hVOF scheme. The resulting solution, seen in Fig (28), reduced the total wave height slightly relative to the inviscid wave height. The modified DFSBC of Eq. (132) enforces a balance of the driving inviscid flow and dissipation from the perturbation component so that a steady state was reached. During the simulation, a slight increase in total fluid mass of $+0.157 \%$ was observed, which is an acceptable numerical error considering the single precision implementation of the solver.

Next, the same inviscid wave and simulation parameters are simulated except in a wider domain (6 meters instead of 1 meter) and a cylinder is added to the middle of the domain. Typically in the hybrid method the inviscid solution will provide the no-penetration boundary condition to the solid object while the perturbation component will provide the no-slip condition. In this case the stream function inviscid solution cannot consider the cylinder boundary so we apply both cylinder boundary conditions within the pLBM using a zero total velocity condition, 
(a)

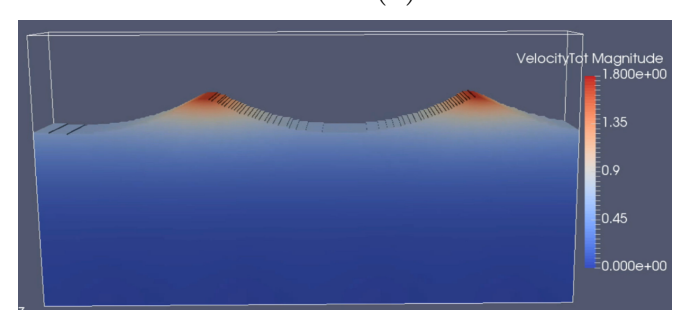

(b)

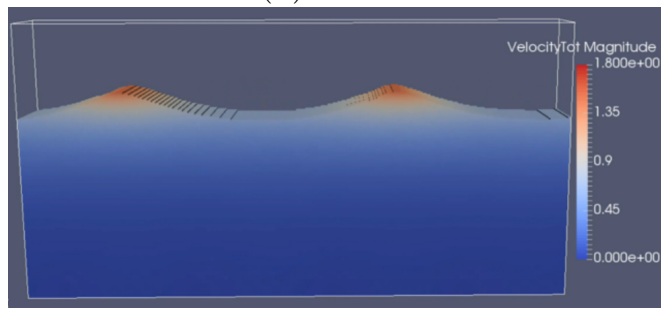

Figure 28: Snapshots of the hVOF simulation of a nonlinear wave using the a fully nonlinear potential flow solution and periodic side boundaries. The solution is shown at simulation start up (a) and at $t=0.9 T$ (b) as a balance between the driving inviscid flow and dissipative effects in the pLBM is reached. Cells shown have a volume fraction of $\epsilon \geq 0.5$ and color contours represent total velocity magnitude $\left(\mathbf{u}=\mathbf{u}_{i}^{I}+\mathbf{u}_{i}^{P}\right)$ with blue representing $|\mathbf{u}|=0.0 \mathrm{~m} / \mathrm{s}$ and red representing $\mathbf{u}=1.8 \mathrm{~m} / \mathrm{s}$.

$\mathbf{u}_{w}^{P}=-\mathbf{u}_{w}^{I}$, which is applied in the pLBM as [37]

$$
f_{\alpha^{\prime}}^{P}\left(\mathbf{x}_{1}, t+\Delta t\right)=f_{\alpha}^{P}\left(\mathbf{x}_{1}, t\right)-2 \rho_{0} w_{\alpha} \frac{\mathbf{e}_{\alpha} \cdot\left(-\mathbf{u}_{w}^{I}\right)}{c^{2}}
$$

in velocity directions $\alpha$, crossing the cylinder boundary, and their opposite counterpart $\alpha^{\prime}$, at lattice nodes of coordinate $\boldsymbol{x}_{1}$ adjacent to the wall.

Simulation results can be seen in Fig. (29), which shows snapshots at approximately quarter period intervals, starting at $t=T / 4$ and ending at $t=T$ (the wave is traveling from left to right). The addition of the cylinder boundary in these steep and nonlinear waves produces a total free surface that is largely perturbed from the inviscid solution, representing a more rigorous test of the hVOF. Regions where $\eta^{T}>\eta^{I}$ can be observed at the front and sides sides of the cylinder, where wave breaking is occurring, and regions where $\eta^{T}<\eta^{I}$ are observed behind the cylinder. For this application, ring waves propagating away from the cylinder should be generated. These are often noticed in potential flow simulations of wave-cylinder interactions, where zero energy dissipation is present. In this simulation ring waves are not observed, likely because of the missing diffracted wave component of the inviscid solution, significant wave breaking that is present, and because any small ring waves that may form would be under-resolved with this pLBM resolution. Finally, small blue streaks (near zero velocity) can be observed on the cylinder in Fig. (29)(b) and (d) which are one cell wide and immediately adjacent to the cylinder boundary. These are a result of the no-slip cylinder boundary condition, while strange in the visualization, are not representative of a problem with the simulation. 
(a)
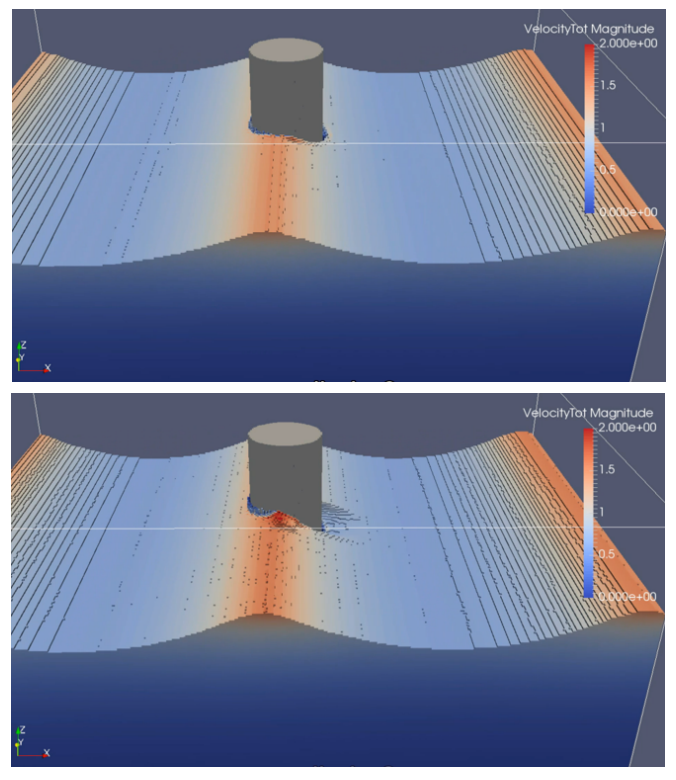

(c) (b)

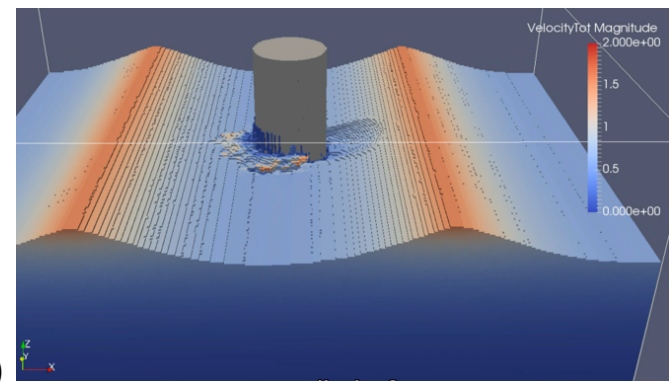

(d)

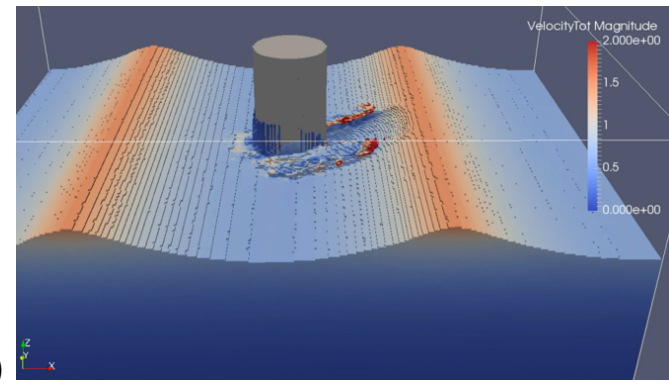

Figure 29: Snapshots of the hVOF simulation of a nonlinear wave interacting with a cylinder (grey) using the a FNPF inviscid wave solution, periodic side boundaries and the cylinder boundary condition is applied in the pLBM. The solution is shown at quarter period $(T)$ intervals starting at time $t=T / 4(\mathrm{a})$ and ending at $t=T(\mathrm{~d})$. Cells shown have a volume fraction of $\epsilon \geq 0.5$ and color contours represent total velocity magnitude $\left(\mathbf{u}=\mathbf{u}_{i}^{I}+\mathbf{u}_{i}^{P}\right)$ with blue representing $|\mathbf{u}|=0.0 \mathrm{~m} / \mathrm{s}$ and red representing $\mathbf{u}=2.0 \mathrm{~m} / \mathrm{s}$.

\subsection{Conclusions}

In this paper we show the progression of the hybrid LBM-BEM solver towards an engineering design tool for the simulation of arbitrary geometries in the presence of a free surface. Previous work validate the method for bodies with an easily defined geometry and analytical solution without the presence of a free surface. While in this work the addition of the gridding tool allows any arbitrary geometry to be simulated without a significant loss of accuracy, provided that the underlying geometry file is of sufficient resolution.

A novel hybrid VOF scheme is presented here and initial validation has been conducted along with a validation of the linear free surface hybrid method. While reasonable validations of these components are shown, either an analytical inviscid solution is used or the inviscid velocity field calculated with the BEM contained significant errors near to the solid body. Further validation will be conducted by coupling the pLBM to a nonlinear free surface BEM solver [48] in future work. 


\subsection{Acknowledgments}

C.M. OReilly, S.T. Grilli, and J.M. Dahl gratefully acknowledge support for this work from grants N000141310687 and N000141612970 of the Office of Naval Research (PM Kelly Cooper). As well as the support of XSEDE grant ENG170010 for GPU computational resources. 


\section{Bibliography}

[1] Abbott, I. H., von Doenhoff A. E. (1959). Theory of wing sections. Dover Publications Inc.

[2] Alessandrini, B. (2007). Thèse d'Habilitation en Vue de Diriger les Recherches. Ecole Centrale de Nantes, Nantes.

[3] Banari A., Janssen C., Grilli S.T. and M. Krafczyk (2014). Efficient GPGPU implementation of a Lattice Boltzmann Model for multiphase flows with high density ratios. Computers and Fluids, 93:1-17.

[4] Banari A., Janssen C.F., and Grilli S.T. (2014). An efficient lattice Boltzmann multiphase model for 3D flows with large density ratios at high Reynolds numbers. Computers and Mathematics with Applications, 68:1819-1843.

[5] Banari A., Mauzole Y., Hara T., Grilli S.T. and C.F. Janssen (2015). The simulation of turbulent particle-laden channel flow by the Lattice Boltzmann method. International Journal for Numerical Methods in Fluids, 79(10):491-513.

[6] Bhatnagar, P. L., Gross, E. P., and Krook, M. (1954). A model for collision processes in gases. i. small amplitude processes in charged and neutral one-component systems. Phys. Rev., 94(3):511-525.

[7] Biausser B., S.T. Grilli, Fraunié P. and Marcer, R. (2004). Numerical analysis of the internal kinematics and dynamics of three-dimensional breaking waves on slopes. International Journal of Offshore and Polar Engineering, 14(4):247-256.

[8] Bouzidi, M., Firdaouss, M., and Lallemand, P. (2001). Momentum transfer of a LatticeBoltzmann fluid with boundaries. Physics of Fluids, 13:3452-3459.

[9] Brent, R. P. (1973). Algorithms for Minimization Without Derivatives. Prentice Hall. Reviewed in: American Scientist 61 (May-June 1973), 374; Mathematical Programming 4 (1973), 349; Computer J. 16 (1973), 314; Math. Comp. 28 (1974), 865-866.

[10] Cusanelli, D.S. (2007). Joint High Speed Sealift (JHSS) Baseline Shaft and Strut (Model 5653) Series 1: Bare Hull Resistance, Appended Resistance, and Alternative Bow Evalua- 
tions. Naval Surface Warfare Center Hydromechanics Department Report, NSWCCD-50-TR20071066 .

[11] Dean, R. G. and Dalrymple, R. A. (1991). Water wave mechanics for engineers and scientists (Vol. 2). World Scientific Publishing Company.

[12] d'Humieres, D., Ginzburg, I., Krafczyk, M., Lallemand, P., and Luo, L.-S. (2002). Multiple relaxation-time Lattice Boltzmann models in three-dimensions. Royal Society of London Philosophical Transactions Series A, 360:437-451.

[13] Filippova, O., Hänel, D. (2008). Boundary-fitting and local grid refinement for lattice-BGK models. International Journal of Modern Physics, C 9 (1998) 12711279.

[14] Freudiger, S., Hegewald, J., and Krafczyk, M. (2008). A parallelization concept for a multiphysics lattice Boltzmann solver based on hierarchical grids. Progress in Computational Fluid Dynamics, 8(1-4):168-178.

[15] Frisch, U., d'Humières, D., Hasslacher, B., Lallemand, P., Pomeau, Y., and Rivet, J.-P. (1987). Lattice Gas Hydrodynamics in Two and Three Dimensions. J. Complex Syst., 1:75136.

[16] Geller, S., Krafczyk, M., Tölke, J., Turek, S., and Hron, J. (2006). Benchmark computations based on Lattice-Boltzmann, Finite Element and Finite volume Methods for laminar Flows. Comp. and Fluids, 35:888-897.

[17] Ginzburg, I. and D'Humieres, D. (2003). Multi-reflection boundary conditions for lattice Boltzmann models. Physical Review E, 68(6):066614.1-066614.30.

[18] Grilli, S., Jorrillo, J., (1997) Numerical Generation and Absorption of Fully Nonlinear Periodic Waves. Journal of Engineering Mechanics 1060-1069 October 1997.

[19] Grilli, S.T. (2008) On the development and application of hybrid numerical models in nonlinear free surface hydrodynamics. Keynote lecture in Proc. 8th Intl. Conf. on Hydrodynamics (Nantes, France, 9/08) (P. Ferrant and X.B. Chen, eds.), pps. 21-50.

[20] Grilli, S.T., Dias, F., Guyenne, P., Fochesato, C. and F. Enet (2010). Progress in fully nonlinear potential flow modeling of 3D extreme ocean waves. Chapter 3 in Advances in Numerical Simulation of Nonlinear Water Waves (ISBN: 978-981-283-649-6, edited by Q.W. Ma) (Vol. 
11 in Series in Advances in Coastal and Ocean Engineering). World Scientific Publishing Co. Pte. Ltd., pps. 75-128.

[21] Grilli, S., Guyenne, P., and Dias, F. (2001). A fully nonlinear model for three-dimensional overturning waves over arbitrary bottom. International Journal for Numerical Methods in Fluids, 35(7):829-867.

[22] Grilli, S.T. and Horrillo, J. (1997). Numerical Generation and Absorption of Fully Nonlinear Periodic Waves. Journal of Engineering Mechanics, 123(10):1,060-1,069.

[23] Grilli, S.T. and Subramanya, R. (1996). Numerical Modeling of Wave Breaking Induced by Fixed or Moving Boundaries. Computational Mechanics, 17(6):374-391.

[24] Grilli, S.T., Svendsen, I.A. and Subramanya, R. (1997). Breaking criterion and characteristics for solitary waves on slopes. Journal of Waterway Port Coastal and Ocean Engineering, 123(3):102-112.

[25] Gueyffier, D., Li, J., Nadim, A., Scardovelli, R., and Zaleski, S. (1999). Volume-of-fluid interface tracking with smoothed surface stress methods for three-dimensional flows. Journal of Computational Physics, 152(2):423-456.

[26] Guignard, S., Grilli, S.T., Marcer, R. and Rey, V. (1999). Computation of shoaling and breaking waves in nearshore areas by the coupling of BEM and VOF methods. In Proc. 9th Offshore and Polar Engng. Conf. (ISOPE99, Brest, France, May 1999), Vol. III, 304-309.

[27] Guignard, S. and S.T., Grilli. (2001). Modeling of shoaling and breaking waves in a 2D-NWT by using a spilling breaker model. Proc. 11th Offshore and Polar Engng. Conf. (ISOPE01, Stavanger, Norway, June 2001), Vol III,116-123.

[28] Guo, Z., Zheng, C., and Shi, B. (2002). Discrete lattice effects on the forcing term in the Lattice Boltzmann method. Physical Review E, 65(4):046308.1-046308.6.

[29] Harris, J. and Grilli, S.T. (2010). A perturbation approach to large-eddy simulation of wave-induced bottom boundary layer flows. Intl. J. Numer. Meth. Fluids, 68, 1,574-1,604

[30] He, X. and Luo, L.-S. (1997). Lattice Boltzmann model for the incompressible Navier-Stokes equation. Journal of Statistical Physics, 88:927-944. 
[31] Hirt, C. and Nichols, B. (1981). Volume of fluid method for dynamics of free boundaries. Journal of Computational Physics, 39:201-221.

[32] Janssen, C. and Krafczyk, M. (2010). A lattice Boltzmann approach for free-surface-flow simulations on non-uniform block-structured grids. Computers and Mathematics with Applications, 59 (7):2215-2235.

[33] Janssen, C.F. (2010) Kinetic approaches for the simulation of non-linear free surface flow problems in civil and environmental engng. $\mathrm{PhD}$ thesis, Technical Univ. Braunschweig.

[34] Janssen C.F., S.T. Grilli and M. Krafczyk (2010) Modeling of Wave Breaking and WaveStructure Interactions by Coupling of Fully Nonlinear Potential Flow and Lattice-Boltzmann Models. Proc. 20th Offshore and Polar Engng. Conf. (ISOPE10, Beijing, China, June 20-25, 2010), pps. 686-693. Intl. Society of Offshore and Polar Engng.

[35] Janssen, C.F., S.T. Grilli and M. Krafczyk (2013). On enhanced non-linear free surface flow simulations with a hybrid LBM-VOF approach. Computers and Mathematics with Applications, $65(2): 211-229$

[36] Janssen, C.F., S.T. Grilli and M. Krafczyk (2013). On enhanced non-linear free surface flow simulations with a hybrid LBM-VOF approach. Computers and Mathematics with Applications, 65(2), 211-229, doi:10.1016/j.camwa.2012.05.012

[37] Janssen, C.F., O’Reilly, C.M., S.T. Grilli (2018). A lattice-Boltzmann-based perturbation method. To be submitted to the Journal of Computers and Fluids

[38] Junk, M., Klar, A., and Luo, L.-S. (2005). Asymptotic analysis of the lattice Boltzmann equation. Journal of Computational Physics, 210(2):676-704.

[39] Körner, C., Thies, M., Hofmann, T., Thürey, N., and Rüde, U. (2005). Lattice Boltzmann model for free surface flow for modeling foaming. Journal of Statistical Physics, 121(1-2):179196(18).

[40] Krafczyk, M., Tölke, J., and Luo, L.-S. (2003). Large-eddy simulations with a multiplerelaxation-time LBE model. Int. J. Mod. Phys. B, 17:33-39.

[41] Lallemand, P. and Luo, L.-S. (2000). Theory of the lattice Boltzmann method: Dispersion, dissipation, isotropy, galilean invariance, and stability. Physical Review, E 61:6546-6562. 
[42] Latt, J. (2007). Hydrodynamic limit of lattice Boltzmann equations. PhD thesis, Univ. Genéve

[43] Li, Y., Zhang, R., Shock, R., and Chrn, H. (2009). Prediction of vortex shedding from a circular cylinder using a volumetric Lattice-Boltzmann boundary approach. The European Physical Journal Special Topics, 171, 91-97.

[44] Longuet-Higgins, M.S. (1953). Mass transport in water waves. Phil. Trans. Royal Soc. London, A, 535-581.

[45] Martin, J. and Moyce, W. (1952). An experimental study of the collapse of liquid columns on a rigid horizontal plane. Technical report.

[46] Mei, R., Luo, L.-S., Shyy, W. (1999). An Accurate Curved Boundary Treatment in the Lattice Boltzmann Method. Journal of Computational Physics, 155(2):307-330.

[47] Mei, R., Luo, L.-S., Lallemand, P., and d'Humieres, D. (2006). Consistent initial conditions for lattice boltzmann simulations. Computers and Fluids, 35(8-9):855-862.

[48] Mivehchi A., Harris, J.C., Grilli, S.T., Dahl, J.M., O’Reilly, C.M., Kuznetsov, K. and Janssen, C.F. (2017). A hybrid solver based on efficient BEM-potential and LBM-NS models: recent BEM developments and applications to naval hydrodynamics. Proc. 27th Offshore and Polar Engng. Conf.(ISOPE17, San Francsico, USA. June 2017), Intl. Society of Offshore and Polar Engng., pps. 721-728

[49] Möller, Tomas; Trumbore, Ben (1997). Fast, Minimum Storage Ray-Triangle Intersection. Journal of Graphics Tools

[50] O'Reilly, C., Grilli, S.T., Dahl, J.M., Banari, A., Janssen, C.F., Shock, J.J. and M. Uberrueck. (2015) Solution of viscous flows in a hybrid naval hydrodynamic scheme based on an efficient Lattice Boltzmann Method. Proc. 13th Intl. Conf. on Fast Sea Transportation (FAST 2015; Washington D.C., September 1-4, 2015), 9 pps., https://personal.egr.uri.edu/grilli/OReilly-etal-FAST2015.pdf.

[51] O’Reilly C.M., Grilli S.T., Harris J.C., Mivehchi A., Janssen C.F. and J. Dahl (2016). Development of a hybrid LBM-potential flow model for Naval Hydrodynamics. Proc. 15th Journée de l'hydrodynamique (JH2016) (November 22-24, Brest, France), 15 pps., http://website.ecnantes.fr/actesjh/images/15JH/Articles/oreilly.pdf. 
[52] O'Reilly C.M., S.T. Grilli, J.C. Harris, A. Mivehchi, C.F. Janssen and J.M. Dahl (2017). A Hybrid Solver Based on Efficient BEM-potential and LBM-NS Models: Recent LBM Developments and Applications to Naval Hydrodynamics. In Proc. 27th Offshore and Polar Engng. Conf. (ISOPE17, San Francsico, USA. June 2017), Intl. Society of Offshore and Polar Engng., pps. 713-720, https://personal.egr.uri.edu/grilli/OReillyetal_ISOPE17.pdf.

[53] O’Reilly C.M., S.T. Grilli, C.F. Janssen, J.M. Dahl, A. Mivehchi and J.C. Harris (2018). A hybrid lattice Boltzmann method and potential flow solver as applied to high Reynolds number, incompressible fluid flows. To be submitted to the Journal of Computational Physics

[54] Parker, B. and Youngs, D. (1992). Two and three dimensional eulerian simulation of fluid flow with material interfaces. Technical report, UK Atomic Weapons Establishment.

[55] Quian, Y. H., d'Humieres, D., and Lallemand, P. (1992). Lattice BGK models for Navier Stokes equations. Europhysics Letters, 17:479-484.

[56] Rajani, B., Kandasamy, A., and Majumdar, S. (2009). Numerical simulation of laminar flow past a circular cylinder. Applied Mathematical Modelling, 33, 1228-1247.

[57] Reliquet, G., A. Drouet, P.-E. Guillerm, L. Gentaz and P. Ferrant (2014). Simulation of wave-ship in ction in regular and irregular seas under viscous flow theory using the SWENSE method. Proc. 30th Symp. Naval Hydrod., (Tasmania, 11/2014), 11 pps.

[58] Salih, A. and Moulic, S. G. (2006). A level set formulation for the numerical simulation of impact of surge fronts. SADHANA - Academy Proceedings in Engineering Sciences, 31:697707.

[59] Stålberg, E., Bruger, A., Lotstedt, P., Johansson, A., and Henningson, D. (2006). High Order Accurate Solution of Flow Past a Circular Cylinder. Journal of Scientific Computing, Vol 27 .

[60] Stokes G.G. (1851). On the effect of the internal friction of fluids on the motion of pendulums. Cambridge Philosophical Transactions; 9:8-106.

[61] Tölke, J.(2008). Implementation of a lattice Boltzmann kernel using the compute unified device architecture developed by NVIDIA. Computing and Visualization in Science, 1:29-39.

[62] Tölke, J. and Krafczyk, M. (2008b). Teraflop computing on a desktop PC with GPUS for 3D CFD. International Journal of Computational Fluid Dynamics, 22:443-456. 
[63] Wieselsberger, C. (1932). New data on the laws of fluid resistance. Technical Notes. National Advisory Committee for Aeronautics, No. 84.

[64] Youngs, D. (1982). Time-dependent multimaterial flow with large fluid distortion. In Morton, K. and Baines, M., editors, Numerical Methods for Fluid Dynamics, pages 273-285. Academic Press. 